\title{
Engineering Plasmonic Nanostructures for Light Management and Sensing
}

sujan phani kumar kasani

West Virginia University, sukasani@mix.wvu.edu

Follow this and additional works at: https://researchrepository.wvu.edu/etd

Part of the Biomedical Commons, Electromagnetics and Photonics Commons, Electronic Devices and Semiconductor Manufacturing Commons, Nanoscience and Nanotechnology Commons, and the Semiconductor and Optical Materials Commons

\section{Recommended Citation}

kasani, sujan phani kumar, "Engineering Plasmonic Nanostructures for Light Management and Sensing" (2019). Graduate Theses, Dissertations, and Problem Reports. 7481.

https://researchrepository.wvu.edu/etd/7481

This Dissertation is protected by copyright and/or related rights. It has been brought to you by the The Research Repository @ WVU with permission from the rights-holder(s). You are free to use this Dissertation in any way that is permitted by the copyright and related rights legislation that applies to your use. For other uses you must obtain permission from the rights-holder(s) directly, unless additional rights are indicated by a Creative Commons license in the record and/ or on the work itself. This Dissertation has been accepted for inclusion in WVU Graduate Theses, Dissertations, and Problem Reports collection by an authorized administrator of The Research Repository @ WVU.

For more information, please contact researchrepository@mail.wvu.edu. 


\title{
Engineering Plasmonic Nanostructures for Light Management and Sensing
}

\author{
Sujan Kasani
}

\author{
Dissertation submitted \\ to Benjamin M. Statler College of Engineering and Mineral Resources \\ at West Virginia University \\ in partial fulfillment of the requirements for the degree of \\ Doctor of Philosophy in \\ Electrical Engineering
}

Nianqiang Wu, Ph.D., Co-Chair, research advisor.

Yuxin Liu, Ph.D., Chair, academic advisor.

Lawrence A. Hornak, Ph.D.

Dimitris Korakakis, Ph.D.

Terence Musho, Ph.D.

Lane Department of Computer Science and Electrical Engineering Morgantown, West Virginia, United States

2019

Keywords: Localized Surface Plasmon Resonance, Biosensor, Nano Sphere Lithography, Metallic Nanostructures, Surface-Enhanced Raman Scattering, Plasmon-Enhanced Fluorescence, Nanoring array, Nanopyramid Array, Finite-Difference Time-Domain. 


\section{Abstract Engineering Plasmonic Nanostructures for Light Management and Sensing}

\section{Sujan Kasani}

The two major global problems are to provide health safety and to meet energy demands for ever growing population on a large scale. The study of light interaction with nanostructures has shown a promising solution in improving the fields of bio-sensor and solar energy devices which addresses above mentioned two major global problems. Nanostructures have tunable physicochemical properties such as light absorption, electrical and thermal properties unlike bulk materials, which gives an advantage in applications like bio-sensing and energy harvesting devices. The development of nanofabrication techniques along with the discovery of Surface Enhanced Raman Scattering (SERS) and Plasmon Enhanced Fluorescence (PEF), led to the development of Point of Care (POC) sensing devices. The fundamental understanding of light path in a nanostructured material led to the improvement in solar energy harvesting performance. For both of these applications, engineering nanostructures is the key to improving performance.

In this work, different plasmonic nanostructures were designed, fabricated and analyzed for biosensor and light management applications. A new fabrication route, which combines nanosphere lithography with silicon-based clean-room microfabrication processes, has been developed to produce large-area long-range ordered gold nanoring array patterns in a controllable fashion. The developed nanoring structure has SERS enhancement of $2 * 10^{9}$ and is used for miRNA detection. A novel pyramid array on gold film 3D plasmonic nanostructure is designed to convert plasmonic light scattering to confined light absorption. This structure generates a cavity mode by hybridization of fundamental modes, which creates a strong electric and magnetic field with a large mode volume. Due to its unique properties pyramids coupled film structure is used for both solar light management device and in Metal Enhanced Fluorescence (MEF). The fabricated structure is used to demonstrate plexiton (plasmon - exciton coupling) generation and is very effective in light trapping in the gap mode. In MEF, the sandwich nanostructure is used for Metal Organic Framework (MOF) fluorescence enhancement and the enhancement factor is around $5 * 10^{2}$. 
With the plasmonic metal nanostructure optimization, the performance of a specific application is improved. However, the metals used for plasmonic applications are noble metals like gold and silver to support strong localized surface plasmon resonance (LSPR), which are expensive. Two-dimensional semiconductor materials have shown plasmon resonance in the visible region, having a lot of applications in sensing and photonics. Heavily doped semiconductors could replace expensive metals without compromising the performance. LSPR in metals is tuned by shape, size and refractive index of surroundings. This restricts plasmon resonance tuning over a narrow wavelength range and need to choose a different metal to exceed the rage of application. In contrast, LSPR in plasmonic semiconductors can be tuned with parameters like carrier density, annealing temperature and doping. This gives an advantage of tuning the plasmon peak over a broad range including visible, Near Infrared (NIR) and Infrared(IR) regions. This is because, for semiconductor materials, the carrier concentration can be varied over a large range. Herein, the molybdenum oxide thin films were directly deposited and nitrogen annealed which showed a tunable localized surface plasmon resonance (LSPR). A chip based 2D semiconductor material is fabricated to study the structural and size dependent plasmon resonance. This work establishes a way to fabricate chip based ordered semiconductor nanostructures, which helps in a systematic study of plasmon properties on nanostructures. 
"I can only give you wings to fly; it is your duty to reach the highest point"

- Mr. K.V. Ramana Rao, my father and teacher. 


\section{Acknowledgements}

I would like to express my deepest gratitude to my research advisor Dr. Nianqiang $\mathrm{Wu}$, for his invaluable assistance, support and encouragement throughout my research. This dissertation would not have been possible without his intellectual support and the resources he made available to me. Besides my advisor, I would like to express my sincere thanks to my $\mathrm{PhD}$ dissertation committee chair, Dr. Yuxin Liu.

I am thankful to my committee members Dr. Lawrence Hornak, Dr. Dimitris Korakakis and Dr. Terence Musho for their encouragement, technical discussions and feedback.

I sincerely acknowledge Harley Hart, Dr. Kolin Brown, Dr. Qiang Wang and Dr. Marcela Redigola for their support in using WVU Shared Research Facilities.

Immense thanks to my labmates Dr. Peng Zheng, Dr. Xuefei Gao, Savan Suri, Kathrine Curtin, Dr. Scott Cushing, Dr. Hui Yang, Joseph Bright, Botong Liu, Prof. Haibin Tang, Jennifer Boryczka, Prof. Anveeksh Koneru, Shimeng Hao and Yang He for their help and knowledge exchange.

Last but not the least, I owe my sincere thanks and dedicate this dissertation to my father Ramana Rao, mother Padhma Latha, my sister Pavani and my friends whose love and sacrifice for me is beyond anything I will ever understand.

Sujan Kasani

West Virginia University

July 2019 


\section{Contents}

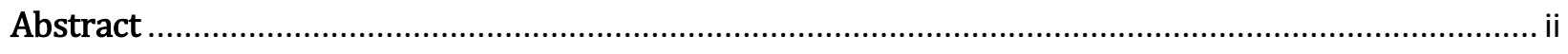

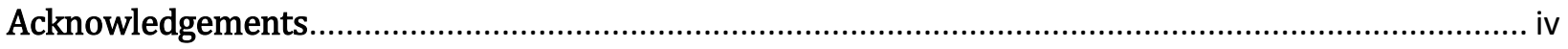

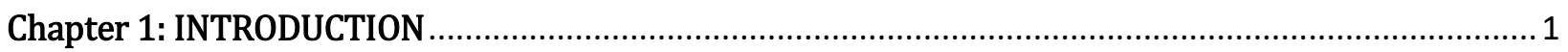

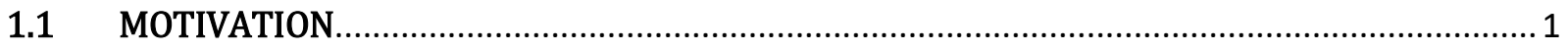

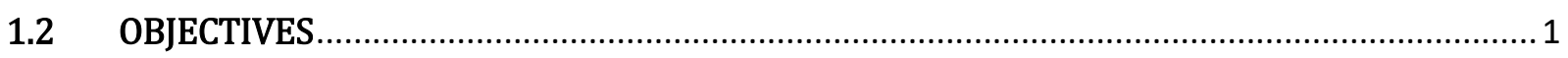

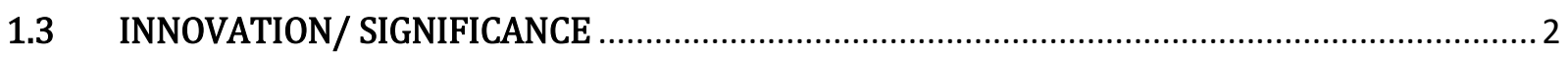

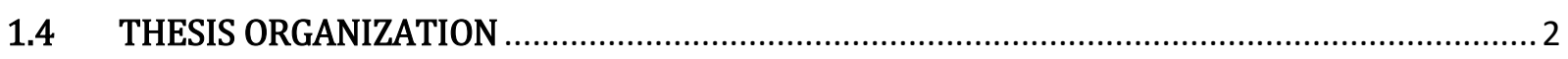

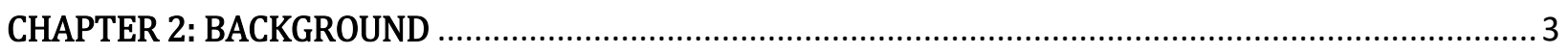

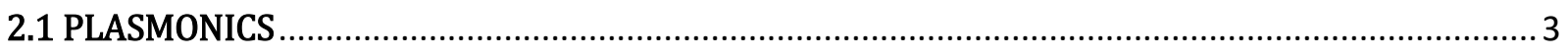

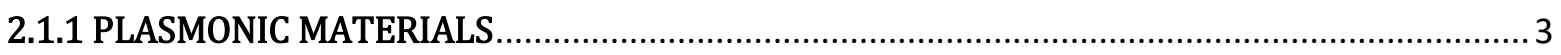

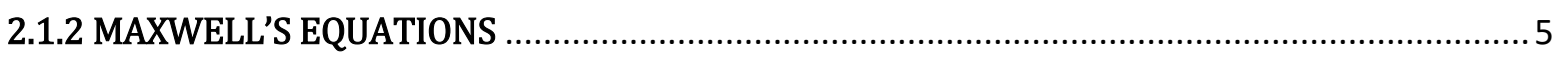

2.2 IMPORTANCE OF NANOSTRUCTURE ARRAY FOR PLASMONIC APPLICATIONS ........................... 6

2.3 FABRICATION TECHNIQUES FOR NANOSTRUCTURE ARRAY ………………………….............

2.4 PLASMON MODES in 2D AND 3D NANOSTRUCTURES …………………………………....12

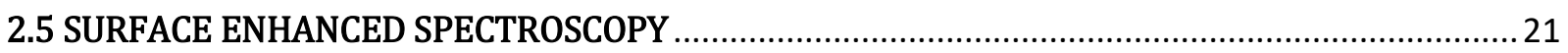

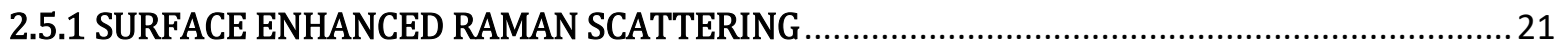

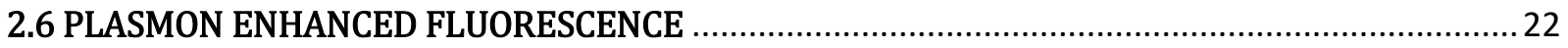

2.7 LIGHT MANAGEMENT APPLICATIOS USING NANOSTRUCTURES ………………………........24

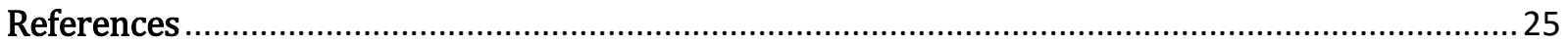

CHAPTER 3: FABRICATION AND OPTICAL PROPERTIES OF LARGE-AREA PLASMONIC GOLD NANO

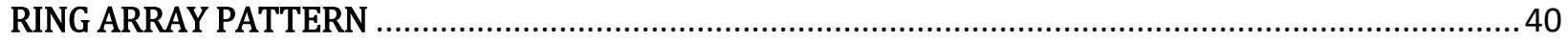

3.1 INTRODUCTION

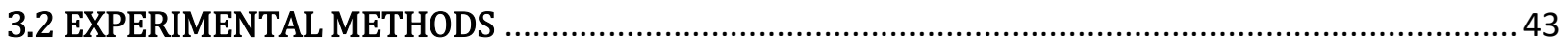

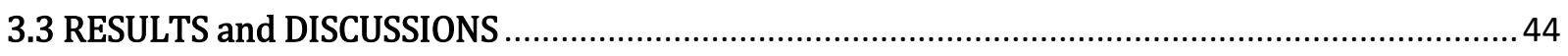

3.4 CONCLUSIONS

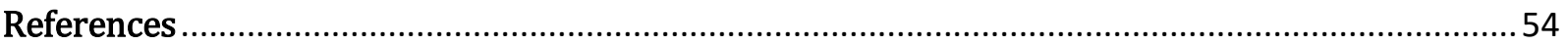

CHAPTER 4: LIGHT MANAGEMENT AND PLEXITON CREATION BY PLASMONIC GAP MODE …..........57

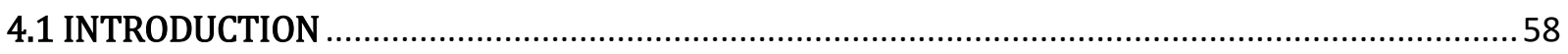

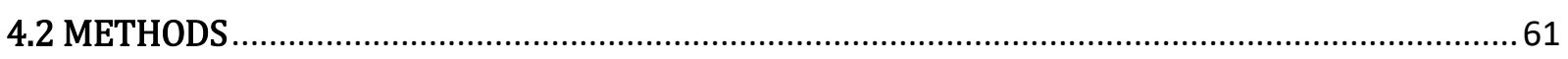

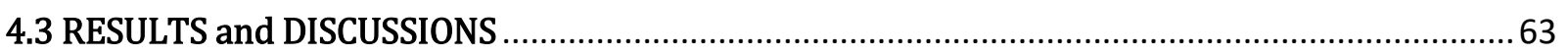

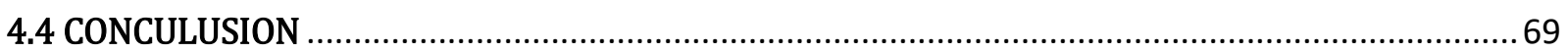

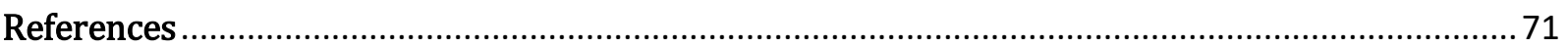


CHAPTER 5: ENHANCED FLUORESCENCE OF METAL ORGANIC FRAMEWORKS IN PLASMONIC GAP

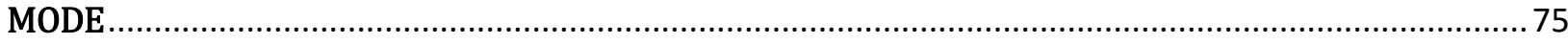

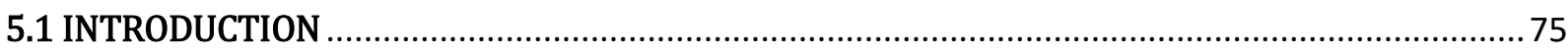

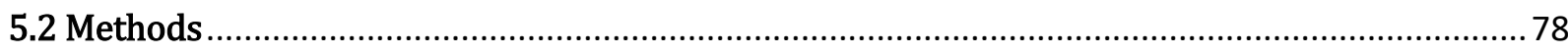

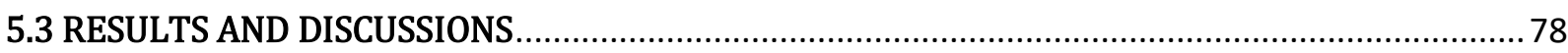

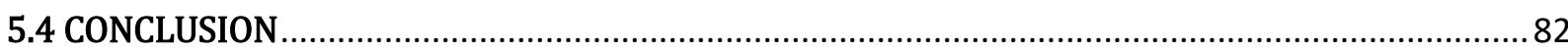

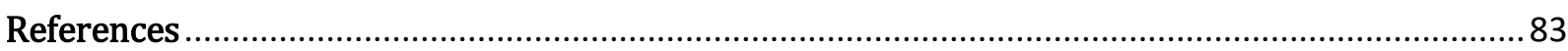

CHAPTER 6: INEXPENSIVE SEMICONDUCTOR MATERIALS FOR PLASMONIC APPLICATIONS ............85

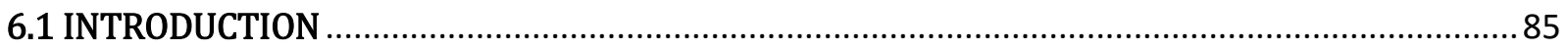

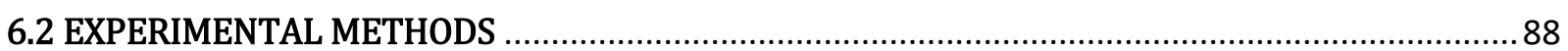

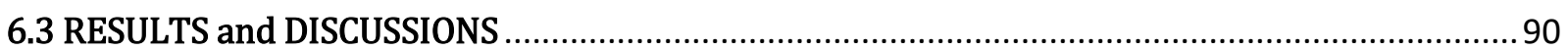

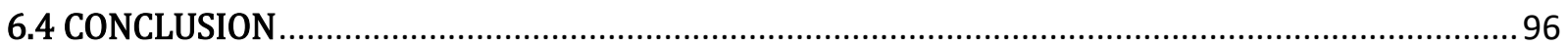

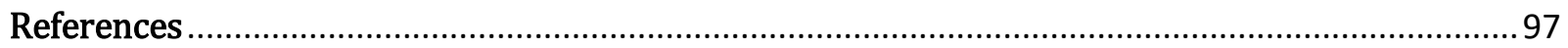

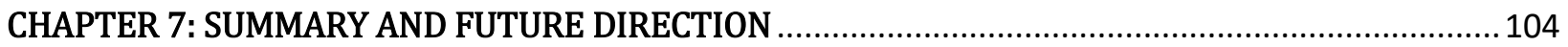

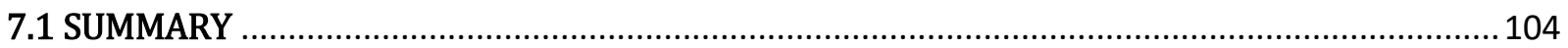

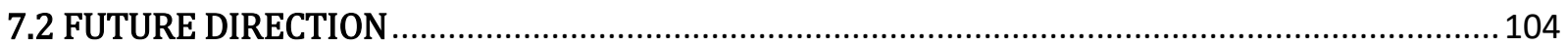

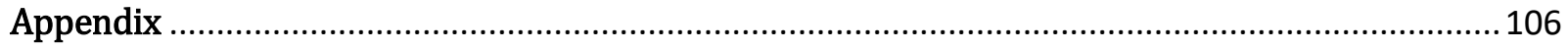




\section{Chapter 1: INTRODUCTION}

\subsection{MOTIVATION}

With huge population growth, proper health care and increase in energy requirements are inevitable. In the last two decades, there was tremendous growth in medical diagnosis however most of the tools for disease and bio-molecule detection are expensive and time taking. There is a demand for economical technologies for early detection of disease to improve health care. An accurate device which can detect the health condition and environmental pollutants economically and portable can contribute significantly to the development of health care and environmental safety.

Energy requirements are increasing day by day with the ever growing population. There is a need to move from a traditional non-renewable source of energy to a clean energy source like solar energy. In this aspect, light management is very crucial to improve the performance of optoelectronic devices like photodetectors, solar cells and LEDs. Therefore, these two main global problems which are health care and energy requirements should be addressed with effective solutions.

\subsection{OBJECTIVES}

This thesis explains the science of light interaction with nanostructures. Specifically, how to engineer plasmonic nanostructures for biosensor and light management applications. The main concepts covered in the dissertation are fabrication of highly ordered nanostructure arrays for SERS/PEF based biomolecule detection, studying generated plasmon modes, optical path and light management in nanostructure arrays. The objectives are listed below

1. To develop large scale nanofabrication by implementing Nanosphere lithography (NSL) technique to fabricate periodic plasmonic array pattern in a controlled fashion.

2. To study the evolution of the plasmonic and optical properties of nanostructure which correlates with SERS/PEF performance. 
3. To study the plasmon hybridized mode to analyze the light path in the nanostructure for light management applications.

4. To study new plasmonic materials that address limitations of cost, losses and stability.

\subsection{INNOVATION/ SIGNIFICANCE}

The existing fabrication technologies and nanostructure design such as free standing nanoparticles usage for sensing and solar applications are not reliable. Free standing nanoparticles aggregate in a solution altering the plasmon mode which in turn affects the performance of the device. Also, the particles used are expensive noble metals. These problems prompt exploration of an alternative innovative solution. The proposed design and fabrication of nanostructures addressed the problems of the existing methods. The proposed research is significant in

1. Developing highly repeatable, uniform and large scale nanostructures.

2. Innovates sensing mechanism by optimizing the structure based on new plasmon coupling modes analysis.

3. Exploring the possibility of replacing existing expensive material.

\subsection{THESIS ORGANIZATION}

The document encompasses seven chapters. Chapters 2,3,4,5 and 6 are expanded versions of published, submitted or soon to be submitted manuscripts. The first chapter details the introduction and objective of the thesis. The second chapter discusses the theoretical background and physics of plasmonics along with the fabrication techniques and applications. The third chapter focuses on the study of miRNA detection biosensor using gold nanoring array. In chapter four, the coupling mechanism of two different nanostructures for plexiton generation was discussed. In chapter five, the plasmonic properties of a semiconductor material and their impact on applications were discussed. In chapter six, the fluorescence sensing mechanism using plasmonic gap plasmon mode was presented in detail. 


\section{CHAPTER 2: BACKGROUND}

\subsection{PLASMONICS}

Plasmonics is the study of the interaction between the electromagnetic field and free electrons in the metal. Free electrons in the metal can be excited by the electric component of light and create collective oscillations. These oscillations displace the free electrons from their corresponding nuclei. This displacement causes a dipole with the incident light ${ }^{1}$. At a particular frequency, the frequency of oscillation of dipole match with the frequency of incident light, which is called plasmon resonance. The incident light couples with the plasmons which either strongly scatter or absorb light. Plasmon and light interaction allows breaking the diffraction limit of light. This causes localization of light in subwavelength dimensions. This localization of light in subwavelength dimensions' produces enhanced optical near-field and strong electromagnetic (EM) field enhancement. If the localization of light takes place at the metal-dielectric interface as propagating waves, the excitation is known as surface plasmon polaritons (SPP), while if it takes place within the nanostructure, localized surface plasmon resonance (LSPR) is excited ${ }^{2}$. Plasmonic behavior is seen in noble metals like gold, silver and copper. The localized plasmon resonance phenomenon occurs when the nanostructure size is comparable or less than the wavelength of light. The plasmon resonance properties mainly depend on the nanostructure shape, size and the dielectric medium in which the nanostructure is present. The attractive phenomenon of SPR and LSPR enables plasmonic materials with high potential of applications in a variety of fields which include photonics ${ }^{3}, \operatorname{SERS}^{4,5}$, sensors ${ }^{6,7}$ energy $^{8}$ and light management ${ }^{9}$.

\subsubsection{PLASMONIC MATERIALS}

There are many materials that show plasmonic behavior which include metals, metalalloys, and semiconductors. The dielectric function of the material is key in determining where and how intense the plasmon resonance. The real part of the material dielectric function needs to be negative which is satisfied if the plasma frequency of the material is higher than the frequency of interest. Furthermore, the imaginary part of the materials dielectric function has to be low as 
it corresponds to the losses experienced when polarizing the material. Each material has its advantages and disadvantages making the right material based on wavelength region, stability and refractive index.

The plasma frequency of a material is given by $\omega_{p}=\sqrt{\frac{n_{e}^{2}}{m_{e} \epsilon_{0}}}$, where $\mathrm{n}_{\mathrm{e}}$ is the density of electrons and $m_{\mathrm{e}}$ is the effective mass of electrons. ${ }^{10}$

The most typical materials used for plasmonic application are the ones with high plasma frequency which allows plasmon resonance to fall in the visible region of spectra. This is because most of the optoelectronic and solar applications are performed in visible region.

The other important parameter is conductivity ${ }^{11}$.

$$
\gamma=\frac{N e^{2}}{\sigma m_{e}}
$$

where $\sigma$ is the conductivity and $\gamma$ is damping constant.

Conductivity is inversely proportional to damping constant. Thus materials having high conductivity, in particular, gold and silver have intense plasmon resonance. Both materials have very low losses in the visible and near infrared (NIR) region often making them the optimal choice. The plasmon resonance is strong for highly conductive materials and collapses for low conductive or insulating materials Silver having the lowest loss of all metals often seems the best choice but silver is also very easily oxidized in the presence of oxygen.

Many metallic alloys are not of interest as they do not give any advantage or have too many disadvantages compared to pure metals.

Recently, semiconductor nanocrystals (NCs) like metal chalcogenides, metal oxides, metal nitrides and heavily doped semiconductors were investigated for plasmonics ${ }^{12,13}$.

Semiconductors can be used as plasmonic material if the plasma frequency is higher than the region of interest and the bandgap is high enough to ensure minimal losses due to interband transitions. Furthermore, to reach lower frequencies for phonon resonances to occur, the semiconductor material needs to be heavily doped while still ensuring high carrier mobility. Due to that, only a limited number of materials useable for plasmonics has until now been found despite a large number of semiconductor materials. Examples of semiconductors that could be interesting, especially in the visible and NIR region, include indium-tin-oxide, aluminum-zinc- 
oxide and gallium-zinc-oxide ${ }^{14}$.

\subsubsection{MAXWELL'S EQUATIONS}

The interaction of light and matter is governed by a set of classical equations, even at the subwavelength scale. These equations are called Maxwell's equations. They are differential equations that describe the correlation between E-field and $\mathrm{H}$-field of an electromagnetic wave at any point in space-time. Maxwell's equations are critical in understanding electromagnetic properties in the medium. The flow of current produces a magnetic field and a varying current produces a varying magnetic field which in turn gives rise to an electric field. These varying electric and magnetic fields generate a propagating EM wave. The propagation of the wave through a medium is determined by parameters of the medium like the electric permittivity $(\varepsilon)$, magnetic permeability $(\mu)$ and the electric conductivity $(\sigma)$.

Electromagnetic fields are comprised of two vectors, $\mathrm{E}$ and $\mathrm{H}$, for the electric and magnetic components respectively. The existence of propagating electromagnetic fields in an infinite, nonconducting medium with constant permittivity, $\varepsilon$ and permeability, $\mu$ in the direction of the complex wave vector, $\mathrm{k}$ is given by Maxwell's plane wave equations which are

$$
\nabla \cdot \mathrm{E}=\frac{\rho_{v}}{\varepsilon}
$$

Equation (2) is Gauss's law for a static electric field, which states electric flux through any closed surface is proportional to total electric charge enclosed by this surface.

$$
\nabla \cdot \mathrm{H}=0
$$

Equation (3) is Gauss's law for static magnetic fields, which states magnetic flux passing through any closed surface is zero.

$$
\nabla \times \boldsymbol{E}=-\mu \frac{\partial \mathrm{H}}{\partial \mathrm{t}}
$$

Equation (4) is Faraday's law of induction, which states changing magnetic flux through a surface induces circulating Electric field.

$$
\nabla \times \boldsymbol{H}=\mathrm{J}+\varepsilon \frac{\partial \mathrm{H}}{\partial \mathrm{t}}
$$


Equation (5) is Ampere's law, which states a changing electric flux through a surface produces a circulating magnetic field around any path that bounds that surface.

\subsection{IMPORTANCE OF NANOSTRUCTURE ARRAY FOR PLASMONIC APPLICATIONS}

Nanostructured materials have tunable physicochemical characteristics, unlike bulk materials where their properties are limited to its material property. Nanostructures allow tuning the material properties like light absorption, color change, electrical and thermal properties with different sizes and shapes of the structure. Understanding the fundamental optical properties has allowed for plasmonic nanostructure research and development throughout the past two decades. This is due to the growth of advanced fabrication and characterization techniques along with potential applications in the fields of medicine, biosensing, imaging, solar energy, catalysis and optoelectronics.

A plasmon is typically excited on two types of substrates, 1) free-standing individual nanoparticles ${ }^{15}$ and their random aggregates ${ }^{16}$ and 2) chip-based nano-array patterns. Local EM

enhancement factors can reach $10^{4}-10^{6}$ with metal nanospheres ${ }^{17}$. With colloidal aggregates, the EM enhancement factors can achieve as high as $10^{14}{ }^{18}$. Individual nanoparticles have been extensively used for surface-enhanced Raman scattering (SERS). It is noted that the SERS enhancement is difficult to reproduce because of the random distribution of colloids.

A nano-array pattern is a periodic layout of particular nanostructure geometry over a solidstate chip. Many nano-array patterns have been reported to generate tunable a plasmon such as a nanohole, nanocubes, nanorods, nanopyramids and nanorings. In particular, the collective behavior of plasmons in nano-arrays produces a coherent optical response, which leads to stronger and narrow spectral features. The optical spectrum of a nano-array depends on size, shape, and periodicity of the structures, which provides great flexibility and tunability of plasmon and optical properties. The tailorable plasmonic properties in nano-arrays have opened ample opportunities for EM enhancement and light management with nano-arrays, making nano-arrays applicable in photovoltaic devices, photocatalysts, biosensors, nano-medicine, and optoelectronic devices. 
For example, plasmonic nano-arrays have been extensively used for two types of sensors, that is, surface plasmon sensors and SERS sensors. Plasmonic sensors are designed based on the principle that plasmon resonance is sensitive to refractive index changes in the environmental medium due to the adsorption or binding of molecules on the plasmonic nanostructures. Raman scattering is the result of inelastic scattering when light interacts with a molecule, which is used to detect vibrational and rotational properties of a system. The induced electric dipole moment for a Raman molecule is given by ${ }^{19}$

$$
\mu=\alpha E
$$

Where $\alpha$ is the molecular polarizability and $E$ is the electric field strength. The induced electric dipole moment of a molecule is directly proportional to the electric field strength of the medium where the molecule is present. Raman scattering intensity is directly proportional to the square of the induced dipole moment. Therefore, when a Raman molecule is placed in a medium with a high electric field, the Raman signal is enhanced through a phenomenon called SERS. When nanostructures are irradiated with light, the light can be confined in subwavelength dimensions and produce a large EM field for SERS enhancement. Because of the high enhancement factors, SERS is a very promising technique for sensing applications to overcome poor sensitivity and high background interference. Since SERS was first realized for single molecule detection in 1997 [20, 21], its application in the biosensing and nanomedicine fields has grown considerably. Over the past 20 years, the vast majority of SERS sensors have been engineered using colloidal nanoparticle SERS systems. Colloidal nanoparticles in SERS sensors systems are limited for use as in-solution detection probes, which can be subject to unwanted aggregation in biological matrices. Additionally, colloidal nanostructures, such as silver and gold nanorods, have challenging synthesis methods that are difficult to reproduce. Compared to nanoparticles, nano-arrays are highly stable and ordered structures, and can extend the space for light management and EM enhancement. To further improve sensor performance and diversify material design, 2D and 3D nano-arrays have gained interest for SERS sensing substrates.

\subsection{FABRICATION TECHNIQUES FOR NANOSTRUCTURE ARRAY}

Cleanroom-based photolithography (PL) is one of the most widely used and mass fabrication techniques in the semiconductor and microfabrication industry. However, its spatial resolution is limited by the diffraction limit of light. The angle of diffraction depends on the wavelength of light 
used and the gap between a photomask and the substrate. For these reasons, conventional photolithography is unable to produce high-quality nanoscale features, especially for 3D and hierarchical nanostructures. New nano-fabrication techniques have emerged to overcome the challenges associated with photolithography. These lithography techniques include electron-beam lithography, focused-ion lithography, dip-pen lithography, laser interference lithography, nanosphere lithography, nanoimprint lithography, anodic aluminum oxide (AAO) template-based lithography, and molecular stamping. An ideal nanofabrication technique is inexpensive, high throughput, high resolution, and provides great flexibility for tailoring nanostructure size and shape.

\section{Electron-beam lithography (EBL)}

EBL was first developed in $1967^{20}$ and has took many modifications and developments to achieve high spatial resolution. Electron-beam lithography (EBL) is a modified scanning electron microscope (SEM) to write a nanoscale pattern by focusing an electron beam spot on an electron sensitive resist. Projection printing and direct writing are two methods followed in EBL systems ${ }^{21}$. The difference between them is a projection scheme uses a larger electron beam projected on to the mask, where as in direct writing e-beam spot pattern the shape directly on the resist. Generally, there are two sub categories of direct writing EBL systems based on the type of electron beam used. One is Gaussian beam e-beam system and the other is shaped beam e-beam system ${ }^{22}$. Gaussian e-beam systems are commonly used because of its flexibility and fine spatial resolution ${ }^{23}$. This section discusses about the direct writing EBL system. In direct writing EBL, an electron beam spot is focused on electron beam resist coated on a wafer. The pattern is transferred in the form of raster scans. The exposed part of resist is etched away if its positive resist using a developer leaving a nanostructure pattern. The resolution of nanostructure is depended on the size of beam spot, which is around $10 \mathrm{~nm}$. Since the EBL is serial process which uses raster scans the throughput is very low.

\section{Focused ion beam (FIB) lithography}

Ions are used in FIB similar to electrons used in EBL. In FIB heavier ions are projected on to the resist where as in EBL electrons are used. The advantages of using heavier ions improves the resolution $^{24}$. The Debroglie wavelength of heavier ions is lower than electrons which give better 
control of feature dimensions on the resist. However heavier ions like $\mathrm{He}^{+}, \mathrm{Be}^{+}$and $\mathrm{Ga}^{+}$have less penetration depth so high aspect ratio features are difficult to fabrication with FIB. Also, FIB need large energy to focus the ion beam because of the heavier ions. Similar to EBL, FIB also lacks high throughput.

\section{Dip-pen lithography (DPN)}

Dip-pen lithography (DPN) is a type of scanning probe lithography, where an atomic force microscope (AFM) tip is used to directly write a pattern by delivering chemical reagents directly on to the substrate. DPN was first introduced in 1999 as a tool for fabricating nanostructures ${ }^{32}$. Since then, it was developed to pattern variety of inks including organic molecules, colloidal particles, metal ions, and biological polymers ${ }^{33}$. Also, a variety of substrate surfaces can be patterned such as metals, insulators and semiconductors. DNP lithography is conducted in inert environment making this technique an ideal candidate for pattering biological and soft organic structures. DPN offers high resolution as shown in the patterning of alkylthiols on gold substrate to achieve $15 \mathrm{~nm}$ resolution with sharp tips ${ }^{34}$. Recently, parallel writing DPN has been developed, which addresses the complexity of engineering cantilever arrays by using a passive pen array ${ }^{35}$. The main disadvantage of DPN is its throughput because of its inherent serial fabrication process. Generally, DPN uses a single pen to write the pattern, even though efforts have been made to use multi pen configuration. Engineering cantilever array design for specific applications is difficult and expensive. One of the disadvantages of DPN is that limited number of materials can be used in the ink for DPN.

\section{Laser interference lithography (LIL)}

Laser inference lithography (LIL) is similar to mask-less photolithography for fabricating nanostructures. It is an effective method for large area and high throughput fabrication ${ }^{36,37}$. In conventional photolithography, monochromatic light is illuminated through a mask, and transfers a pattern on to the photoresist. In LIL, instead of using a mask to form a pattern, the pattern is formed from the superposition of multiple laser beams exposed onto a photoresist ${ }^{38}$. The interference pattern and electric field intensity is formed during the superposition of multiple laser beams. Because LIL does not use photomask, the patterns of different sizes and shapes can be easily modified by interference principle. Also, the resolution of the feature size is not limited by 
diffraction of light that is commonly seen with mask techniques. The resolution is only limited by wavelength of light being used during the pattern transfer ${ }^{39}$. However, not all shapes can be patterned using LIL and the minimum period of nanostructures is limited to the half the wavelength of light. This requires the usage of deep ultraviolet light for smaller features ${ }^{40}$, which makes the LIL very expensive. For large exposure area, the laser source should have a long coherence length and for higher resolution ${ }^{41}$, lower wavelengths of light are needed. The light source specifications required for desired patterns makes the LIL challenging for large-scale fabrication.

\section{Nano sphere lithography (NSL)}

Nanosphere lithography is a flexible, inexpensive, high throughput technique for fabricating 2D and 3D nanostructures. In NSL, spherical colloids are transferred on to a substrate and dried to form a hexagonally closed pack (HCP) monolayer ${ }^{42}$. The monolayer is transferred on to substrate by various methods like dip coating ${ }^{43,44}$, spin coating ${ }^{45,46}$ and Langmuir-Blodgett ${ }^{47}$. This monolayer is used as a mask and subsequent steps are performed to fabricate different nanostructure array patterns ${ }^{13,48}$. Material is deposited through the interstitial spaces of the HCP beads and then the mask is removed by sonication leaving ordered array of nanostructures on a substrate. The size and shape of the nanostructure can be easily changed by changing the bead size. NSL is a hybrid of top-down and bottom-up approach, which offers a flexible fabrication. Lithographic techniques like EBL, LIL, IBL are low throughput and have high sample costs making these techniques impractical for large-scale fabrication. Because of these challenges, many parallel lithographic techniques have been developed, with NSL among them. NSL is a promising technique for developing nanoscale substrates because of the low cost, high throughput, and tunable optical properties ${ }^{49-51}$. However, since a HCP monolayer is used as the mask for lithography, the shape and size of nanostructure features are limited, so it is challenging to generate versatile nanostructure geometries and features.

\section{Nanoimprint lithography (NIL)}

Nano imprint lithography (NIL) is a high resolution, high throughput, and low cost technique for fabricating nanostructures. It was first developed by S.Y. Chou in $1995^{52}$, and since then it has overcome many challenges to meet the practical industrial requirements. NIL has demonstrated sub $10 \mathrm{~nm}$ imprinting in $1997^{53}$ and $2 \mathrm{~nm}$ structures fabrication ${ }^{54}$, making it a promising technique 
for next generation lithography. It has been added to the International Technology Roadmap for Semiconductors (ITRS) for $32 \mathrm{~nm}$ and $22 \mathrm{~nm}$ nodes ${ }^{55}$.

NIL uses molds to form nanostructures. In this technique, a stamp (or mold) with a specific pattern is mechanically pressed into the imprint fluid already coated on a substrate. The mechanical deformation causes the pattern to be transferred into the imprint fluid. After the hardening process, the stamp is removed and leaves the nanostructure pattern on the substrate. The resolution of NIL is limited by the mold pattern, and is not due to the diffraction limit of light such as in the case of photolithography. Therefore, the minimum feature size of the nanostructure pattern depends on the mold pattern feature size. Based on the type of imprint curing, there are two commonly followed nanoimprint processes thermal NIL and UV-NIL ${ }^{56}$. Thermal NIL, the earliest form of NIL, utilizes a thermoplastic polymer as the imprint fluid. During the hardening step, the polymer is heated above the glass transition temperature to transfer the pattern. In case of UV-NIL, a UV sensitive polymer is used as an imprint fluid. After the mechanical contact, required dosage of UV light is illuminated on to the polymer to complete the hardening process. The applications of NIL are categorized into pattern transfer and polymer device applications. Its main applications are in optical storage devices, hard disk media devices, light emitting diodes, biosensors, microfluidic devices and functional polymer devices.

\section{Fabrication with anodic aluminum oxide (AAO) and other templates}

Anodic aluminum oxide (AAO) template is a template assisted nano-patterning technique for low cost and high resolution ${ }^{57}$ over a large area. The subsequent deposition of material through the template allows for fabrication of nanostructures. The template is made by metal anodization in an acidic solution to form periodic metal oxide nanopores ${ }^{58}$. The thickness of the membrane and the periodicity of pores depend on time of anodization and the voltage applied ${ }^{59}$. The flexibility of fabricating pore size which ranges from $7 \mathrm{~nm}$ to more than $300 \mathrm{~nm}$ make AAO a suitable template assisted pattering for high resolution fabrication ${ }^{60}$. This technique is applied to fabricate several nanostructures such as nano dots, rings, pillars and tubes over a large area. Besides aluminum, titanium and zirconium metals can also be used to generate pore patterns by anodic etching. However, it is difficult to generate long-range-ordered periodic nano-array patterns in a large area. Furthermore, the geometry and layout of the nanostructure feature are limited by the fixed AAO template because the template pores are round-shaped and laid out in a hexagonal pattern. 


\subsection{PLASMON MODES in 2D AND 3D NANOSTRUCTURES}

Similar to photons in a light wave, plasmons are quasiparticles of an oscillating plasma composed conduction electrons at the boundaries between metal and dielectric ${ }^{68-71}$. The electric field vector of light oscillates free electrons at frequency to create a plasma. When the excitation light frequency matches the electron oscillations, plasmon resonance occurs. Materials with negative real and positive imaginary dielectric constant support surface plasmon resonance (SPR) when they are illuminated. The incident light couples with the plasmons, which either strongly scatters or absorb light. Understanding the relationship between material properties like size, shape, periodicity and dielectric with the optical response is being explored and has resulted in wide range of applications including SERS ${ }^{72,73}$, sensors ${ }^{74,75}$, fluorescence enhancement ${ }^{76}$, and refractive index measurements ${ }^{77}$. Surface plasmon oscillations vary based on the nanostructure properties and dielectric environment. These different types of surface plasmon oscillations are called plasmon modes. Plasmon modes can be modulated based on the suited applications and the desired physical properties. Plasmon modes differ in fundamental properties such as near-field electric field, mode volume, and bandwidth. SPR occurs in two fundamental forms localized surface plasmon resonance (LSRP) and surface plasmon polariton (SPP). Other plasmon modes, such as Fano resonance, plasmonic whispering-gallery mode, surface lattice resonance and plasmonic gap mode, can be excited from the coupling and interaction of these fundamental modes by controlling the nanoarchitecture.

Each plasmonic mode has characteristic optical properties and unique distribution and amplitude of EM field. It is essential to understand the origin of plasmon excitation and decay, the optical responses of plasmon, and the associated effects such as hot electron emission in order to fabricate and utilize plasmonic nano-arrays in a fashion of "device-by-design". For example, for the application of plasmonic nano-array in plasmon sensors and SERS sensors, a well-designed nanoarray pattern along with the fundamental understanding of light interaction with nano-array pattern is required. 


\section{Localized surface plasmon resonance (LSPR):}

Localized surface plasmon resonance (LSPR) is a surface phenomenon generated by metal nanoparticles, in which electrons oscillate collectively in resonance with incident light (Figure 1A). They are localized at particular areas on the nanostructure as localized plasmons. The electric field around the localized plasmons is multiplied by several orders of magnitude, and intensity decays exponentially with distance. These resonances create a sharp optical absorption or scattering along with strong EM field.
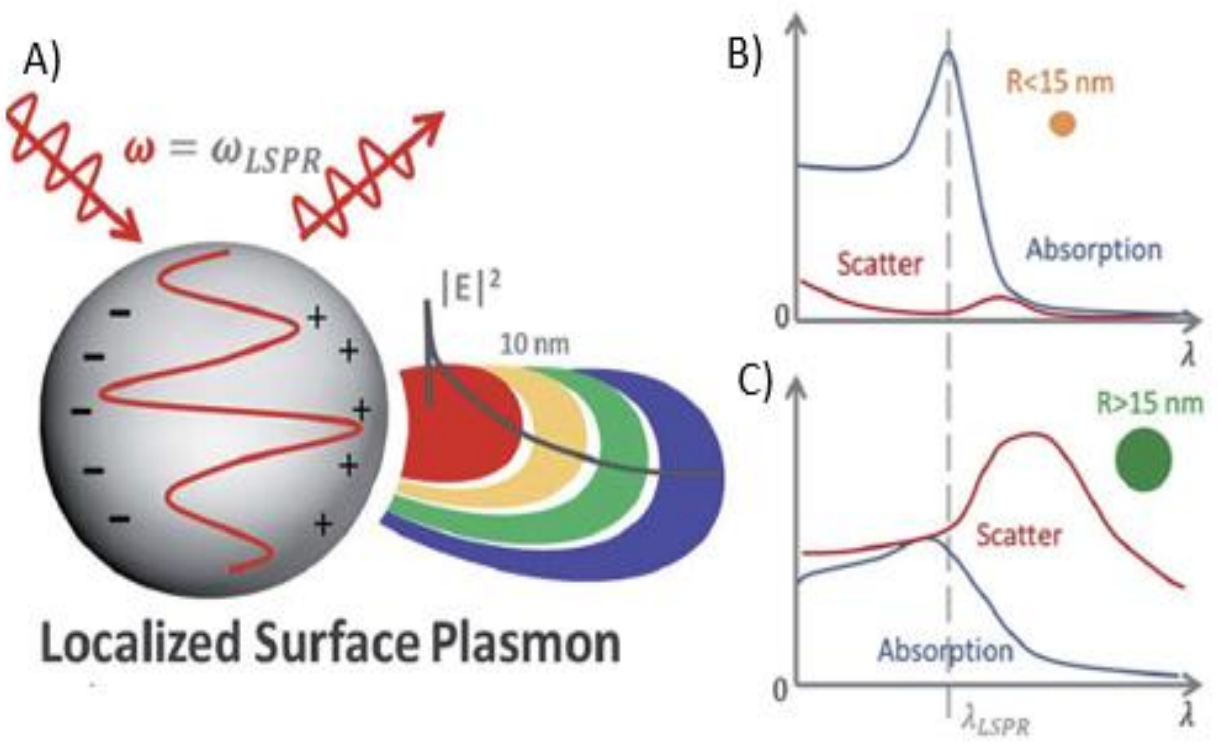

Figure 2. 1: (A) LSPR condition and E filed distribution (B) Absorption dominates in smaller nano particles $(C)$ Scattering dominates in larger nano particles ${ }^{78}$

The LSPR peak is highly dependent on the material, dielectric constant of surrounding medium, and size of nanostructure as given by the above equation. The real part of dielectric function determines the LSPR extinction peak position, while the imaginary part plays a role in the damping and resonance peak broadening. When $\boldsymbol{\varepsilon}_{l}=-2 \boldsymbol{\varepsilon}_{m}$, polarization attain singularity and EM field is enhanced. Mie's theory is limited only to spherical particles. In 1912, Richard Gans generalized Mie's equation based on small particle approximation and found the absorption crosssection for prolate spheroid as [80] 


$$
\sigma_{a b s}=\frac{\omega}{3 c} \varepsilon_{m}^{3 / 2} V \frac{\left(\frac{1}{P_{j}^{2}}\right) \varepsilon_{2}}{\left\{\varepsilon_{1}+\left[\frac{1-P_{j}}{P_{j}}\right] \varepsilon_{m}\right\}^{2}+\varepsilon_{2}^{2}}
$$

This gives extinction spectrum from both transverse plasmon mode and longitudinal plasmon mode. This equation shows the dependency of shape on LSPR peak wavelength, by incorporating shape dependent dielectric function $\frac{\left(1-P_{j}\right)}{P_{j}}$. For the shapes other than spheres and spheroids, LSPR spectrum is studied numerically with Finite Difference Time Domain (FDTD) calculations. For noble metals such as Ag and Au nanoparticles, singularity condition $\left(\boldsymbol{\varepsilon}_{1}=-2 \boldsymbol{\varepsilon}_{m}\right)$ is met in visible region and near-infrared where optoelectronic devices are operated and many biological applications are conducted. This gives noble metals an advantage over other materials for sensing devices. The sharp optical response and local EM field of LSPR phenomenon are used in many applications like biomolecule sensing, imaging, metamaterials, and resonance energy transfer.

The local EM filed of LSPR is directly proportional to the SERS enhancement. Thus, to improve the sensing performance, a SERS substrate is optimized with large density of localized plasmons known as plasmonic "hot spots" ${ }^{81,82}$. Hot spots are areas in the vicinity of the plasmonic nanostructures where the local EM filed is enhanced enormously in comparison to its surroundings. Typically, hot spots are formed at the sharp tips of nanostructures where there is large charge density. As the distance between the metal nanostructures decreases, the EM enhancement at the hotspot increases exponentially ${ }^{82}$. When a Raman molecule is located in the hot spot, there is a tremendous enhancement in Raman signal. Typically, SERS enhancement is in the order of $10^{4}-10^{8}$, but nano-array substrates with high density hot spots have enhancement ranging from $10^{8}-10^{12} 84,85$.

\section{Surface plasmon polariton (SPP)}

Surface plasmon polaritons (SPPs) are surface EM waves that propagates along the metaldielectric interface. These waves are generally visible or infrared waves whose EM field decay with distance dissipating energy into both medium ${ }^{104}$. Unlike LSPR, SPP cannot be excited directly by incident light due to high momentum requirement. The classic example of SPP is the Kretschmann configuration where a gold film is coupled to a prism and demonstrates optical 
excitation of SPP on metal surface ${ }^{105,106}$. The prism with large refractive index enables an evanescent field through attenuated total reflection that excites plasmons. The prism makes the horizontal wave vector components of the incident light couple to the propagating SPP wave vector, as long as the two wave vectors match at a certain incident angle (Figure 3D).

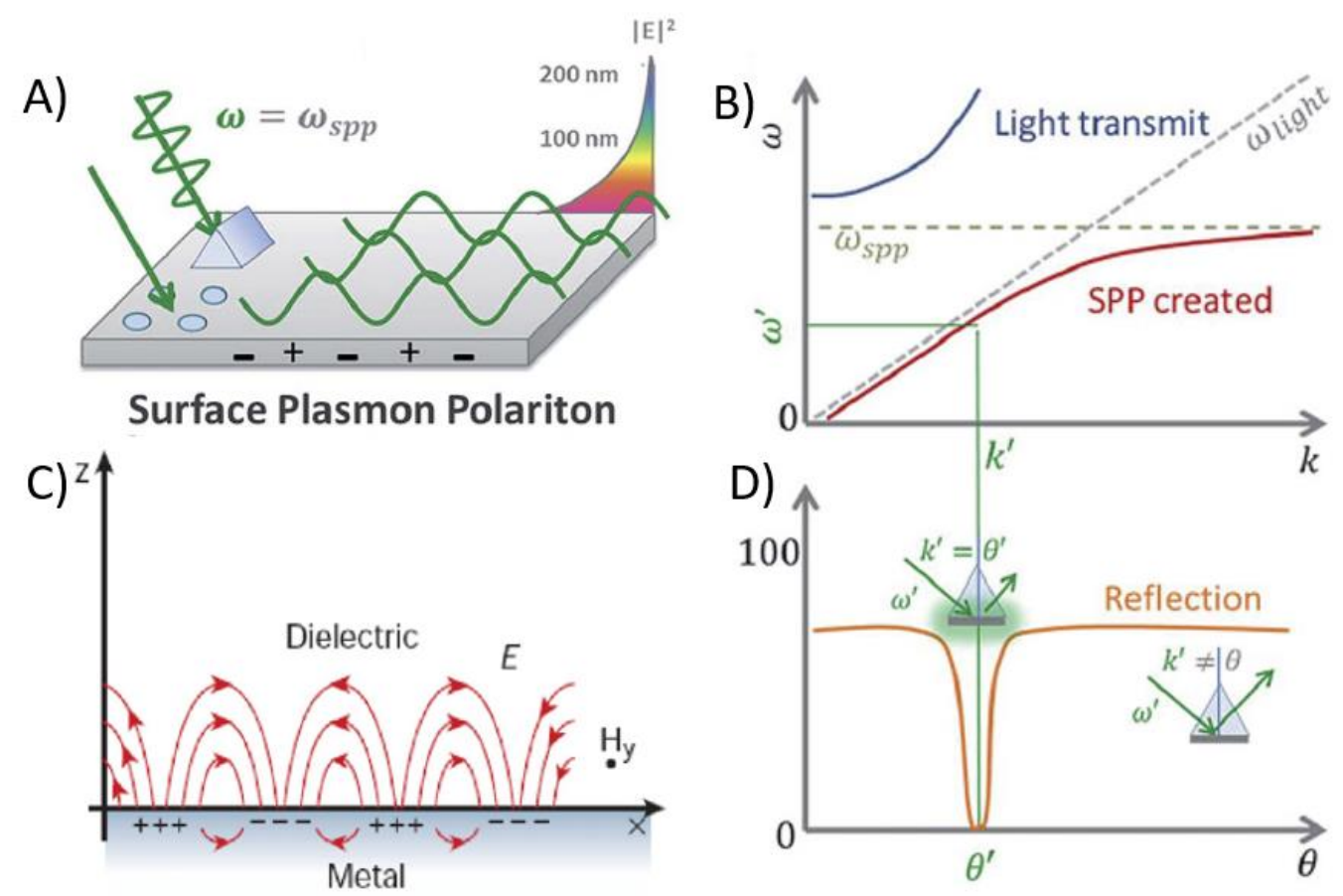

Figure 2. 2: (A) SPP propagating wave (B) Wave momentum match condition $(C)$ Penetration depth of SPP into metal and dielectric medium (D) Resonance frequency match with angle , 78, 107

From Maxwell equations, the SPP dispersion relation can be expressed as $[108,109]$

$$
\mathrm{K}_{\mathrm{spp}}=\mathrm{k} \sqrt{\frac{\varepsilon_{d} \varepsilon_{m}}{\varepsilon_{d}+\varepsilon_{m}}}
$$

The nonlinear characteristic nature of SPP results in a momentum mismatch between light and SPP. This mismatch can be overcome by coupling light and SPP modes at the condition

$$
\varepsilon_{d}+\varepsilon_{m}=0
$$


At this condition, the real part of the dielectric constant is negative and denominator in the above

equation is zero resulting to a resonance condition with resonance frequency $\frac{\omega_{p}}{\sqrt{1+\varepsilon_{d}}}$, where $\omega_{p}$ is the bulk plasma frequency. This resonance generates a surface plasmon propagating wave along the metal-dielectric interface and decays exponentially with distance. The penetration depth of EM field in dielectric is longer than the depth in metal and this difference is more prominent at longer wavelengths ${ }^{110}$.

The distinct behavior of SPPs, which allows control of light in nanometer scale, opened a potentially interesting applications in photonics ${ }^{111,112}$, SERS $^{113-115}$, spectroscopy $^{116}$, data storage ${ }^{117}$ and in sensors ${ }^{118-119}$. Extraordinary optical transmission (EOT) is a phenomenon in which at a particular wavelength range transmission of light is greatly enhanced in a subwavelength aperture due to the excitation of SPP in an opaque metallic film in the presence of nanohole array ${ }^{111}$

\section{Fano resonance}

Fano resonance is the interaction between a narrow discreet state and a broad continuum, producing an asymmetric spectral shape with resonant suppression and enhancement in a narrow window of spectrum ${ }^{131}$. The resonance enhancement is the consequence of two modes that interfere constructively, while resonance suppression is due to destructive interference of such modes. The fundamental spectral result of a resonance from one or more independent resonances is given by Lorentzian formula, which describes superposition of individual resonances. Because Ugo Fano has discovered a new type of resonance unlike Lorentzian resonance with an asymmetric shape in his study of autoionizing states of atoms, this type of resonance is called Fano resonance. The absorption spectrum shape of Fano resonance is given by ${ }^{132}$

$$
\sigma(E) \alpha \frac{(q+\xi)^{2}}{\left(1+\xi^{2}\right)}
$$

Where $E$ is the energy, $q$ is the shape parameter that determines the asymmetry of the profile, and $\xi$ is $\frac{2(E-E 0)}{f}$

where $E_{0}$ and $\mathrm{f}$ are energy and resonance width respectively.

A Fano resonance can be derived from Mie theory for a single spherical plasmonic particle. The magnetic and electric amplitudes depend on size parameter. Considering small size $\mathrm{q}<<1$ and non-magnetic particle $(\mu=1)$, Rayleigh scattering yields ${ }^{133}$. 


$$
Q_{s c a}=\frac{8}{3} \frac{|\varepsilon-1|^{2}}{|\varepsilon+2|} q^{4}
$$

With increase in size $(q>1)$, number of eigen modes increases creating extra resonances. When $\varepsilon>1$, all the resonances are broad and there is no possibility of Fano resonance. When $\varepsilon<0$ the resonance is broad at the same time for $\varepsilon<0$ and $q>1$ the resonance is weakly damped for dissipative materials (noble metals) and have a narrow resonance. These two resonances coexist over a window of ' $\varepsilon$ ' forming Fano resonance.

\section{Discrete state}

A)

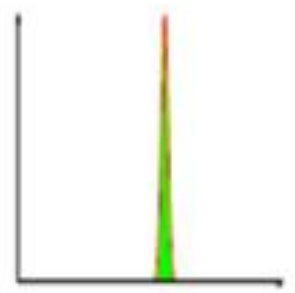

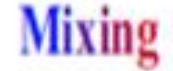

B)
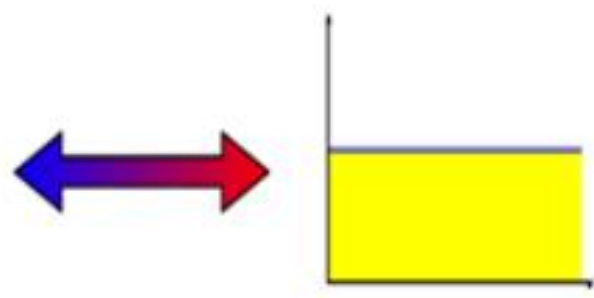

Fano resonance

C)

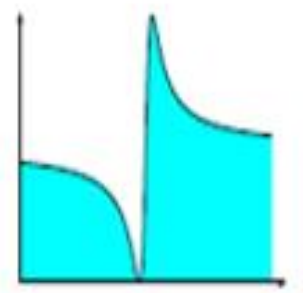

Figure 2. 3: Illustration of Fano resonance ${ }^{134}$

The Fano resonance phenomenon exists in plasmonic nanostructures ${ }^{136-138}$, where the plasmon resonance usually a continuum and couples with a discrete state to form a Fano resonant mode (Figure 5C).

\section{Plasmonic whispering-gallery modes (WGM)}

Whispering gallery mode resonances occur when an electromagnetic field becomes trapped at the surface of a nanostructure due to total internal reflection. They are gallery modes specific to a cavity and highly dependent on the geometry of the cavity ${ }^{153}$. WGM resonators have a high quality factor $(\mathrm{Q})$, making them a potential candidate for biosensors. When the length of optical path matches the integer number of wavelength, a standing wave is formed inside the cavity. At this resonance condition, the mode is confined in the dielectric medium, and a small portion extends outside, which is very sensitive to the surrounding environment. The change in the resonant frequency with molecule is very sensitive and used as WGM sensors. The performance of a WGM resonator is measured by a quality factor $(\mathrm{Q})$, defined as 


$$
\mathrm{Q}=\frac{\omega_{0}}{\Delta \omega_{F W H M}}
$$

where $\omega_{0}$ is the angular resonance frequency and $\Delta \omega_{F W H M}$ is linewidth of the frequency.

\section{Surface lattice resonance (SLR)}

Surface lattice resonance arises from optical coupling of LSPR modes in arrays of metallic nanoparticles (Figure 9) ${ }^{169-170}$. Compared to LSPR, SLR has much narrower optical spectrum features, making this mode desirable for light absorption applications and sensing ${ }^{171}$. Like LSPR, the SLR modes can be tuned along the UV-Vis and NIR spectrum by variation in morphology, particle size, material, refractive environment and periodicity ${ }^{169-171}$.

A)
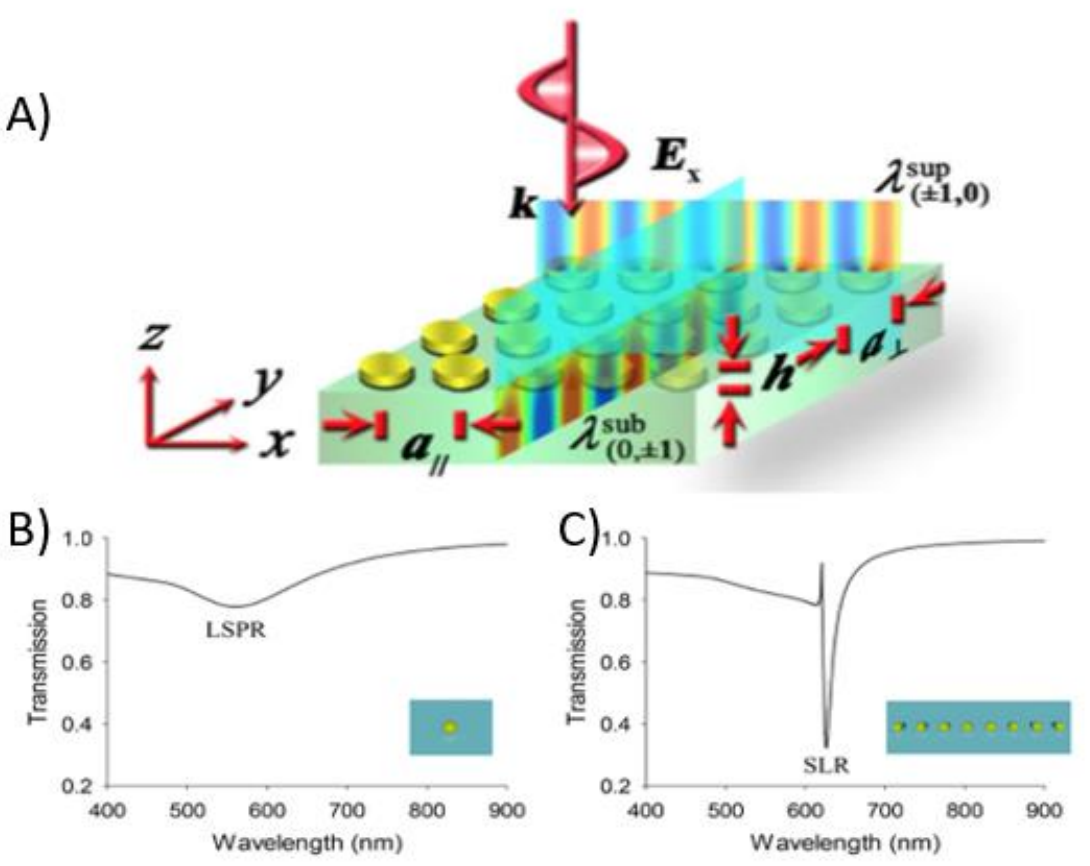

Figure 2. 4 (A) Surfac Lattice resonance illustration ( $B \& C$ ) Comparision of LSPR and SLR transmission spectra ${ }^{169,184}$

The coupled dipole approximation (CDA) is instrumental in understanding the difference in extinction cross section between LSPR and SLR. In this approximation for SLR, the dipole sum provides an additional degree of freedom (dependent on periodicity, particle size, etc.) to govern 
the width of SLRs mode ${ }^{169}$. The effective polarizability $\left(\alpha_{e f f}\right)$ and the extinction cross section $\left(C_{\text {ext }}\right)$ are given by

$$
\begin{aligned}
& \alpha_{\text {eff }}=\frac{1}{\frac{1}{\alpha_{s}}-S} \\
& C_{\text {ext }}=4 \pi N k \operatorname{Im}\left(\alpha_{\text {eff }}\right)
\end{aligned}
$$

Where $\alpha_{s}$ is the isolated nanoparticles polarizability, $S$ is the retarted dipole summation from other particles, $N$ is the number of particles, and $k$ is the wavenumber of incident light.

Nano-arrays are designed with assistance from CDA to support SLR. The primary control parameters to realize SLR are particle size and lattice constant as they control the coherent interaction of the plasmons ${ }^{169-172}$. An increasing lattice constant results in a more negative dipole sum, which redshifts the resonant frequency and decreases Full width at half maximum (FWHM) while decreasing diameter will blue shift in resonant frequency. SLR supports both orthogonal and parallel EM coupling, which is dependent on relationship between incident light propagation and diffraction wave propagation. Orthogonal or parallel coupling occurs when the light polarization is either vertical or parallel to the diffraction wave. These characteristics allow for SLR modes to be easily tuned to desired wavelengths along the EM spectrum.

\section{Gap plasmon}

Surface plasmons that exist between two metal structures and confine high electromagnetic energy in such small gap is generally called as gap plasmons (Figure 11). When the metal structures are in close proximity, the near-field coupling dominates confining the EM field in the nanogap. When the gap increases, the coupling weakens and properties of individual metal structure are displayed. Recently, planar arrays of nanostructures coupled with film separated by a spacer layer ${ }^{185-187}$ have increasing attention because of its controlled EM over large tunable wavelength range. The high enhancement of plasmon intensity at the gap is a subject of interest because of its applications in metamaterials, energy transfer, sensors and solar energy harvesting. 


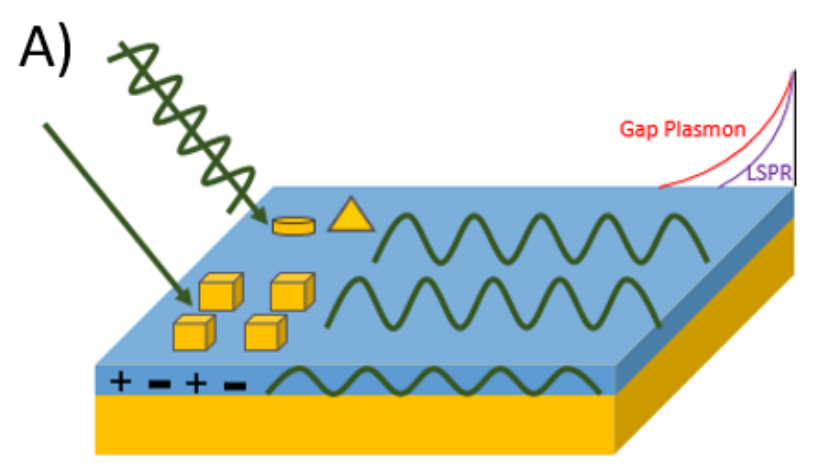

B)

Gap Plasmon

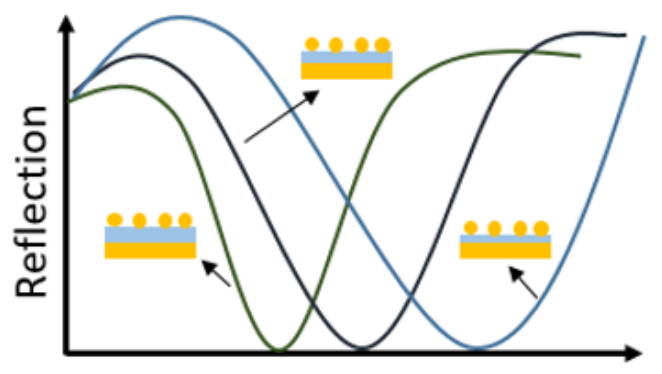

Figure 2. 5: (A) Gap plasmon standing wave illustration (B) Gap plasmon resonance tuning with spacer layer thickness

There are two prominent multilayer configurations where gap plasmon can be realized. One is MIM and the other is insulator-metal-insulator (IMI). Both structures confine the gap plasmon, but MIM configuration is superior in terms of mode volume, strong confinement and quality factor $^{188-189}$. In a simple MIM structure, the metal on the illumination side should be thin enough to allow the incident light to enter through the system. Once the light pass through the metal strip, light gets trapped in the dielectric layer, forming a standing wave called as gap plasmon shown in (Figure 11A). This shows a strong absorption peak in the spectrum. This can be explained by Fabry-Perot (FP) formula ${ }^{193}$

$$
w \frac{2 \pi}{\lambda} n_{e f f}=p \pi+\phi
$$

Where $w$ is the strip width, $\mathrm{p}$ is an integer, $\lambda$ is the wavelength, $n_{\text {eff }}$ is the effective refractive index where gap mode is formed, $\phi$ is an additional phase shift due to reflection. By tuning the spacer layer thickness, and refractive index absorption peak is tuned over a large wavelength range (Figure 11B). Gap mode is very sensitive to the spacer layer thickness. When the space layer is very thin (typically $<8 \mathrm{~nm}$ ), the gap plasmon is very intense due to the strong light confinement. The EM field strength of the gap plasmon decreases with an increase in the thickness of the space layer. So there is a range of thickness a MIM can support a gap plasmon mode. 


\subsection{SURFACE ENHANCED SPECTROSCOPY}

Surface-enhanced Raman spectroscopy (SERS) was discovered in 1974 by Fleischmann, Hendra, and McQuillan ${ }^{25}$. They discovered that the Raman spectrum of pyridine on a silver electrode showed strong Raman intensity. The strength of the induced dipole moment for a Raman active molecule is given by $\mu=\alpha \mathrm{E}$, where $\alpha$ is the molecular polarizabity and $\mathrm{E}$ is the electric field strength. So, the dipole moment can be increased with electric field strength, in turn shows high response in Raman spectroscopy which is enhanced by surface of metal nano particles. Electromagnetic enhancement is the dominant effect and occurs due to the enhancement of the electromagnetic field near the molecule of interest. This electromagnetic enhancement is caused by the interaction between the electromagnetic field and a nearby metallic substrate. The second form of enhancement is referred to as chemical enhancement and is a result of a change in polarizability induced by charge transfer and bond formation between the metallic substrate and the molecule of interest ${ }^{26}$

\subsubsection{SURFACE ENHANCED RAMAN SCATTERING}

Raman scattering is a type of secondary radiation that occurs when light interacts with and is scattered by molecules. This light scattering phenomenon was first discovered in 1928 by Chandrasekhara Venkata Raman ${ }^{27}$. Raman's observations were influenced by the early work of Lord Rayleigh, who had reported on elastic light scattering in the atmosphere in $1871^{28}$. It is a technique used to detect vibrational and rotational properties of a system. Each molecule has a fingerprint by which it can be detected using Raman spectroscopy. The inelastic scattering of light from molecule results in a change in energy of incident light. Scattered photons can be either of lower energy (stokes) or higher energy (anti-stokes), which gives information about vibrational modes in the system ${ }^{29}$. Rayleigh (or elastic) scattering is the dominant light scattering effect and results when light is scattered off molecules with no energy shift. Raman (or Inelastic) light scattering is a comparatively weak scattering phenomenon that occurs because of molecular vibrations. In Raman scattering, photo energy is transferred as it interacts with the molecules, causing a shift in the wavelength of the scattered light. 


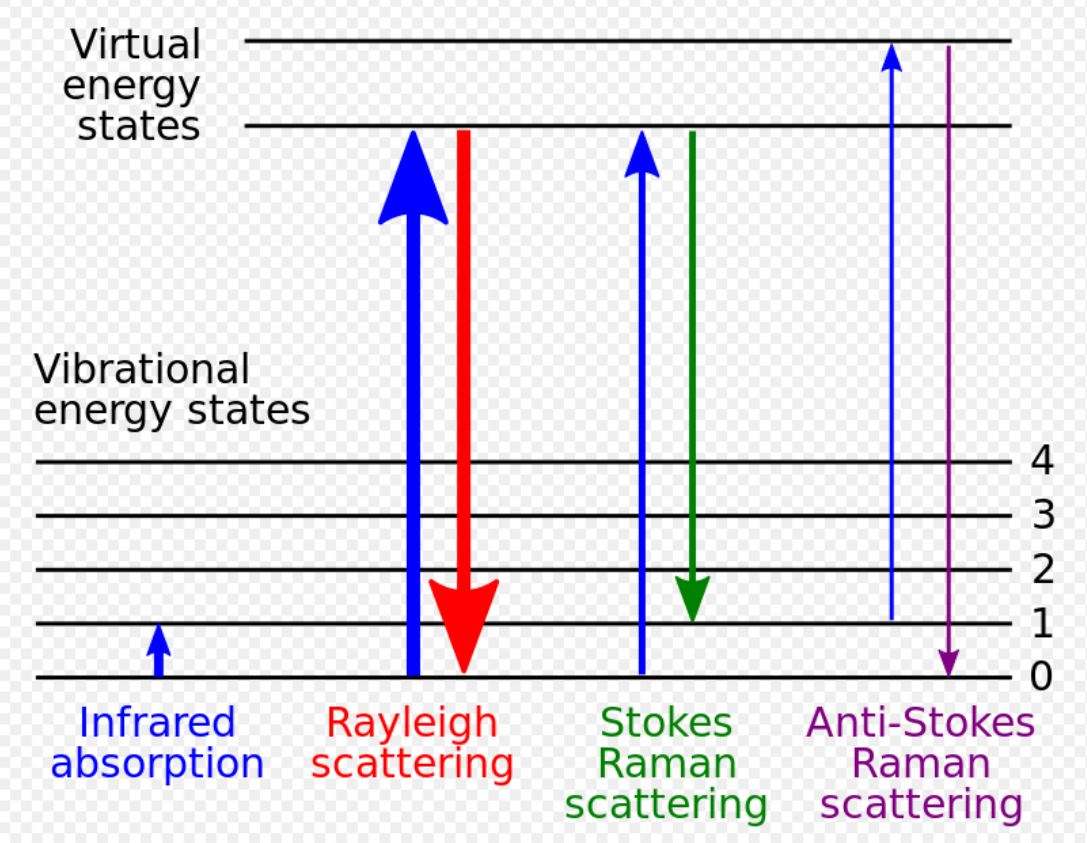

Figure 2. 6: Energy level diagram of Raman spectra ${ }^{29}$

\subsection{PLASMON ENHANCED FLUORESCENCE}

Plasmon Enhanced Fluorescence (PEF) is similar to Surface Enhanced Raman Scattering (SERS) and had tremendous growth in recent years. Due to unique properties, PEF is the most spread optical method. In PEF, the fluorophore is allowed to couple with EM field of surface plasmon to enhance emitted fluorescence light intensity. 


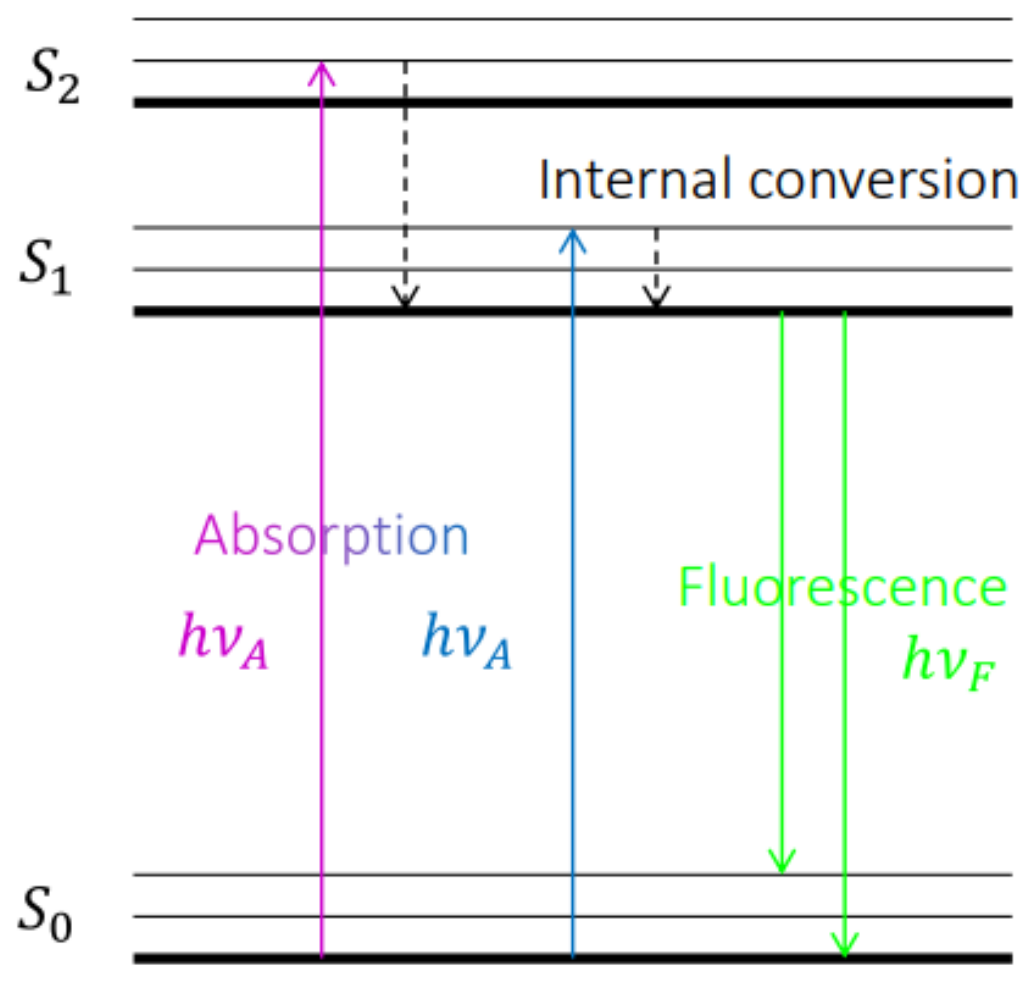

Figure 2. 7: Jablanski diagram for fluorescence ${ }^{30}$

Fluorescence is luminescence occurred when monochromatic or white light is illuminated on a fluorophore. A fluorophore absorbs light at lower wavelengths and emits light at higher wavelengths, these two are called excitation wavelength and emission wavelengths respectively. The high energy light excites the system promoting electrons within the molecule to unstable state and eventually return to stable state emitting light in the form of fluorescence. The optical excitation cross section of nanostructures is many order of magnitude higher than molecular fluorophores. Also, the LSPR of nanostructure enables enhanced optical processes. So, when the coupling between the emission wavelength of fluorophore with plasmon resonance of metal nanostructure, there is an enhanced florescence.

There are advantages for PEF over SERS which makes PEF more sensitive and unique. The cross section of fluorescence is much larger than Raman Scattering. The cross section for fluorescence is 9 to 13 orders of magnitude higher than Raman scattering. So, the fluorescence sensitivity in detection is high compared to Raman. Fluorescence has a two-step process where fluorophore absorbs light at shorted wavelengths and emits light at longer wavelengths which 
makes PEF advantageous in detection. Florescence emission is either an enhancement or quenching effect, this depends on probability for the excited carriers to radiatively decay which in turn depends on number of available local density of optical states, which is nothing but Purcell factor. If the non-radiavtive decay is more then we call it as quenching. So it is always challenging to engineer PEF using nanostructure coupling.

\subsection{LIGHT MANAGEMENT APPLICATIOS USING NANOSTRUCTURES}

Light management is so important in improving the performance of optoelectronic devices like solar cells, photodetectors and LEDs. Using high efficient optoelectronic devices not only improve energy conversion but also produce clean energy. The efficiency of an optoelectronic device largely depends on the shape and size of nanostructure. Here, light can be manipulated in subwavelength structures either to scatter, trap or transmit through the nanostructure used in the application. For example, in LEDs the nanostructure design should scatter light so that photons are extracted from the active region efficiently. Similarly, for solar energy applications light should be trapped in the materials and to take longer path to improve the charge carrier generation. Thus,

engineering nanostructures for specific application requires knowledge of how light interact with matter.

The size, shape and structural configuration of nanostructure dictates the light management in a device. Geometry of a structure plays a crucial role; light absorption is enhanced when the size of structure is less than the wavelength of light whereas when size of structure is larger than the wavelength of light then the light scatters or reflects. 


\section{References}

[1] Melosh. Energy and Electron Transfer. Retrieved from URL (http://web.stanford.edu/group/melosh/plasmonics.html)

[2] Murray W A, Astilean S, and Barnes. W L. Transition from localized surface plasmon resonance to extended surface plasmon-polariton as metallic nanoparticles merge to form a periodic hole array, Phys. Rev. B 2004, 69, 165407, 1-7

[3] Fang Y, Sun M. Nanoplasmonic waveguides: towards applications in integrated nanophotonic circuits. Light. Science \& Applications, 2005, 4, 294

[4] Jeanmaire D L, Van Duyne R P. J. Surface Raman spectroelectrochemistry Part 1: Heterocyclic, aromtatic, and aliphatic amines absorbed on the anodized silver electrode, Electroanal. Chem., 1977, 8, 1-20.

[5] Albrecht M G. Creighton Anomalously intense Raman spectra of pyridine at a silver electrode. JA.J. Am. Chem. Soc.1977, 99, 5215-5127.

[6] Haes A J, Van Duyne R P. A Nanoscale Optical Biosensor: Sensitivity and Selectivity of an Approach Based on the Localized Surface Plasmon Resonance Spectroscopy of Triangular Silver Nanoparticles, J Am Chem Soc 2002, 124, 10596-10604.

[7] Hirsch L R, Jackson J B, Lee A, Halas N J, West J L. A Whole Blood Immunoassay Using Gold Nanoshells Anal Chem 2003, 75, 2377-2381.

[8] Dutta A, Naldoni A, Malara F, Govorov A O, Shalaev V, Boltasseva A. Gap-plasmon enhanced water splitting with ultrathin hematite films: The role of plasmonic-based light trapping and hot electrons. Faraday Discuss. 2019, Advance Article.

[9] Zheng P, Kasani S, Wu N. Converting plasmonic light scattering to confined light absorption and creating plexcitons by coupling a gold nano-pyramid array onto a silica-gold film, Nanoscale Horiz. 2019, Advance Article.

[10] M. A. Ordal, L. L. Long, R. J. Bell, S. E. Bell, R. R. Bell, R. W. Alexander and C. A. Ward, Appl. Opt., 1983, 22, 1099-1119.

[11] S. D. Hudson and G. Chumanov. Bioanalytical applications of SERS (surface-enhanced Raman spectroscopy. Anal Bioanal Chem. 2009 Jun;394(3):679-86

[12] Adrien Lalisse ,Gilles Tessier, Jérome Plain, Guillaume Baffou, Plasmonic efficiencies of nanoparticles made of metal nitrides (TiN, ZrN) compared with gold, Scientific Reports,2016, 6,38647 . 
[13] Rowe, D. J.; Jeong, J. S.; Mkhoyan, K. A.; Kortshagen, U. R. Phosphorus-Doped Silicon Nanocrystals Exhibiting Mid-Infrared Localized Surface Plasmon Resonance. Nano Lett. 2013, $13,1317-1322$.

[14] West, P.R. et al. Searching for better plasmonic materials. Laser Photonics Rev, 2010, 4, 795808

[15] Henglein A. Small particle research: physicochemical properties of extremely small colloidal metal and semiconductor particles, Chem Rev 1989, 89, 1861-1873.

[16] Blatchford C G, Campbell J R, Creighton J A. Plasma resonance-enhanced Raman scattering of absorbates on sold colloids: the effects of aggregation. J. A. Surf. Sci. 1982, 120, 435-455.

[17] Xu H et al. Electromagnetic contributions to single-molecule sensitivity in surface-enhanced Raman scattering, Phys. Rev. E 62, 3, 4318-4324.

[18] Kneipp K, Kneipp H, Manoharan R. et al. Extremely large enhancement factors in surfaceenhanced Raman scattering for molecules on colloidal gold clusters, Appl. Spectrosc 1998, 52, 1493-1497

[19] Raman C V. A new radiation. Indian J. Phys. 1928, 2, 387-398

[20] T. H. P. Chang, Ph.D. thesis, Cambridge University, 1967.

[21] Electron Beam Lithography in Nanoscale Fabrication: Recent Development, IEEE trans. electron. packag. manuf, VOL. 26, NO. 2, APRIL 2003.

[22] E-beam lithography for micro-/nanofabrication biomicrofluidics,2010, 4, 0265032010

[23] M. S. M. Saifullah, T. Ondarcuhu, D. K. Koltsov, C. Joachim, and M. E. Welland, Nanotechnology, 2002, 13, 659.

[24] Arshak K, Mihova M. State-of-the-art focused ion beam nanolithography J Optoelectron Adv M, 2005, 7, 1, $193-198$.

[25] Fleischmann M, Hendra PJ, McQuillan AJ.Raman spectra of pyridine adsorbed at a silver electrodeChem Phys Lett,1974, 26:163-166.

[26] Campion A, Ivanecky JE, Child CM, Foster M.On the mechanism of chemical

[27]. Raman CV, Krishnan KS.A new type of secondary radiation, Curr Sci, 1998, 74:381-381.

[28]. Strutt JW.On the light from the sky, its polarization and colour Philos Mag Ser 41871, 41:107-120. 
[29] Wikipedia contributors. (2019, July 20). Raman spectroscopy. In Wikipedia, The Free $\begin{array}{llllll}\text { Encyclopedia. } & \text { Retrieved 15:03, July 22, 2019, from }\end{array}$ (https://en.wikipedia.org/w/index.php?title=Raman_spectroscopy\&oldid=907152717)

[30] Jabłoński, Aleksander "Efficiency of Anti-Stokes Fluorescence in Dyes" Nature 1933, volume 131, pp. 839-840.

[32] Piner R D, Zhu J, Xu F, Hong S H, Mirkin C A, “Dip-pen” lithography Science 1999, 283, 661-663.

[33] Ginger D S, Zhang H, Mirkin C A.The Evolution of Dip-Pen Nanolithography. Angewandte Chemie - International Edition 2004, 43, 30-45.

[34] Mirkin C A. The Power of the Pen: Development of Massively Parallel Dip-Pen Nanolithography, ACS Nano, 2007, 1, 79-83.

[35] Salaita K, Wang Y, Fragala, J, Vega RA, Liu C, Mirkin, C. A. Massively Parallel Dip-Pen Nanolithography with 55,000-Pen Two-Dimensional Arrays. Angew. Chem., Int. Ed. 2006, 4, $7220-7223$.

[36] Roy S. Fabrication of micro- and nano-structured materials using mask-less processes, J. Phys. D: Appl. Phys. 2007, 40, 413-426.

[37] Rodriquez A, Echeverria M, Ellman M et al. Laser interference lithography for nanoscale structuring of materials: From laboratory to industry. Microelectronic Engineering 2009, 86, 4-6, 937-940

[38] Moon J H. Multiple-exposure holographic lithography with phase shift, Appl. Phys. Lett. 2004, 85, 4184.

[39] I Byun and J Kim J. Cost-effective laser interference lithography using a $405 \mathrm{~nm}$ AlInGaN semiconductor laser. Micromech. Microeng, 2010, 20, 055024.

[40] Helgert M, Burkhardt M, Rudolf K, Steiner R, and Brunner R, High-frequent structures generated by interference lithography in the DUV, Frontiers in Optics 2004/Laser Science XXII/Diffractive Optics and Micro-Optics, 2004, 779-2.

[41] O'Reilly T B, Smith H I. Linewidth uniformity in Lloyd's mirror interference lithography systems J. Vac. Sci. Technol. B 2008, 26, 2131-4

[42] Pieranki P. Two-Dimensional Interfacial Colloidal Crystals, Phys Rev Lett, 1980, 45, 569572. 
[43] Dimitrov A S and Nagayama K, Continuous convective assembling of fine particles into twodimensional arrays on solid surfaces, Langmuir, 1996, 12, 5, 1303-1311.

[44] Rossi R. C, Tan M. X, and Lewis N. S, Size-dependent electrical behavior of spatially inhomogeneous barrier height regions on silicon, App Phy Lett, 2000, 77, 2698-2700.

[45] Hulteen J C, and Van Duyne R. Nanosphere lithography: A materials general fabrication process for periodic particle array surfaces. J. Vac. Sci. Tech. A, 1995, 13, 1553

[46] Deckman H. W and Dunsmuir J. H, Natural lithography, App Phy Lett, 1982, 41, 377-379

[47] Ruan W D, Lu Z C, Ji N, Wang C X, Zhao B, and Zhang J H, Facile fabrication of large area polystyrene colloidal crystal monolayer via surfactant-free langmuir-blodgett technique, Chem. Res Chinese U, 2007, 23, 712-714.

[48] Tabatabaei M, Sangar A, Kazemi-Zanjani N, Torchio P, Merlen A, Langugne- Labarthet F. Optical Properties of Silver and Gold Tetrahedral Nanopyramid Arrays Prepared by Nanosphere Lithography J. Phys. Chem. C 2013, 117, 14778-14786.

[49] Deckman, H. W., Dunsmuir, Natural lithography. J. H. Appl. Phys. Lett. 1982, 377.

[50] Bartlett P N, Birkin P R, Ghanem M A. Chem. Electrochemical deposition of microporous platinum, palladium, and cobalt films using polysytene latex sphere templates. Commun. 2000, 17, 1671-1672.

[51] Hulteen J C, Treichel D, Smith M, Duval M, Jenson T, Van-Duyne R. Nanosphere Lithography: Size-Tunable Silver Nanoparticle and Surface Cluster Arrays, J. Phys. Chem. B 1999, 103, 3854-3863

[52] Chou S Y, Krauss P R, Renstrom P J. Imprint of sub-25 nm via and trenches in polymers. Appl Phys Lett. 1995, 67, 3114.

[53] Chou S, Krauss P. Imprint Lithography with Sub-10nm Feature Size and High Throughput, Elsevier Science 1997, 35, 1-4, 237-240

[54] Hua F, Sun Y, Gaur A, et al. Polymer imprint lithography with molecular-scale resolution, Nano Letters 2004, 4, 2467-2471

[55] Lan H, Ding Y. Nanoimprint lithography, In: Lithography. Wang M, editor. Rijeka: InTech; 2010, 457-494.

[56] Colburn M, Johnson S, Stewart M, et al Step and flash imprint lithography: a new approach to high-resolution patterning, Proc. SPIE 1999, 3676, 379-389. 
[57] Malinovskis U, Poplausks R, Apsite I, et al. Ultrathin Anodic Aluminum Oxide Membranes for Production of Dense Sub-20 nm Nanoparticle Arrays, J. Phys. Chem. C 2014, 118, 8685-8690 [58] Su Z, Zhou W. Formation mechanism of porous anodic aluminium and titanium oxides, Adv Mater, 2008, 20, 3663-3667.

[59] Qiu T, Zhang W, Lang X, Zhou Y, Cui T, Chu P K. Controlled assembly of highly Ramanenhancing silver nanocap arrays templated by porous anodic alumina membranes. Small 20095 , 2333-7.

[60] Rabih O, Al-Kaysi, Tarek H. Ghaddar and Gonzalo Guirad. Fabrication of One-Dimensional Organic Nanostructures Using Anodic Aluminum Oxide Templates, J Nano. Mater, 2009, 436375, 14.

[61] Pimpin A, Srituravanich W, Review on Micro- and Nanolithography Techniques and Their Applications, Eng. J 2011, 16, 37-56.

[62] Madou M J. Fundamentals of Microfabrication: The Science of Miniaturization, 2nd ed. New York: CRC Press, 2002.

[63] Gates B D, Xu Q, Stewart M, Ryan D, Willson C G, Whitesides G M, New approaches to nanofabrication: Molding, printing, and other techniques, Chem. Rev. 2005 105, 1171-96.

[64] Altissimo M. E-beam lithography for micro/nanofabrication, Biomicrofluidics 2010, 4, 3-6.

[65] Liu H, Luo Y, Kong W et al. Large area deep subwavelength interference lithography with a $35 \mathrm{~nm}$ half-period based on bulk plasmon polaritons. Opt Mater Expr, 2018 8, 199-209.

[66] Whitney A V, Myers B D, Van Duyne R P. Sub-100 nm Triangular Nanopores Fabricated with the Reactive Ion Etching Variant of Nanosphere Lithography and Angle-Resolved Nanosphere Lithography, Nano Letters 2004, 4, 1507-1511.

[67] Popat K C, Mor G, Grimes C A, Desai T A. Surface modification of nanoporous alumina surfaces with poly ethylene glycol, Langmuir 2004, 20, 8035-8041

[68] Powell C J, Swan J B Effect of oxidation on the characteristic loss spectra of aluminium and magnesium. Phy Rev, 1960, 118, 640-643.

[69] Pines D, Bohm D. A Collective Description of Electron Interactions. I. Magnetic Interactions. Phy Rev, 1951, 82, 625-634.

[70] Pines D, Bohm D. A Collective Description of Electron Interactions: II. Collective vs Individual Particle Aspects of the Interactions. Phy Rev, 1952, 85, 338-353 
[71] Pines D, Bohm D. A Collective Description of Electron Interactions: III. Coulomb Interactions in a Degenerate Electron Gas. Phy Rev, 1953, 92, 609-626.

[72] Jeanmaire D L, Van Duyne R P. J. Surface Raman spectroelectrochemistry Part 1: Heterocyclic, aromtatic, and aliphatic amines absorbed on the anodized silver electrode, Electroanal. Chem., 1977, 8, 1-20.

[73] Albrecht M G. Creighton Anomalously intense Raman spectra of pyridine at a silver electrode. JA.J. Am. Chem. Soc.1977, 99, 5215-5127.

[74] Haes A J, Van Duyne R P. A Nanoscale Optical Biosensor: Sensitivity and Selectivity of an Approach Based on the Localized Surface Plasmon Resonance Spectroscopy of Triangular Silver Nanoparticles, J Am Chem Soc 2002, 124, 10596-10604.

[75] Hirsch L R, Jackson J B, Lee A, Halas N J, West J L. A Whole Blood Immunoassay Using Gold Nanoshells Anal Chem 2003, 75, 2377-2381.

[76] Sokolov K, Chumanov G, Cotton T. M. Enhancement of molecular fluorescence near the surface of colloidal metal films, Anal. Chem.,1998 70, 18, 3898-3905.

[77] Zeng J, Liang D, Cao Z. X. Applications of optical fiber SPR sensor for measuring of temperature and concentration of liquids, in Proc. SPIE, 2005, 5855, 667-670.

[78] Li M, Cushing S K, Wu N. Plasmon-enhanced optical sensors: a review, Analyst, 2015, 140, 386-406.

[79] Mie G. Beitrge zur optik tr"uber medien, speziell kolloidaler metal osungen, Annalen Der Physik, 1908, 330, 3, 377-445.

[80] Gans R. Uber die Form ultramikroskopischer Goldteilchen Ann. Phys, 1912, 37, 881-900.

[81] Camden J P, . Dieringer J A, Wang Y et al. Probing the Structure of Single-Molecule SurfaceEnhanced Raman Scattering Hot Spots, J. Am. Chem. Soc. 2008, 130, 12616-12617.

[82] Aravind P, Nitzan A, Metiu H. The Interaction Between Electromagnetic Resonances and its Role in Spectroscopic Studies of Molecules Adsorbed on Colloidal Particles or Metal Spheres, Surf. Sci., 1981, 110, 189-204.

[83] Jain P K, Huang W, El-Sayed W A. On the Universal Scaling Behavior of the Distance Decay of Plasmon Coupling in Metal Nanoparticle Pairs: A Plasmon Ruler Equation, Nano Lett. 2007, 7, 2080-2088. 
[84] Greeneltch N G, Blaber M G, Henry A, Schatz G C, Van Duyne R P. Immobilized Nanorod Assemblies: Fabrication and Understanding of Large Area Surface-Enhanced Raman Spectroscopy Substrates. Anal. Chem. 2013, 85, 2297-2303.

[85] Nahla A. Hatab, Chun-Hway Hsueh, Abigail L. Gaddis et al. Free-Standing Optical Gold Bowtie Nano antenna with Variable Gap Size for Enhanced Raman Spectroscopy, Nano Lett., 2010, 10, 4952-4955.

[86] Jung M, Kim J, Choi Y, Preparation of Anodic Aluminum Oxide Masks with Size-Controlled Pores for 2D Plasmonic Nanodot Arrays, J Nanomater. 2018. DOI:10.1155/2018/6249890.

[87] Wang T, Zhang J, Xue P et.al, Nanotransfer printing of gold disk, ring and crescent arrays and their IR range optical properties, J. Mater. Chem. C, 2014, 2, 2333-2340.

[88] Tabatabaei M, Sangar Q, Kazemi-Zanjani N, Torchio P, Merlen A, Lagugne-Labarthet F, Optical Properties of Silver and Gold Tetrahedral Nanopyramid Arrays Prepared by Nanosphere Lithography, J. Phys. Chem. C 2013, 117, 14778-14786.

[89] Xie W, Qiu P and Mao C. Bio-imaging, detection and analysis by using nanostructures as SERS substrates. J. Mater. Chem., 2011, 21, 5190-5202.

[90] Kahraman M, Mullen E R, Korkmaz A and Wachsmann-Hogiu S. Fundamentals and applications of SERS-based bioanalytical sensing. Nanophotonics 2017, 6, 831-852.

[91] Jeon T Y, Kim D J, Park S G, Ki S H, Kim D H. Nanostructured plasmonic substrates for use as SERS sensors. Nano Convergence, 2016, 3.

[92] Sharma B, Frotiera R, Henry A, Ringe R, Van Duyne R P. SERS: Mater App Futu. Mater Today, 2012, 5, 1-2.

[93] Marks H, Schechinger M, Garza J, Locke A and Cote G. Surface enhanced Raman spectroscopy (SERS) for in vitro diagnostic testing at the point of care. Nanophotonics 2017, 6, $681-701$.

[94] Li M, Cushing S K, Liang H, Suri S, Ma D, Wu N. Plasmonic Nanorice Antenna on Triangle Nano-array for Surface-enhanced Raman Scattering Detection of Hepatitis B Virus DNA. Anal. Chem, 2013, 85, 2072-2078.

[95] Zheng P, Kasani S. Shi X. et al. Detection of Nitrite with a Surface-Enhanced Raman Scattering Sensor Based on Silver Nanopyramid Array. Anal Chim Acta. 2018, 1040, 158-165. 
[96] Li M, Cushing S K, Zhang J et al. Three-Dimensional Hierarchical Plasmonic NanoArchitecture Enhanced Surface-Enhanced Raman Scattering Immunosensor for Cancer Biomarker Detection in Blood Plasma. ACS Nano. 2013, 7, 4967-4976.

[97] Zhang X, Dai Z, Si S et al. Ultrasensitive SERS Substrate Integrated with Uniform Subnanometer Scale "Hot Spots" Created by a Graphene Spacer for the Detection of Mercury Ions. Small 2017, 13, 1603347.

[98] Zhao Y, Zhao S, Zhang L, Liu Y, Li X, Lu Y. A three-dimensional Au nanoparticlemonolayer graphene-Ag hexagon nano-array structure for high-performance surface-enhanced Raman scattering. RSC Adv., 2017, 7, 11904-11912.

[99] Gao X, Zheng P, Kasani S et al. Paper-Based Surface-Enhanced Raman Scattering Lateral Flow Strip for Detection of Neuron-Specific Enolase in Blood Plasma. Anal. Chem. 2017, 89, 10104-10110.

[100] Li M, Cushing S K, Zhang J et al. Shape-dependent surface-enhanced Raman scattering in gold-Raman-probe-silica sandwiched nanoparticles for biocompatible applications. Nanotechnology 2012, 23, 115501-115511.

[101] Park J E, Lee Y, Nam J. M. Precisely Shaped, Uniformly Formed Gold Nanocubes with Ultrahigh Reproducibility in Single-Particle Scattering and Surface-Enhanced Raman Scattering. Nano Lett. 2018, 18, 6475-6482

[102] Scarabelli L, Coronado-Puchau M, Giner-Casares J J, Langer J, and Liz-Marza' L M. Monodisperse Gold Nanotriangles: Size Control, Large-Scale Self- Assembly, and Performance in Surface-Enhanced Raman Scattering. ACS Nano 2014, 8,6, 5833-5842.

[103] Chen S, Liu D, Wang Z, Sun X, Cui D, Chen X. Picomolar detection of mercuric ions y means of gold silver core shell nanorods. Nanoscale 2013, 5, 6731-6735.

[104] Agranovich V M, Mills D L. Surface Polaritons: Electromagnetic Waves at Surfaces and Interfaces, North-Holland, Amsterdam, 1982.

[105] Otto. A. Spectra of Plasmon Polaritons at Metal-Insulator Interfaces of a Nanosized Gold Film: Expansion into Components and Their Systematization. Z. Phys. 1968, 216, 398-410.

[106] Kretschmann E, Raether H. Radiative Decay of Nonradiative Surface Plasmons Excited by Light Z. Nature A 1968, 23, 2135-2136. 
[107] Barnes W L, Dereux A, and Ebbesen T W. Surface plasmon subwavelength optics, Nature 2003, 424, 824-830.

[108] Lackowicz J R. Plasmonics in Biology and Plasmon-Controlled Fluorescence. Plasmonics, 2006, 1, 5-33

[109] Dostalek J, Knoll W. Biosensors based on surface plasmon-enhanced fluorescence spectroscopy. Biointerphases, 2008, 3, 12-22.

[110] Zhang J, Zhang L, Xu W. Surface plasmon polaritons: physics and applications. J. Phys. D: Appl. Phys. 2012, 45, 113001

[111] Ebbesen T W, Lezec H, Ghaemi H F, Thio T, and Wolff P A. Extraordinary optical transmission throughsub-wavelength hole arrays. Nature, 1998, 391, 667-669.

[112] Fang Y, Sun M. Nanoplasmonic waveguides: towards applications in integrated nanophotonic circuits. Light. Sci App 2005, 4, 294.

[113] Fleischmann M, PJ Hendra, AJ McQuillan. Raman Spectra of Pyridine Adsorbed at a Silver Electrode. Chem Phy Lett. 1974, 26, 163-166.

[114] Zheng P, Cushing S K, Suri S, Wu N. Phys. Chem. Chem. Phys., 2015, 17, 21211-21219

[115] Kalachyova Y, Mares D, Lyutakov O, Kostejn M, Lapcak L, and Svorcik V. Surface Plasmon Polaritons on Silver Gratings for Optimal SERS Response. J. Phys. Chem. C, 2015, 119, 9506-9512.

[116] Haynes C L, McFarland A D, van Duyne R P. Surface-enhanced: raman spectroscopy. Anal Chem, 2005, 77, 338-346.

[117] Zijlstra P, Chon J W M, Gu M. Five-dimensional optical recording mediated by surface plasmons in gold nanorods. Nature 2009, 459, 410-413

[118] Grubisha D S, Lipert R, Park H Y, Driskill J, Porter M. Anal Chem 2003 75, 21, 5936-5943. [119] Yun L, Qiang L, Shimeng C, Fang C, Hanqi W and Wei P. Surface plasmon resonance biosensor based on smart phone platforms, Sci Rep, 2015, 5, 12864.

[120] Liang Y, Zhang S, Cao Y, Lu Y, Xu T. Free-standing plasmonic metal-dielectric-metal bandpass filter with high transmission efficiency. Sci Rep, 2017, 7, 4357

[121] Brolo A G, Gordon R, Leathem B, Kavanagh K L. Surface plasmon sensor based on the enhanced light transmission through arrays of nanoholes in gold films. Langmuir 2004, 20, 48134815. 
[122] Valsecchi C, Armas L. E. G., Menezes J.W. Large Area Nanohole Arrays for Sensing Fabricated by Interference Lithography, Sensors, 2019, 19 (9): 2182.

[123] Lin L. Manipulation of Near Field Propagation and Far Field Radiation of Surface Plasmon Polariton, Springer Singapore 2017.

[124] Frederich H, Wen F, Laverdant J. et al. Determination of the Surface Plasmon Polariton Extraction Efficiency from a Self-Assembled Plasmonic Crystal. Plasmonics (2014) 9: 917.

[125] Dhawan A, Canva M, Vo-Dinh T. Narrow groove plasmonic nano-gratings for surface plasmon resonance sensing, Opt. Express 2011, 19, 787-81.

[126] Kalachyova Y, Mares D, Lyutakov O, Kostejn M, Lapcak L, vorcík V Š. Surface Plasmon Polaritons on Silver Gratings for Optimal SERS Response. J. Phys. Chem. C 2015, 119, 9506-9512.

[127] Xiao C, Chen Z, Qin M, Zhang D and Fan L. Composite Sinusoidal Nanograting With LongRange SERS Effect for Label-Free TNT Detection. Photonic Sensors 2018, 8, 278-288.

[128] Chu Y, Banaee M G, Crozier K B Double-Resonance Plasmon Substrates for SurfaceEnhanced Raman Scattering with Enhancement at Excitation and Stokes Frequencies. ACS Nano. 4, 2804-2810.

[129]. Du L, Zhang X, Mei T, Yuan X. Localized surface plasmons, surface plasmon polaritons, and their coupling in 2D metallic array for SERS. Optics Express. 2010, 18, 1959-1965.

[130] Wang J, Lin W, Cao E, Xu X, Liang W, and Zhan X.. Surface Plasmon Resonance Sensors on Raman and Fluorescence Spectroscopy. Sensors 2017, 17, 2719.

[131] Fano U. Effects of Configuration Interaction on Intensities and Phase Shifts. Phys. Rev. 1961, 124, 1866-1878.

[132] Limonov M F, Rybin M V, Poddubny A N and Kivshar Y S, Fano resonances in photonics. Nat Photo, 2017, 11, 543-554.

[133] Luk'yanchuk B, Zheludev N I, Maier S A et al. The Fano resonance in plasmonic nanostructures and metamaterials. Nat Mater 2010, 9, 707-715.

[134] Miroschnichenko A, Flach S, Kivshar Y S. Fano resonances in nanoscale structures. Rev. Mod. Phys., 2010, 82, 3, 2257-2298.

[135] Ho C C, Zhao K, Lee T Y. Quasi-3D gold nanoring cavity arrays with high-density hot-spots for SERS applications via nanosphere lithography. Nanoscale, 2014, 6, 8606-8611. 
[136] Fan J A, Wu C, Bao K et al. Self-assembled plasmonic nanoparticle clusters. R. Science 2010, 328, 1135-1138.

[137] Neubrech F, Pucci A, Cornelius T. W, Karim S, García- Etxarri, A, Aizpurua. Resonant plasmonic and vibrational coupling in a tailored manoantenna for infrared detection. J. Phys. Rev. Lett. 2008, 101, 157403-157407.

[138] Shapiro M. Electromagnetically Induced Transparency with Structured Multicontinua. Phys. Rev. A 2007, 75, 013424-013433.

[139] Neubrech F, Weber D, Enders D, Nagao T, Pucci A. Antenna Sensing of Surface Phonon Polaritons. J. Phys. Chem. C 2010, 114, 7299-7301.

[140] Gallinet B, Lovera A, Siegfried T, Sigg H, Martin O J F. Fano resonant plasmonic systems: Functioning principles and applications. AIP Conf. Proc. 2012, 1475, 18-20.

[141] Fano U. The theory of anomalous diffraction grating and of quasi-stationary waves on metallic surfaces ( Sommerfeld's waves). J. Opt. Soc. Am. 1941, 31, 213-222.

[142] Hentschel M, Saliba M, Vogelgesang R, Giessen H, Alivisatos A P, Liu N. Transition from Isolated to Collective Modes in Plasmonic Oligomers. Nano Lett. 2010, 10, 2721-2726.

[143] Zhao W, Jiang H, Liu B, Jiang Y, Tang C, Li J. Fano resonance based optical modulator reaching 85\% modulation depth. Appl. Phys. Lett. 2015, 107, 171109.

[144] Zhao W, Ju D, Jiang Y, Zhan Q. Dipole and quadrupole trapped modes within bi-periodic Silicon particle array realizing three-channel refractive sensing. Opt Exp, 2014, 22, 31277-31285. [145] Ajuan Cui, Zhe Liu, Jiafang Li et al. Directly patterned substrate-free plasmonic "nanograter" structures with unusual Fano resonances. Light: Science \& Applications 2015, 4,308

[146] Zhan S, Peng Y, He Z et al. Tunable nanoplasmonic sensor based on the asymmetric degree of Fano resonance in MDM waveguide. Scie Rep (2016), 22428, 6.

[147] Huang T, Zeng S, Zhao X, Cheng Z, Shum P P. Fano Resonance Enhanced Surface Plasmon Resonance Sensors Operating in Near-Infrared. Photonics, 2018, 5, 23.

[148] Chen J, Gan F, Wang Y, Li G. Plasmonic Sensing and Modulation Based on Fano Resonances. Adv. Optical Mater. 2018, 6, 1701152. 
[149] Zhang S, Bao K, Halas N, Xu H, Nordlnader P. Substrate Induced Fano Resonances of a Plasmonic Nanosube: A Route to Increased Sensitivity Localized Surface Plasmon Resonance Sensors Revealed. Nano Letter. 2011, 11, 1657-1663.

[150] Cetin A E, Altug H. Fano Resonant Ring/Disk Plasmonic Nanocavities on Conducting Substrates for Advanced Biosensing. ACS Nano 2012, 6, 9989-9995.

[151] Deng H, Chen X, Xu Y, Miroshnichenko A.E. Single protein sensing with asymmetric plasmonic hexamer via Fano resonance enhanced two-photon luminescence. Nanoscale 2015, 7, 20405-20413.

[152] Yanik A A, Cetin A E, Huang M. Seeing protein monolayers with naked eye through plasmonic Fano resonances. PNAS 2011, 108, 11784-11789.

[153] Féron P. Whispering Gallery Mode Lasers in Erbium doped fluoride glasses. Annales de la Fondation Louis de Broglie 2004, 29, 317-329.

[154] Vollmer F, Arnold S. Whispering-gallery mode biosensing: label free detection down to the single molecules. Nat Meth, 2008, 5, 591-596.

[155] Vollmer F, Arnold S, Keng D.. Single virus detection from the reactive shift of a whisperinggallery mode. PNAS 2008, 105, 20701-20704.

[156] Ausman L K, and Schatz G C. Whispering-gallery mode resonators: Surface enhanced Raman scattering without plasmons. J. Chem. Phys. 2008, 129, 054704

[157] Fan H, Xia C, Fan L, Wang L, Shen M. Graphene supported plasmonic whispering-gallery mode in a metal-coated microcavity for sensing application with ultrahigh sensitivity. Optics communications 2018, 410, 668-673.

[158] Kang T Y, Lee W, Ahn H. et al. Plasmon coupled whispering gallery modes on nanodisk arrays for signal enhancements. Sci Rep 2017, 7, 11737.

[159] Schweinsberg A, Hodce S, Lepeshkin N, Boyd R, Chase C, Fajardo J. An environmental sensor based on an integrated optical whispering gallery mode risk resonator. Sensor Actuat, 2007, $123,727-732$.

[160] Li C, Teimourpoura M H, McLeod E, Sua J. Enhanced whispering gallery mode sensors. Proc. of SPIE 2018, 10629, 201.

[161] Foreman M R, Swaim J D, Vollmer F. Whispering gallery mode sensors, Adv Optic Photo, 2015, 7, 168-240. 
[162] Min B, Ostby E, Sorger V. High-Q surface-plasmon-polariton whispering-gallery microcavity. Nature 2009, 457, 455-458.

[163] Arnold S, Dantham V R, Barbre C, Garetz B A, Fan X. Periodic plasmonic enhancing epitopes on a whispering gallery mode biosensor. Opt. Express 2012, 20, 26147-26159

[164] Vesseur E J R, Garc1'a de Abajo F J and Polman A, Modal Decomposition of SurfacePlasmon Whispering Gallery Resonators. Nano Lett. 2009, 9, 3147-3150.

[165] Dantham V R, Holler S, Barbre C, Keng D, Kolchenko V, and Arnold S. Label-free detection of single protein using a nanoplasmonic photonic hybrid microcavity. Nano Lett. 2013, 13, 33473351.

[166] Bozzola A, Perotto S, De Angelis F. Hybrid plasmonic-photonic whispering gallery mode resonators for sensing: a critical review. Analyst, 2017, 142, 883-898.

[167] Vahala K J. Optical microcavities. Nature 2003, 424, 839-846

[168] Noda S, Fujita M, Asano T. Spontaneous-emission control by photonic crystals and nanocavities. Nature Photon 2007, 1, 449-458.

[169] Kravets V G, Kabashin A V, Barnes W L, Grigorenko A N. Plasmonic Surface Lattice Resonances: A Review of Properties and Applications. Chem. Rev. 2018, 118, 5912-5951.

[170] Rajeeva B B, Lin L, Zheng Y. Design and applications of lattice plasmon resonances. Nano Research 2018, 11, 4423-4440.

[171] Humphrey A D, Barnes W L. Plasmonic Surface Lattice Resonances on Arrays of Different Lattice Symmetry. Phys. Rev. B: Condens. Matter Mater. Phys. 2014, 90, 075404-075412.

[172] Haynes C.L, McFarland A D, Zhao L L et al. Nanoparticle optics: The importance of radiative dipole coupling in two-dimensional nanoparticle arrays. J. Phys. Chem. B 2003, 107, 7337-7342.

[173] Humphrey A, Meinzer N, Starkey R A, Barnes W L. Surface Lattice Resonances in Plasmonic Arrays of Asymmetric Disc Dimers. ACS Photonics 2016, 3, 634-639.

[174] Zhukovsky S V, Babicheva V E, Uskov A V, Protsenko I E, Lavrinenko A V. Enhanced Electron Photoemission by Collective Lattice Resonances in Plasmonic Nanoparticle-Array Photodetectors and Solar Cells. Plasmonics 2014, 9, 283-289.

[175] Lin L, Zheng Y. Optimizing Plasmonic Nanoantennas Via Coordinated Multiple Coupling. Sci. Rep. 2015, 5, 14788. 
[176] Ng C, Dligatch S, Amekura H, Davis T J, Goḿez D E. Waveguide-Plasmon Polariton Enhanced Photochemistry. Adv. Opt. Mater. 2015, 3, 1582-1590.

[177] Sadeghi S M, Wing W J, Campbell Q. Tunable plasmonic-lattice mode sensors with ultrahigh sensitivities and figures of merits. J. Appl. Phys. 2016, 119, 244503-5.

[178] Gutha R, Sadeghi S, Sharp C, Wing W J. Biological sensing using hybridization phase of plasmonic resonances with photonic lattice modes in arrays of gold nanoantennas, Nanotechnology 2017, 28, 355504-355511.

[179] Kuznetsov A I, Evlyukhin A B, Goncalves M. R et al. Laser fabrication of large-scale nanoparticle arrays for sensing applications. ACS Nano 2011, 5, 4843-4849.

[180] Shen Y, Zhou J. H, Liu T. R et al. Plasmonic gold mushroom arrays with refractive index sensing figures of merit approaching the theoretical limit. Nat. Commun. 2013, 4, 2381.

[181] Lin L H, Zheng Y B. Optimizing plasmonic nanoantennas via coordinated multiple coupling. Sci. Rep. 2015, 5, 14788.

[182] Li Z Y, Butun S, Aydin K. Ultranarrow band absorbers based on surface lattice resonances in nanostructured metal surfaces. ACS Nano 2014, 8, 8242-8248.

[183] Bahramipanah M, Dutta-Gupta S, Abasahl B, Martin O J F. Cavity-coupled plasmonic device with enhanced sensitivity and figure-of-merit. ACS Nano 2015, 9, 7621-7633.

[184] Lin L H, Zheng Y B. Engineering of parallel plasmonic- photonic interactions for on-chip refractive index sensors. Nanoscale 2015, 7, 12205-12214.

[185] Cesario J, Quidant R, Badenes G, Enoch S. Electromagnetic coupling between a metal nanoparticle grating and a metallic surface. Opt. Lett. 2005, 30, 3404-3406.

[186] Norlander P, Le F. Plasmonic structure and electromagnetic field enhancements in the metallic nanoparticle-film system, Appl. Phys. B 2006, 84, 35-41.

[187] Zheng P, Kasani S, Wu N. Converting plasmonic light scattering to confined light absorption and creating plexcitons by coupling a gold nano-pyramid array onto a silica-gold film, Nanoscale Horiz. 2019, Advance Article.

[188] Bozhevolnyi S I, Effective-index modeling of channel plasmon polaritons. Opt. Express 2006, 14, 9467-9476.

[189] Søndergaard T, Bozhevolnyi S. Slow- Plasmon resonant nanostructures scattering and field enhancements Phys. Rev. B 2007, 75, 073402-073408. 
[190] Fang Z, Zhen Y R, Fan L, Zhu X, Nordlander P. Tunable wide-angle plasmonic perfect absorber at visible frequencies, Physical Review B 2012, 85, 245401.

[191] Dutta A, Naldoni A, Malara F, Govorov A O, Shalaev V, Boltasseva A. Gap-plasmon enhanced water splitting with ultrathin hematite films: The role of plasmonic-based light trapping and hot electrons. Faraday Discuss. 2019, Advance Article.

[192] Fofang N T, Grady N K, Fan Z, Govorov A O, and Halas N J, Plexciton Dynamics: ExcitonPlasmon Coupling in a J-Aggregate Au Nanoshell Complex Provides a Mechanism for Nonlinearity, Nano Lett. 2011, 11, 1556-1560.

[193] Chang S W, Lin T R, Chuang S L. Theory of plasmonic Fabry-Perot nanolasers. Opt. Express 2010, 18, 15039-15053. 


\section{CHAPTER 3: FABRICATION AND OPTICAL PROPERTIES OF LARGE-AREA PLASMONIC GOLD NANO RING ARRAY PATTERN}

A new fabrication route, which combines the nanosphere lithography with the siliconbased clean-room microfabrication processes, has been developed to produce the large-area longrange ordered gold nanoring array patterns in a controllable fashion. Both the experimentation and the finite-difference time-domain (FDTD) simulation show that the surface plasmon resonance peak (SPR) of the nanoring array pattern can be tuned systematically in a large spectral range by varying the geometry parameters such as the ring thickness, the ring height, the ringer outer diameter and the gap between neighboring rings. For the Au nanoring arrays with a large gap in the absence of plasmon coupling between neighboring rings, the local electromagnetic (EM) field enhancement occurs at both the outer and inner surfaces of individual nanorings; and the periodicity of Au nanoring array has no effect on the plasmonic properties. For the Au nanoring arrays with a small gap, plasmon coupling takes place between neighboring rings. As a result, the characteristic plasmonic band is split into two new peaks corresponding to a bonding SPR mode and an antibonding SPR mode. The local EM field enhancement becomes stronger with a decrease in the gap between neighboring rings, but the SPR peaks shift away. Therefore, to maximize the surface-enhanced Raman scattering signal, the geometry parameters of the Au nanoring array need to be tuned to balance the contributions from the resonance excitation (spectral overlap) and the local EM field enhancement. In this work, miRNA detection is demonstrated using a novel method of fabricating Nano ring array using Nanosphere Lithography (NSL), a long range periodic and economical technique of nanostructure fabrication. The nano ring array optical properties are investigated and FDTD simulations are performed to correlate structural changes with optical response and electromagnetic field. The ring structure height, radius thickness and pitch are optimized to analyze the SERS enhancement at $785 \mathrm{~nm}$ excitation laser. Nano ring array is engineered in such a way each ring generates 6 hotspots from coupling from the surrounded ring structures with nano gaps less than 10nm, allowing high SERS enhancement for detection of miRNA in human fluids. 


\subsection{INTRODUCTION}

Surface-enhanced Raman scattering (SERS) and surface plasmon-coupled emission (SPCE) spectroscopy are finding increasing applications in biosensing and bioimaging. ${ }^{1-5}$ In SERS and SPCE, plasmonic nanostructures are employed as substrates to amplify the SERS or SPCE signals. Plasmonic metal nanoparticles have been used extensively in SERS and SPCE. Bare metal nanoparticles tend to aggregate in a solution with high ionic strength. Hence surface modification of metal nanoparticles is often performed to preserve the stability of colloidal nanoparticles. ${ }^{6}$ Besides free-standing nanoparticles, two-dimensional (2D) metal nanoarray patterns have received increasing attention. ${ }^{7-10}$ The optical properties of 2D nanoarray patterns can be tuned systematically, and predicted theoretically using numeric methods such as the finite-difference time-domain (FDTD) and the finite element analysis (FEA), which in return helps guide the design of 2D nanoarrays. ${ }^{11-12}$ Long-range ordered 2D nanoarray patterns not only show good reproducibility but also extend the dynamic detection range of sensors due to the extension of enhanced electromagnetic (EM) field in space. It still remains a challenge in massive production of large-area long-range ordered metal nanoarray patterns at low cost.

Among the panoply of 2D metallic nanoarrays, Au nanoring arrays display meritorious features. ${ }^{13-19}$ First, Au nanoring array patterns have a wide spectral range of tunable plasmon, which have advantages over Au nanohole and nanoparticle arrays whose optical property tunning is severely limited by the extent of size-dependent plasmon shift. Second, the EM field can be concentrated at both the inner and outer surfaces of the ring, considerably extending the spatial distribution of "hot spots" for surface-enhanced spectroscopies. Third, the overall size of $\mathrm{Au}$ nanoring arrays can remain largely unchanged during optical tuning by simply changing the shell thickness of rings. In contrast, tailoring optical properties of Au nanohole and nanoparticle arrays is always at the expense of significantly varying the size or periodicity, which compromises the overall performance. In addition, Au nanoring arrays have been theoretically predicted to have a high orientation-independent plasmon resonant absorption in the visible to near-infrared spectrum, which have strong implications for applications in plasmon-enhanced photothermal heating. ${ }^{20}$ Thanks to these advantageous properties, colloidal Au nanorings have been chemically synthesized using sacrificial Co nanoparticles for SERS applications. ${ }^{21-22}$ These Au colloidal Au nanorings obtained have an outer diameter from 25 to $40 \mathrm{~nm}$ with a shell thickness of $5 \mathrm{~nm}$, which 
allows the LSPR to be tuned from 651 to $760 \mathrm{~nm}$. Au nanoring array patterns have been fabricated by nanosphere lithography (NSL), electron-beam and focused-ion beam lithography, and etc.. ${ }^{13-14 \text {, }}$ ${ }^{17-19}$ Electron-beam and focused-ion beam lithography can create ultra-small features in accurate and controllable fashion, but is expensive and impossible to produce the nano-array patterns massively. For example, Au nanoring arrays which were fabricated by e-beam lithography to study substrate effects on the behavior of plasmons only have an area of $60 \mu \mathrm{m} \times 60 \mu \mathrm{m} .{ }^{23}$

(a) Si substrate cleaning

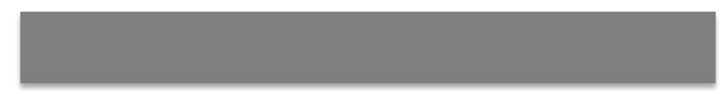

(b) PS beads patterning

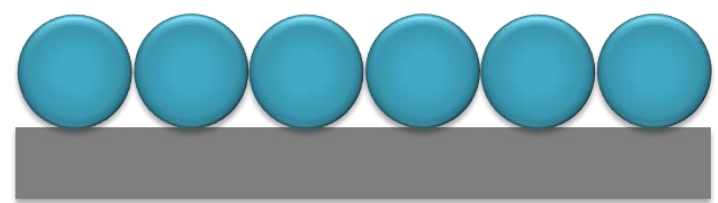

(c) PS beads etching

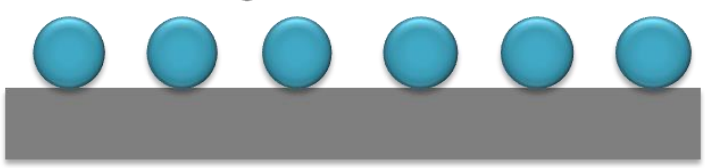

(d) Si etching with PS beads as a mask

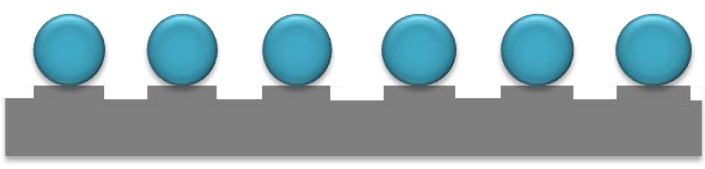

(e) PS beads etching

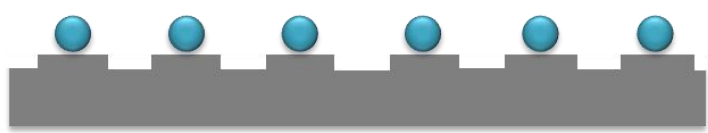

(f) Ni deposition

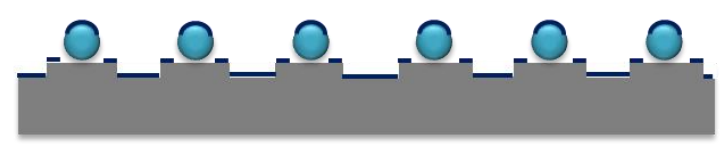

(g) PS beads removal

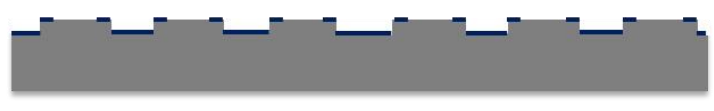

(h) Si etching with Ni layer as a mask

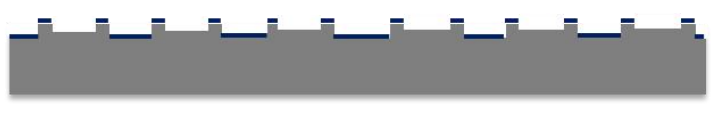

(i) Ni layer removal

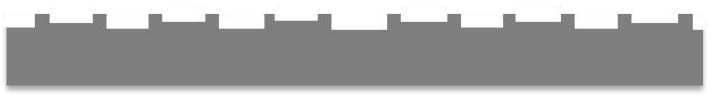

(j) Au deposition

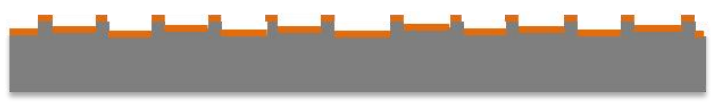

(k) Schematic Au nano-ring array

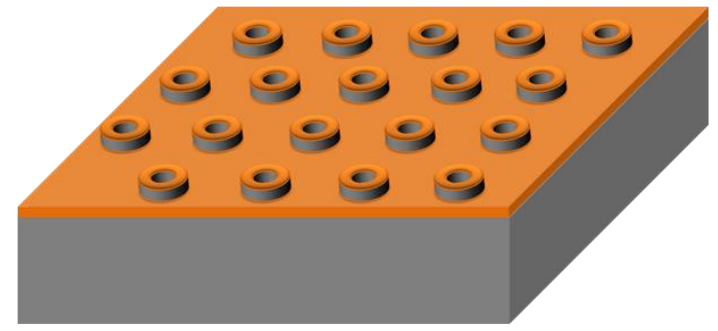

Figure 3. 1: Fabrication protocol for Au nanoring arrays

In contrast, NSL is a powerful, versatile, and cost-effective fabrication method for fabrication of a large-area periodic nano-array patterns. NSL uses polystyrene (PS) beads as the template, which can be easily tailored by simply changing the PS bead size, the etching time, and 
the metal deposition thickness. The reliability of NSL has been manifested in the development of Au nanohole arrays and Au nanotriangle arrays for SERS and SPCE applications. ${ }^{7,24-25}$ Among those $\mathrm{Au}$ nanoring array patterns reported previously, the plasmonic bands of most of them are located in the mid-infrared and near-infrared range (typically >900 nm). This does not meet the requirement for SERS sensing because most of current SERS sensing signals are excited by the $785 \mathrm{~nm}, 633 \mathrm{~nm}$ and $532 \mathrm{~nm}$ lasers. Although NSL has been demonstrated to fabricate Au nanoring arrays in combination with electrochemical deposition, the nanoring structure obtained is irregular, restricting achieving the best possible performance. ${ }^{26}$ Also, the gap of nanorings is generally too large to have plasmonic coupling between neighboring rings, leading to no "hot spots" between neighboring rings. In addition, so far a few studies have been performed to systematically investigate the EM enhancement distribution and the optical properties of nanoring array patterns.

In the present work, NSL is combined with the silicon-based clean-room fabrication processes to fabricate the large-area long-range ordered Au nanoarray patterns at low cost. FDTD simulation is employed to predict the optical properties and the EM field distribution in the Au nanoarray patterns with systematical variation of the geometry parameters such as the periodicity, the ring thickness, height, and the overall size of the rings. The systematic FDTD studies on the optical properties will provide guidelines for designing the optimum plasmonic Au nanoring array pattern for SERS sensing. The Au nanoring array patterns investigated in this work show two unique features for SERS. First, the wide tunable spectral range plasmon across the visible-light and near-infrared light regions to enable the plasmon band match the typical spectral wavelength of excitation laser sources. Second, the local EM field enhancement at the gap of neighboring rings provides a strong near-field.

\subsection{EXPERIMENTAL METHODS}

\section{Chemicals and reagents}

Silicon wafers were purchased from University Wafers Inc. (Boston, MA). Polystyrene microspheres with diameters of $500 \mathrm{~nm}, 600 \mathrm{~nm}$, and $1000 \mathrm{~nm}$ were purchased from Thermo Scientific (Waltham, MA). Deionized (D.I.) water was produced by Milli-Q Integral 3/5/10/15 system (18.2 MO cm, Millipore Corp., USA). 


\section{Instrumentation}

Oxygen Plasma Asher (March PX-250 Plasma Asher) was used to etch the PS beads. Inductively Coupled Plasma (ICP Trion Technology Minilock III RIE) was used to etch silicon. E-beam Evaporator (Kurt J. Lesker) was used for metal deposition. A Scanning Electron Microscope (JEOL-JSM-7600F) was used to characterize the Au nanoring array patterns. Reflection spectra of Au nanoring array patterns were acquired using an Ocean Optics USB 4000 spectrometer and DTMINI-2-GS.

\section{Finite-difference time-domain (FDTD) simulation}

Optical properties of Au nanoring arrays were studied using FDTD simulations. The software Optiwave FDTD is commercially available from Optiwave Inc. A grid size of $1 \mathrm{~nm}$ was used to construct the simulation cells. A continuous plane wave from $400 \mathrm{~nm}$ to $1200 \mathrm{~nm}$ was used as the input light source. The refractive index was taken from the data of Palik.27 Periodic boundary conditions were imposed for all simulations to replicate periodic Au nanoring array patterns.

\subsection{RESULTS and DISCUSSIONS}

\section{Morphology of Au nanoring array patterns}

Figure 3.2(a) shows that the fabricated Au nanoring array pattern had a typical physical size comparable with a coin. Figures 3.2(b) to 3.2(f) show the SEM images of the Au nanoring array patterns fabricated using the PS spheres with different sizes. In Figure 3.2(b), the overall ring size (i.e., the outer ring radius) was $920 \mathrm{~nm}$, the ring thickness was $75 \mathrm{~nm}$, the ring height was 120 $\mathrm{nm}$, and the periodicity (center-to-center distance) was $1000 \mathrm{~nm}$. The periodicity of Au nanoring arrays is determined by the size of unetched PS beads used. The overall ring size and the gap size, which is the closest distance between two neighboring rings, can be varied by changing the duration of sphere etching Figure 3.1(c); and the ring height is dependent on the Si etching. The ring thickness, which is defined as the difference between outer and inner ring radius, can be tuned by controlling the oxygen plasma etching duration in Figure 3.1(e). While the hexagonally patterned monolayer of PS beads renders a long-range order of Au nanoring array pattern as shown in Figure 3.2(e) and 3.2(f). The parameters such as the size of PS beads and the etching duration allow a high degree of tunability of the optical properties. 


\section{Optical properties of Au nanoring array patterns}

The optical properties of Au nanoring arrays can be described by the plasmon hybridization model. ${ }^{283}$ As an Au nanoring array is considered to be created by an Au nanodisk array perforated with an Au nanohole array. Hence its optical properties include the contributions from both the elements. The elementary nanodisk and nanohole plasmons contribute to the oscillating charges at both the outer and inner surface of the ring. Because of a finite ring thickness, these oscillating charges interact with each other and produce two new plasmon resonances corresponding to a bonding mode at a longer wavelength and an antibonding mode at a shorter wavelength. The bonding localized surface plasmon resonance (LSPR) mode of an Au nanoring array is identified as the reflection dip at a spectral wavelength of $750 \mathrm{~nm}$ for the Au nanoring array with a periodicity of $500 \mathrm{~nm}$ (Figure 3(a)); and the dip red-shifted to a spectral wavelength of $880 \mathrm{~nm}$ when the Au ring periodicity increased to $600 \mathrm{~nm}$. Due to the cancellation of opposite charges at outer and inner surface of the ring for the antibonding mode, the corresponding reflection dip has a much lower intensity and is thus of less significance.
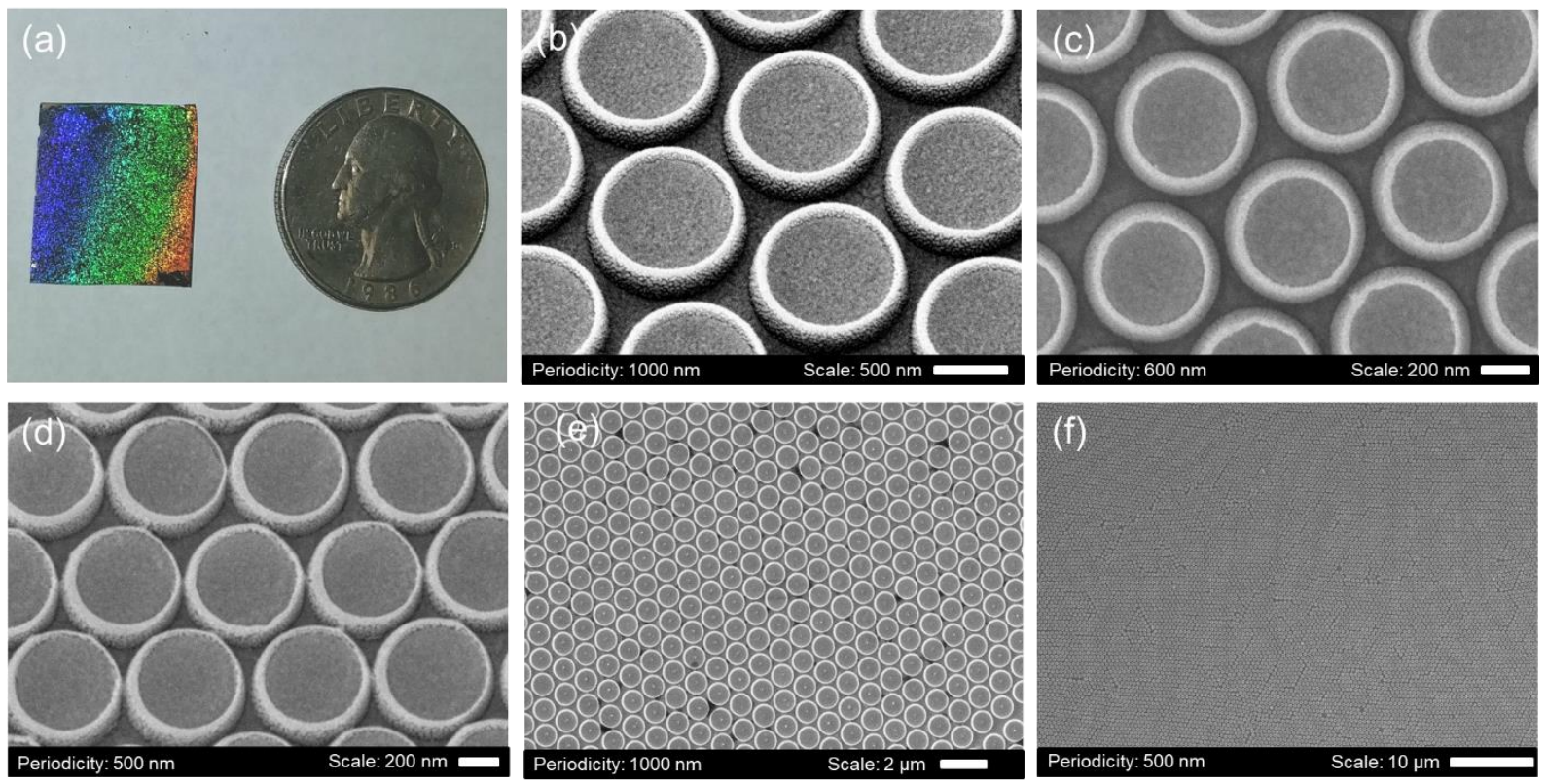

Figure 3.2. (a) Optical image of a large-area Au nanoring array chip compared to a coin; SEM image of $(b)$ the Au nanoring pattern (periodicity:1000 nm, outer radius: $920 \mathrm{~nm}$, thickness: 75 $\mathrm{nm}$ and height: $120 \mathrm{~nm}$ ); (c) Pattern (periodicity: $600 \mathrm{~nm}$, outer radius: $500 \mathrm{~nm}$, thickness: $50 \mathrm{~nm}$ 
and height: $120 \mathrm{~nm}$ ); (d) pattern (periodicity: $500 \mathrm{~nm}$, outer radius: $440 \mathrm{~nm}$, thickness: $30 \mathrm{~nm}$ and height: $120 \mathrm{~nm}) ;(e)$ and (f) the Au nanoring array patterns with large scale area.

The plasmon hybridization description of the Au nanoring arrays with periodicity of 500 $\mathrm{nm}$ and $600 \mathrm{~nm}$ predicted by FDTD simulation was consistent with the experimental data of reflection spectra periodicities Figure 3.3(a). The intense bonding LSPR mode was further confirmed by the dipolar behavior observed in the simulated local EM field distribution extracted at the LSPR wavelength, as shown in Figure 3.3(b). The Au nanoring array acts as a plasmonic lens. It concentrates the EM radiation at both outer and inner surface of the ring, considerably extending the spatial distribution of "hot spots" for surface-enhanced spectroscopies. In comparison, "hot spots" are limited to outer surface of circular nanodiscs.
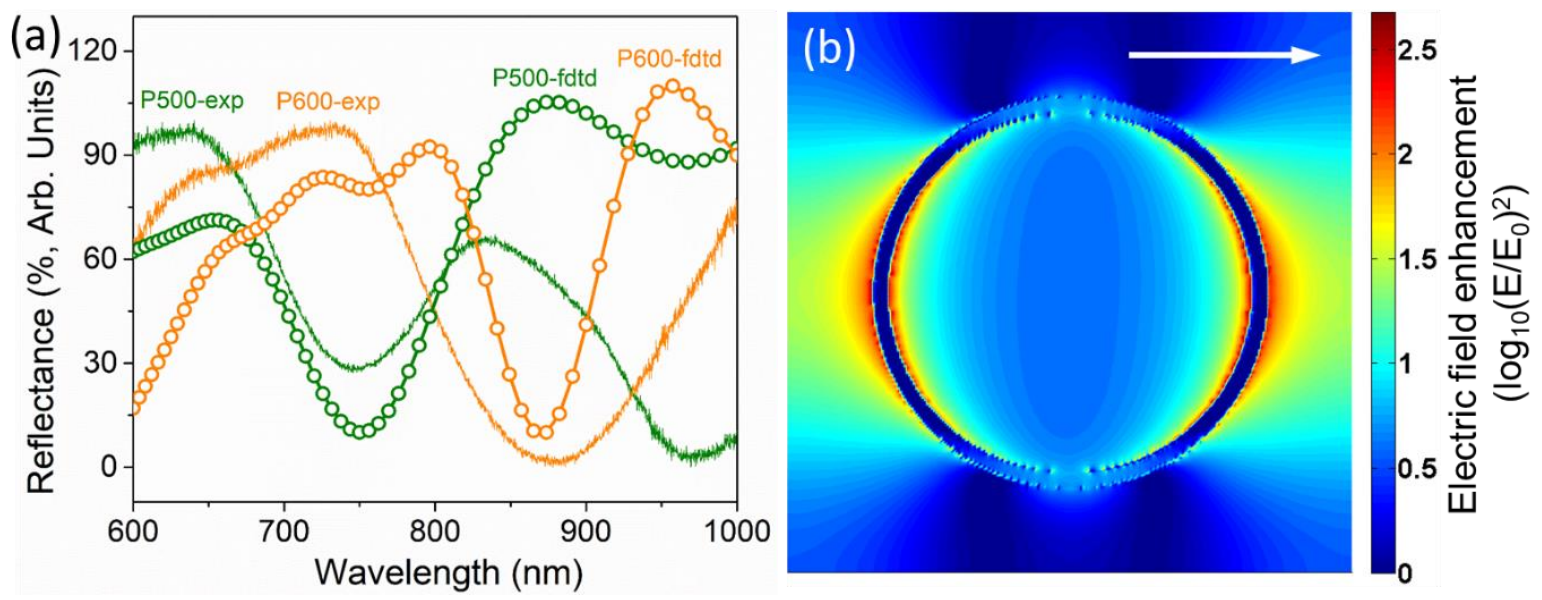

Figure 3.3. (a) Experimental (P500-exp) and FDTD-simulated (P500-fdtd) reflection spectra of Au nanoring arrays with periodicity of $500 \mathrm{~nm}$ and $600 \mathrm{~nm}$, respectively; (b) EM field distribution with a local EM field enhancement $\left(E / E_{0}\right)^{2}$ of $\sim 5.0 \times 10^{2}$ at the plasmon peak wavelength for an $A u$ nanoring with an outer radius of $200 \mathrm{~nm}$, a ring thickness of $40 \mathrm{~nm}$, a height of $100 \mathrm{~nm}$ and a periodicity of $500 \mathrm{~nm}$. The white arrow indicates the polarization direction of the incident light. 
(a)
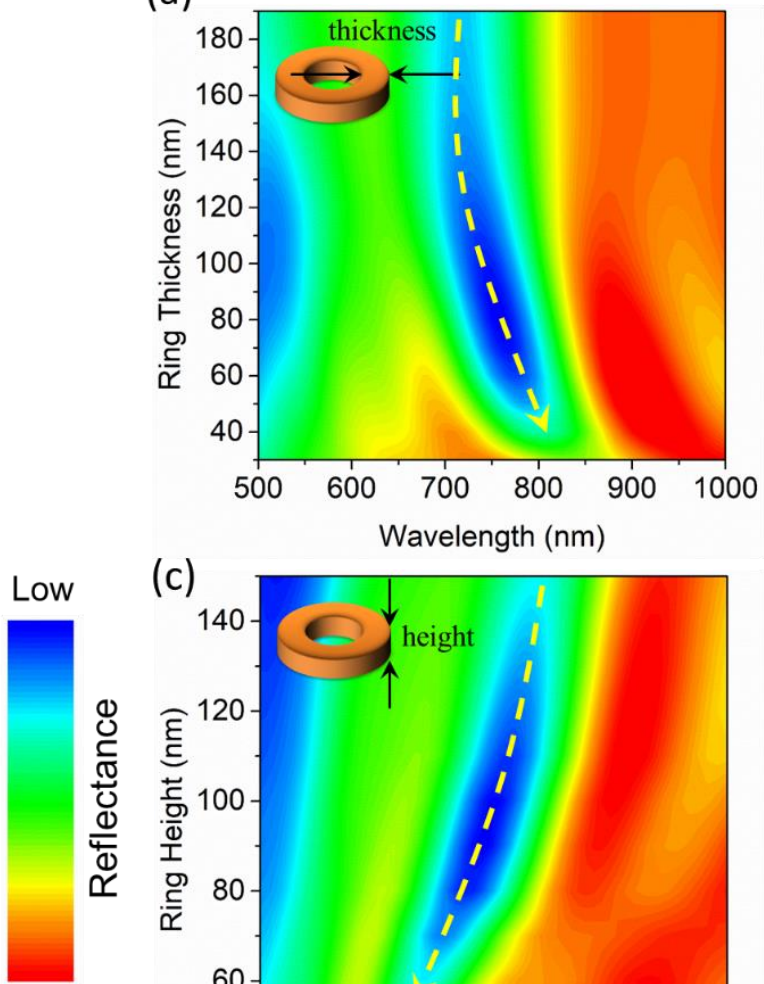

High

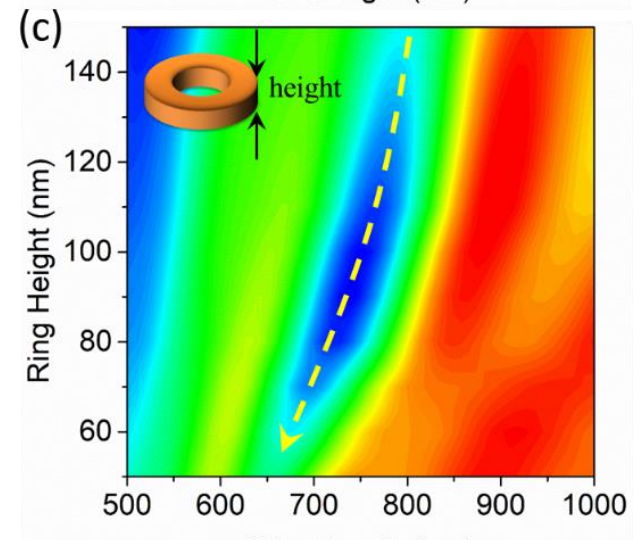

(e)

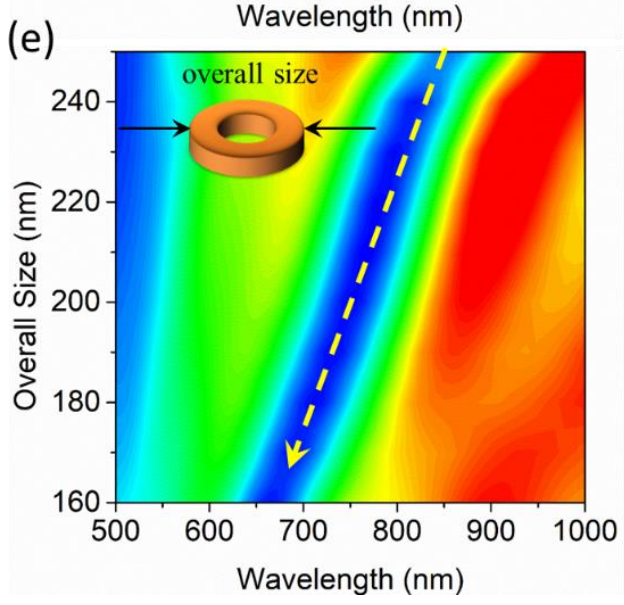

(b)
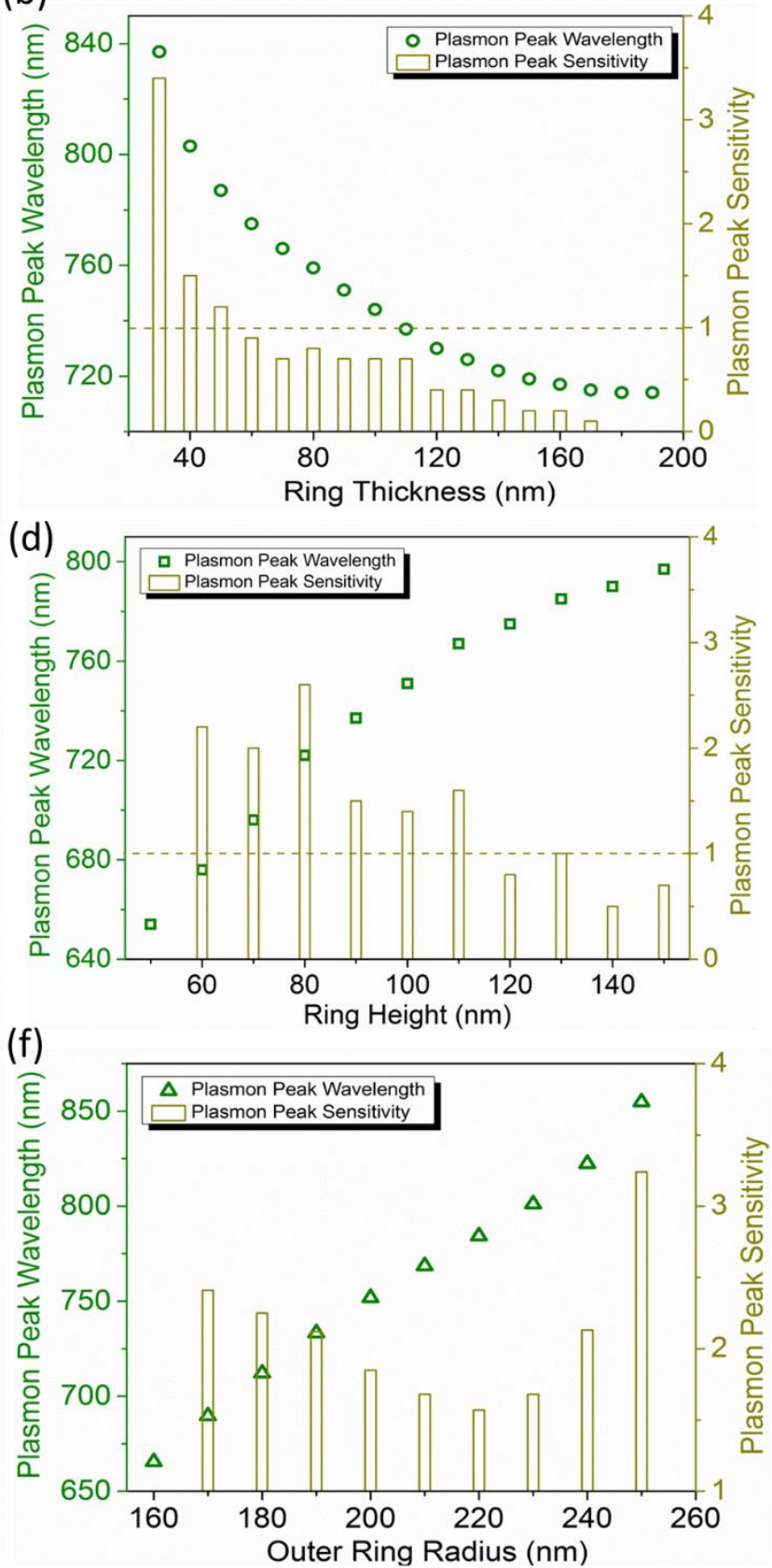

Figure 3.4. Optimization of optical properties of Au nanoring arrays. Dependence of the plasmon resonance peak on (a) the ring thickness, (c) the height, and (e) the overall thickness. The yellow dashed arrow shows the trend of the plasmon peak position evolution. Plot of the plasmon peak sensitivity with respect to each tuning parameter extracted from Figure $4(a)$, (b), and (c). The plasmon peak sensitivity is defined as the plasmon peak wavelength change for every $1 \mathrm{~nm}$ 
variation of each tuning parameter. The refractive index used for the Si substrate in FDTD simulations is 3.42 .

\section{Optimization of Au nanoring array patterns}

To achieve an optimal performance in surface-enhanced spectroscopies, the plasmonic band of the nanostructure needs to be overlapped with the spectral wavelength of the excitation laser source, and the local EM field enhancement needs to be maximized. The spectral overlap between the excitation light source and the SPR band ensures a resonance excitation; and the local EM field enhancement helps modulate various near-field optical processes. ${ }^{29-31}$ As identified above, the manifold parameters such as the ring thickness, height and overall ring size are found to affect the optical properties of the Au nanoring array patterns. Therefore, they all need to be studied and optimized.

In case of the Au nanoring arrays with a large gap where plasmonic coupling among neighboring rings can be ignored, the behavior of individual nanorings just needs to be considered and the periodicity of nanoring had no effect, as shown in Figure 3.4(a), 3.4(c) and 3.4(e). For any individual nanoring, among the parameters of the ring thickness, height and overall ring size, one was varied with all the other parameters fixed to study the plasmonic properties using FDTD simulation. For example, Figure 3.4(a) shows the effect of the ring thickness on the spectral wavelength (position) of plasmon peak in the meanwhile keeping the ring height, the overall ring size and the periodicity constant. A decrease in the ring thickness was found to red-shift the plasmon peak because of an increasingly stronger plasmonic coupling between charges at the outer and inner surfaces of the ring. A similar red-shift behavior of the plasmon peak was found for an increasing the ring height and the overall ring size, which was characteristic of the dipolar plasmon resonance of nanoparticles. It was found that the plasmon peak can be tuned 100 200 nm by merely varying one of the three tuning parameters above. The wide spectral tunability of $\mathrm{Au}$ nanoring arrays allows the resonance excitation of SPR by overlapping the SPR band with the wavelength of the excitation laser source.

Dependence of the plasmon peak sensitivity on the tuning parameters was also studied to reveal the parameter regions where the optical properties are most sensitively affected. The plasmon peak sensitivity is defined as the plasmon peak shift for every $1 \mathrm{~nm}$ change of each tuning 
parameter. The plasmon peak was found to be more sensitive at a smaller ring thickness, as shown in Figure 3.4(b). At a small ring thickness, the plasmonic coupling between charges at the outer and inner surfaces of the ring became weaker as an increase in the ring thickness. At a large ring thickness, there was no plasmonic coupling between charges at the outer and inner surfaces of the ring, which explained why varying the ring thickness barely shifted the plasmon at larger ring thicknesses. The plasmon peak was found to be largely equally sensitive to the ring height and overall ring size in a wide parameter region since the spectral dependence of the plasmon peak on both tuning parameters scale linearly, as shown in Figure 3.4(d) and 3.4(f).

For the Au nanoring arrays where plasmonic coupling among neighboring rings is strong, the plasmonic properties were studied in a pair of rings by varying the gap, as shown in Figure 3.5. The plasmonic coupling between two approaching rings was found to become increasingly intensified, as manifested by the split of the plasmon peak into two new peaks corresponding to a bonding SPR mode and an antibonding SPR mode as shown in Figure 3.5(a), which was also experimentally observed in Figure 3.5(b). When the gap of the Au nanoring array with a period of $500 \mathrm{~nm}$ changed from $100 \mathrm{~nm}$ to $2 \mathrm{~nm}$, the plasmon peak at $750 \mathrm{~nm}$ was split to two peaks (i.e., the antibonding SPR peak at $700 \mathrm{~nm}$ and the bonding SPR peak at $920 \mathrm{~nm}$ ), as shown in Figure 3.5(a). For the Au nanoring array with a periodicity of $600 \mathrm{~nm}$, reducing the gap from $90 \mathrm{~nm}$ to 12 $\mathrm{nm}$ made the $850 \mathrm{~nm}$ peak split to the antibonding SPR peak at $790 \mathrm{~nm}$ and the bonding SPR peak at $900 \mathrm{~nm}$ Figure 3.5(b). 
(a)

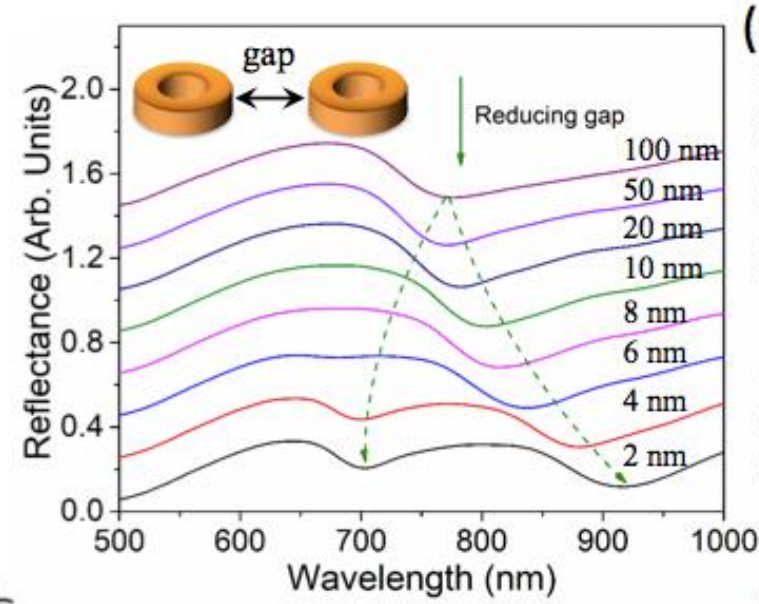

(c)

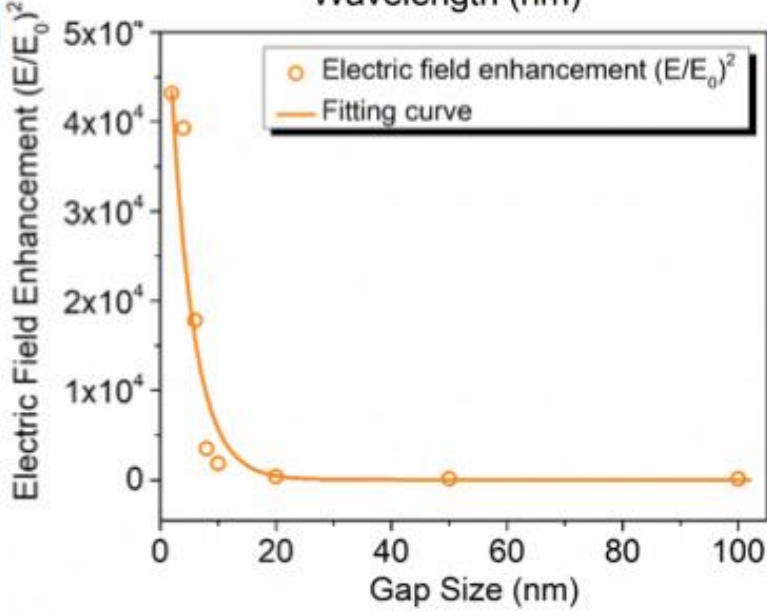

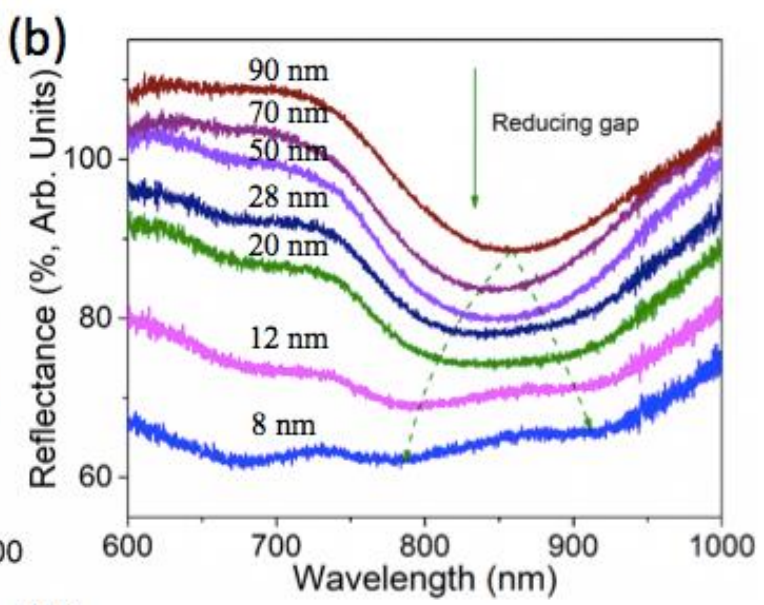

(d)

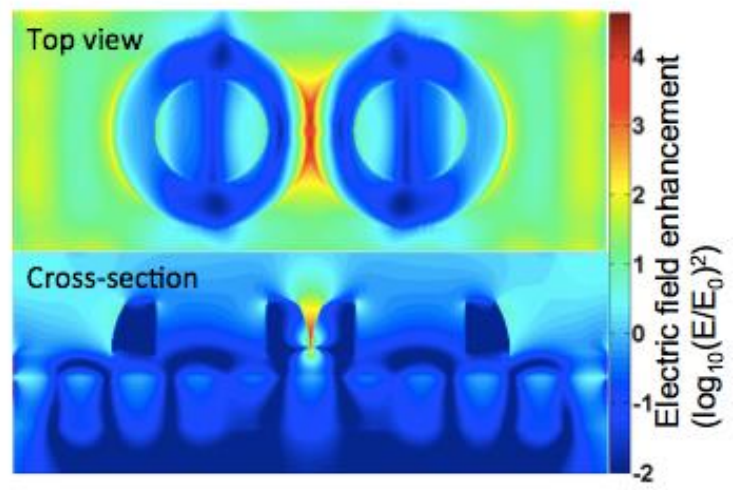

Figure 3.5. Plasmonic coupling between a pair of rings. (a) Simulated reflection spectra for the Au nanoring array with a periodicity of $500 \mathrm{~nm}$; (b) experimental reflection spectra for the Au nanoring array with a periodicity of $600 \mathrm{~nm}$. The olive dashed arrow shows the splitting of the plasmon peak into a bonding resonance mode (longer wavelength) and an anti-bonding resonance mode (shorter wavelength); (c) Gap-dependent EM field enhancement of a pair of Au rings; (d) the EM field distribution of a pair of rings with a gap of $2 \mathrm{~nm}$. The magnitude of the EM field enhancement in $(d)$ is presented as $\log _{10}\left(E / E_{0}\right)^{2}$.

In case of the $\mathrm{Au}$ nanoring arrays with a large gap where plasmonic coupling among neighboring rings can be ignored, the local EM field enhancement $\left(E^{2} / E_{0}\right)^{2}$ for an individual nanoring was only $\sim 5.0 \times 10^{2}$, as shown in Figure 3.3(b). In case of the Au nanoring arrays with a small gap where plasmonic coupling among neighboring rings occurred, the simulated local EM field enhancement $\left(\mathrm{E}_{\mathrm{E}} \mathrm{E}_{0}\right)^{2}$ increased significantly with a decrease in the gap, reaching $4.3 \times 10^{4}$ at a gap of $2 \mathrm{~nm}$ as shown in Figure 3.5(c). The corresponding local EM field distribution transited 
from being localized at the outer surface of the ring to the increasingly delocalized at the gap between neighboring rings, as shown in Figure 3.5(d). The local EM field enhancement can significantly mediate the performance of Au nanoring arrays in surface-enhanced spectroscopies through near-field interaction. However, maximizing the local EM field enhancement by coupling neighboring rings shifts the plasmon peak and thus could result in the breakdown of the resonance excitation.

\section{Detection of miRNA}

The Aunanostar@MBA@ $\mathrm{SiO}_{2}$ core shell structures are used to further improve the SERS enhancement from coupling from the tips of nano star with the gold nano ring array. To detect target miRNA, the $\mathrm{Au}$ nanostar@MBA@ $@ \mathrm{SiO}_{2}$ nanoparticles and the $\mathrm{Au}$ nanoring chips were modified with detection probe and capture probe respectively. When the miRNAs were absent in the aqueous solution, the two ssDNAs cannot hybridize with miRNA. Thus SERS probe with Raman reporter can be easily washed away from the substrate resulting in no signal. When there were miRNAs, two ssDNAs partially hybridize with miRNA resulting in a sandwich structure when the SERS probe is in close proximity to the substrate. This results in the Raman signal of MBA molecule confirming the miRNA presence.
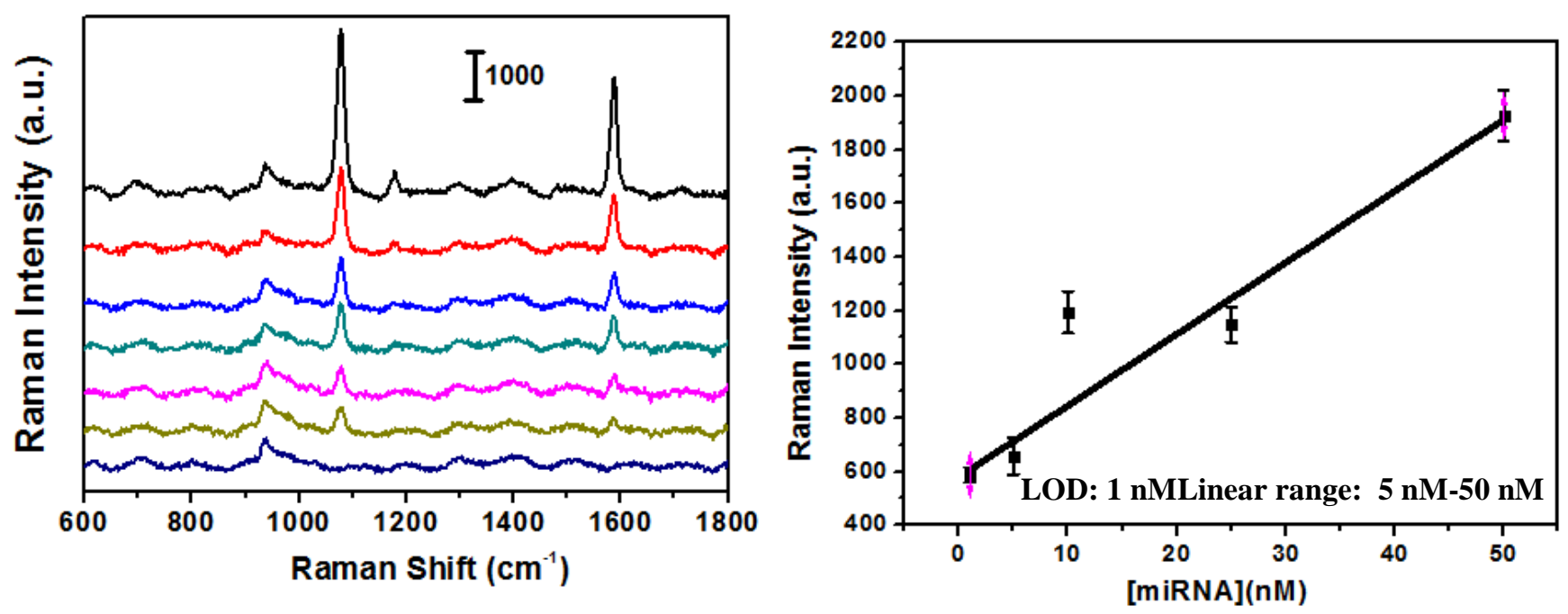

Figure 3. 6: (a) Raman spectrum of Aunanostar@ $M B A @ S_{2} O_{2}$ (b) Raman Intensity vs miRNA concentration to find limit of detection 
During the detection, firstly, $10 \mathrm{uL}$ of detection probe functionalized Au nanostar@ $\mathrm{MBA} @ \mathrm{SiO}_{2}$ nanoparticle solution was dropped on the capture probe labeled Au nanoring chip. Then $10 \mathrm{uL}$ of the sample solution containing a certain amount of miRNA was also dropped on the chips. After 20 min incubation, the Au nanoring chip was rinsed with a buffer solution, and then dried with a nitrogen flow. Subsequently, the Raman spectrum was acquired.

\section{Guideline for fabrication of optimized Au nanoring array patterns}

The analysis above reveals two approaches to optimize Au nanoring arrays for surfaceenhanced spectroscopy. First, the wide spectral tuning range allows the resonant excitation of SPR by overlapping the excitation wavelength with the SPR band. This is made possible by varying either the ring thickness, height, or the overall ring size. The three tuning curves obtained represented as yellow dashed arrows in Figure 3.4 provide a general guideline in choosing the optimal parameters for $\mathrm{Au}$ nanoring array fabrication. Second, the strong local EM field enhancement can be achieved on the closely packed Au nanoring arrays due to plasmonic coupling. It is noted that maximizing local EM field enhancement could break down the resonance excitation since the plasmonic coupling could shift the plasmon resonance peak, making no spectral overlap. Therefore, to achieve an overall optimized performance, a compromise could be made to balance the resonance excitation and the local EM field enhancement, depending on the weight of each contribution to surface-enhanced spectroscopy.

\subsection{CONCLUSIONS}

In summary, the large-area long-range ordered Au nanoring array patterns were fabricated using the modified NSL technique. The plasmon resonance peak position can be tuned in large spectral range by the manifold parameters such as the ring thickness, the ring height, and the overall ring size. For example, the plasmon resonance peak position can be controlled to be spectrally overlapped with the wavelength $(785 \mathrm{~nm})$ of the excitation laser, which is widely used in SERS sensors. The local EM field enhancement can be achieved nearby the outer and inner surface of individual nanorings and became stronger with a decrease in the gap between neighboring nanorings by forming the "hot spots". For maximizing the enhancement effect in SERS or SPCE, the contributions from the resonance excitation (spectral overlap) and the local 
EM field enhancement need to be balanced. The knowledge obtained can guide the design of optical and optoelectronic devices in various surface-enhanced spectroscopy. By carefully balancing contributions from excitation and local EM field enhancement, an overall optimized performance can be achieved. $1 \mathrm{nM}$ of mi-RNA is detected using gold nano ring array in coupled ring array pattern. The knowledge obtained in this research can guide the design of optical and optoelectronic devices in various surface-enhanced spectroscopies. 


\section{References}

[1] Willets, K. A.; Van Duyne, R. P. Localized surface plasmon resonance spectroscopy and sensing. Annu. Rev. Phys. Chem. 2007, 58, 267-297.

[2] Stiles, P. L.; Dieringer, J. A.; Shah, N. C.; Van Duyne, R. R. Surface-Enhanced Raman Spectroscopy. Annu. Rev. Anal. Chem. 2008, 1, 601-626.

[3] Le Ru, E. C.; Etchegoin, P. G. Single-Molecule Surface-Enhanced Raman Spectroscopy. Annu. Rev. Phys. Chem. 2012, 63, 65-87.

[4] Cao, S. H.; Cai, W. P.; Liu, Q.; Li, Y. Q. Surface Plasmon-Coupled Emission: What Can Directional Fluorescence Bring to the Analytical Sciences? Annu. Rev. Anal. Chem. 2012, 5, 317336.

[5] Gryczynski, I.; Malicka, J.; Gryczynski, Z.; Lakowicz, J. R. Surface plasmon-coupled emission with gold films. J. Phys. Chem. B 2004, 108, 12568-12574.

[6] Li, M.; Cushing, S. K.; Zhang, J. M.; Lankford, J.; Aguilar, Z. P.; Ma, D. L.; Wu, N. Q. Shapedependent surface-enhanced Raman scattering in gold-Ramanprobe-silica sandwiched nanoparticles for biocompatible applications. Nanotechnology 2012, 23, 115501.

[7] Zheng, P.; Li, M.; Jurevic, R.; Cushing, S. K.; Liu, Y. X.; Wu, N. Q. A gold nanohole array based surface-enhanced Raman scattering biosensor for detection of silver(I) and mercury(II) in human saliva. Nanoscale 2015, 7, 11005-11012.

[8] Zheng, P.; Cushing, S. K.; Suri, S.; Wu, N. Q. Tailoring plasmonic properties of gold nanohole arrays for surface-enhanced Raman scattering. Phys. Chem. Chem. Phys. 2015, 17, 21211-21219. [9] Sherry, L. J.; Jin, R. C.; Mirkin, C. A.; Schatz, G. C.; Van Duyne, R. P. Localized surface plasmon resonance spectroscopy of single silver triangular nanoprisms. Nano Lett. 2006, 6, 20602065.

[10] Haes, A. J.; Van Duyne, R. P. A nanoscale optical blosensor: Sensitivity and selectivity of an approach based on the localized surface plasmon resonance spectroscopy of triangular silver nanoparticles. J. Am. Chem. Soc. 2002, 124, 10596-10604.

[11] Khoury, C. G.; Norton, S. J.; Vo-Dinh, T. Plasmonics of 3-D Nanoshell Dimers Using Multipole Expansion and Finite Element Method. Acs Nano 2009, 3, 2776-2788.

[12] Petoukhoff, C. E.; O'Carroll, D. M. Absorption-induced scattering and surface plasmon outcoupling from absorber-coated plasmonic metasurfaces. Nat. Commun. 2015, 6, 7899. 
[13] Toma, M.; Cho, K.; Wood, J. B.; Corn, R. M. Gold Nanoring Arrays for Near Infrared Plasmonic Biosensing. Plasmonics 2014, 9, 765-772.

[14] Scheeler, S. P.; Lehr, D.; Kley, E. B.; Pacholski, C. Top-Up Fabrication of Gold Nanorings. Chem. Asian J. 2014, 9, 2072-2076.

[15] Halpern, A. R.; Corn, R. M. Lithographically Patterned Electrodeposition of Gold, Silver, and Nickel Nanoring Arrays with Widely Tunable Near-Infrared Plasmonic Resonances. Acs Nano 2013, 7, 1755-1762.

[16] Tsai, C. Y.; Lu, S. P.; Lin, J. W.; Lee, P. T. High sensitivity plasmonic index sensor using slablike gold nanoring arrays. Appl. Phys. Lett. 2011, 98, 153108.

[17] Teo, S. L.; Lin, V. K.; Marty, R.; Large, N.; Alarcon-Llado, E.; Arbouet, A.; Girard, C.; Aizpurua, J.; Tripathy, S.; Mlayah, A. Gold nanoring trimers: a versatile structure for infrared sensing. Opt. Express 2010, 18, 22271-22282.

[18] Ye, J.; Shioi, M.; Lodewijks, K.; Lagae, L.; Kawamura, T.; Van Dorpe, P. Tuning plasmonic interaction between gold nanorings and a gold film for surface enhanced Raman scattering. Appl. Phys. Lett. 2010, 97, 163106.

[19] Aizpurua, J.; Hanarp, P.; Sutherland, D. S.; Kall, M.; Bryant, G. W.; de Abajo, F. J. G. Optical properties of gold nanorings. Phys. Rev. Lett. 2003, 90, 057401.

[20] Alali, F.; Karampelas, I. H.; Kim, Y. H.; Furlani, E. P. Photonic and Thermofluidic Analysis of Colloidal Plasmonic Nanorings and Nanotori for Pulsed-Laser Photothermal Applications. The Journal of Physical Chemistry C 2013, 117, 20178-20185.

[21] Hu, Y.; Chou, T.; Wang, H.; Du, H. Monodisperse Colloidal Gold Nanorings: Synthesis and Utility for Surface-Enhanced Raman Scattering. The Journal of Physical Chemistry C 2014, 118, 16011-16018.

[22] Prieto, M.; Arenal, R.; Henrard, L.; Gomez, L.; Sebastian, V.; Arruebo, M. Morphological Tunability of the Plasmonic Response: From Hollow Gold Nanoparticles to Gold Nanorings. The Journal of Physical Chemistry C 2014, 118, 28804-28811.

[23] Jiang, H.; Sabarinathan, J. Effects of Coherent Interactions on the Sensing Characteristics of Near-Infrared Gold Nanorings. The Journal of Physical Chemistry C 2010, 114, 15243-15250.

[24] Barik, A.; Otto, L. M.; Yoo, D.; Jose, J.; Johnson, T. W.; Oh, S.-H. DielectrophoresisEnhanced Plasmonic Sensing with Gold Nanohole Arrays. Nano Lett 2014, 14, 2006-2012. 
[25] Liu, M.; Song, Y.; Zhang, Y.; Wang, X.; Jin, C. Mode Evolution and Transmission Suppression in a Perforated Ultrathin Metallic Film with a Triangular Array of Holes. Plasmonics 2012, 7, 397-410.

[26] Cho, K.; Loget, G.; Corn, R. M. Lithographically Patterned Nanoscale Electrodeposition of Plasmonic, Bimetallic, Semiconductor, Magnetic, and Polymer Nanoring Arrays. The Journal of Physical Chemistry C 2014, 118, 28993-29000.

[27] Palik, E. D., Handbook of optical constants of solids. Academic Press: Orlando, 1985.

[28] Prodan, E.; Radloff, C.; Halas, N. J.; Nordlander, P. A hybridization model for the plasmon response of complex nanostructures. Science 2003, 302, 419-422.

[29] Urena, E. B.; Kreuzer, M. P.; Itzhakov, S.; Rigneault, H.; Quidant, R.; Oron, D.; Wenger, J. Excitation Enhancement of a Quantum Dot Coupled to a Plasmonic Antenna. Adv. Mater. 2012, 24, 314-320.

[30] Schlucker, S. Surface-Enhanced Raman Spectroscopy: Concepts and Chemical Applications. Angew. Chem. Int. Ed. Engl. 2014, 53, 4756-4795.

[31] Gu, Y.; Wang, L. J.; Ren, P.; Zhang, J. X.; Zhang, T. C.; Martin, O. J. F.; Gong, Q. H. SurfacePlasmon-Induced Modification on the Spontaneous Emission Spectrum via SubwavelengthConfined Anisotropic Purcell Factor. Nano Lett. 2012, 12, 2488-2493. 


\section{CHAPTER 4: LIGHT MANAGEMENT AND PLEXITON CREATION BY PLASMONIC GAP MODE}

Light management is given utmost importance to improve performance of optoelectronic devices. The structure design has a large impact on the efficiency of the device. This chapter presents a facile microfabrication-compatible approach to fabricate the large area of plasmonic nano-pyramid array-based antennas and demonstrates effective light management by tailoring the architecture. Firstly, a long-range ordered gold nano-pyramid array is fabricated, which exhibits strong light scattering. The maximum electric field enhancement $\left(|\mathbf{E}| /\left|\mathbf{E}_{0}\right|\right)$ of 271 is achieved at the corner but decays rapidly away from the pyramid bottom. After the gold nanopyramid array is coupled to a gold film, strong light scattering is converted into strong light absorption due to the excitation of a spectrally tunable plasmonic gap mode, where an intense electric field enhancement of 233 and a strong magnetic field enhancement $\left(|\mathbf{H}| /\left|\mathbf{H}_{0}\right|\right)$ of 25 are simultaneously excited for a $10 \mathrm{~nm}$ silica gap. The electric field decays much slower away from the pyramid bottom while the magnetic field keeps almost constant. The nanostructure is predicted to find extensive applications in optoelectronic devices. For example, if quantum emitters such as J-aggregates are embedded in the gap, strong coupling between J-aggregates and the plasmonic gap mode could take place, which was confirmed experimentally and by finite-difference time-domain simulations. This can overcome the problems of high energy loss and weak nonlinearity, which are typically associated with surface plasmon polariton (SPP) supported on plasmonic metallic nanostructures. 


\subsection{INTRODUCTION}

While fundamental to photosynthesis, photoenergy conversion, optical biosensing, superresolution microscopy, et al, ${ }^{1-4}$ light-matter interactions are difficult to realize because of the fundamental mismatch between the diffraction-limited spatial confinement of visible light and the size of quantum emitters (QEs). ${ }^{5}$ The diffraction limit, however, can be broken by surface plasmon polaritons (SPPs), which are light-coupled propagating EM waves of coherent electron oscillations on metallic surfaces. ${ }^{6}$ SPPs comes with two prominent features. ${ }^{7-8}$ First, it belongs to bosons. Therefore, it has a strong transportability of the EM energy. Second, it has the most harmonic behavior known in nature. Unfortunately, the plasmonic cavity which supports SPPs falls short of essential functionalities in modern optoelectronic devices owing to a very weak nonlinear response and a high metal loss. ${ }^{9-10}$ Coincidently, QEs are highly nonlinear in nature despite a low transportability. ${ }^{11}$ They are extensively exploited to realize optical gain in lasers and other optoelectronic devices. ${ }^{12-16}$ The integrated SPPs-QEs system can thus well balance and compensate the nonlinearity and transportability of the two counterparts except that a strong damping from the metal loss persists.

One promising approach to overcome the metal loss is to make conditions for the interaction of the SPPs-QEs system to take place in the strong-coupling regime. ${ }^{17-25}$ The coupling strength $g$ is characterized by Rabi frequency $\Omega_{R}\left(\Omega_{R}=2 g\right)$, which quantifies the rate of energy exchange between the plasmonic cavity and QEs. ${ }^{26}$ In the strong-coupling regime, the coupling strength is so strong that it overwhelms the damping rate $\kappa$ of the plasmonic cavity and the spontaneous decay rate $\tau_{0}$ of QEs, i.e. $g>\kappa, \tau_{0}$. The extraordinary coupling strength facilitates an extremely rapid, coherent, and reversible energy exchange between the plasmonic cavity and QEs, leading to two newly formed hybridized states called upper polariton and lower polariton which are half-light, half-matter, and are split by Rabi frequency. ${ }^{5,10,26}$ Given the dependence of the hybridized states on the coupling strength, the strongly-coupled SPPs-QEs system has strong implications in stateof-the-art optoelectronic devices such as ultrafast switching, all-optical control, et al. ${ }^{27-29}$ In comparison, in the weak-coupling regime where the damping rate of the plasmonic cavity outcompetes the spontaneous decay rate of QEs and the coupling strength, i.e. $\kappa>\tau_{0}, g$, the interaction is incoherent and irreversible. ${ }^{5,10,26}$ The energy extracted from QEs is unidirectionally 
transferred to the cavity with the spontaneous decay rate enhanced by the cavity-dependent Purcell factor.

Realizing strong coupling demands an intense interaction between the transition dipole $\mu$ of QEs and the plasmonic cavity: $g \propto \mu \sqrt{N / V}$, where $V$ is the effective mode volume of the plasmonic cavity in which the EM energy is confined and $N$ is the number of QEs in $V .{ }^{5,10,26}$ The diffractionunlimited nature of SPPs allows light to be confined in a significantly smaller plasmonic cavity than the photonic counterpart until the emergence of charge transfer plasmons due to quantum tunneling or nonlocal effects at a gap below $1 \mathrm{~nm} \cdot{ }^{30-33}$ Such extraordinarily small plasmonic cavities have been demonstrated on nanoparticle-on-mirror geometry, bowtie antennas, patch antenna, et al. ${ }^{17,34-35}$ Minimizing the effective mode volume, however, is also at the expense of reducing the number of QEs participating light-matter interactions, which compromises the coupling strength. Moreover, it is also a technique challenge to fabricate ultra-small plasmonic cavity. Therefore, from a practical point of view, modern optoelectronic devices working in the strong-coupling regime require a precise fabrication for better performance.

In this work, the nanostructure of film-coupled gold nanopyramid array is proposed, in which the hexagonally-patterned gold nanopyramid arrays sit on an underlying gold film separated by a thickness-tunable spacer layer. There are many advantages with this nanostructure. First, it supports plasmonic gap modes, which are tunable both spectrally and in terms of the effective mode volume. Second, QEs at the spacer layer exactly fall into the effective mode volume, which can ensure the largest possibly achievable coupling strength. Third, it can be fabricated on a large scale by the well-established and cost-effective technique of nanosphere lithography in the cleanroom. ${ }^{36}$ The spacer layer can be easily replaced with any other QEs of interest to realize different functionalities. Fourth, the long-range order of the structure ensures the robustness and reproducibility of fabrication. In addition, the two-dimensional nanostructure makes it practically easy to be integrated into optoelectronic devices. Therefore, in what follows, a combination of cleanroom fabrications and numeric simulations based on finite-difference time-domain (FDTD) method is implemented to study the optical properties of the film-coupled gold nanopyramid array. The capability of the nanostructure to manipulate light-matter interactions is also studied numerically by looking at the coupling between the plasmonic gap mode and QEs. 


\section{Fabrication of Gold Pyramid Array On Gold Film}

Figure 4.1 shows the procedure for fabrication of the Au nanopyramid array pattern directly on a glass substrate, and the Au nanopyramid array-silica-Au film structure, respectively. The Au nanopyramids were $179 \mathrm{~nm}$ in base edge length and $200 \mathrm{~nm}$ high. The silica space layer was controlled to be from $35 \mathrm{~nm}$ to $130 \mathrm{~nm}$. The overall lateral size of as-fabricated chips was typically comparable to that of a coin, as shown in the inset of Figure 4.2(a). This confirmed the strong capability of nanosphere lithography for long-range ordered nano-array nanofabrication. In contrast, it would take several days for e-beam lithography to produce such a large area of nanostructured patterns.

The proposed nanostructure of film-coupled Au nanopyramid array was fabricated by nanosphere lithography, as shown in Figure 4.1. Specifically, a thin layer with varying thicknesses of silica was first coated on a commercially available Au-coated glass slide by spin coating a layer of silica precursor (XR-1541) at varying speeds and then conducting heat treatment at $400{ }^{\circ} \mathrm{C}$ for 1 hour. Subsequently, a monolayer of polystyrene beads with a diameter of $600 \mathrm{~nm}$ was patterned on the silica surface by dip-coating. After natural drying in air, a layer of $5 \mathrm{~nm}$ Ti film was deposited, which was followed by depositing a layer of $200 \mathrm{~nm}$ Au film. After polystyrene beads were removed by ultrasonication, the film-coupled Au nanopyramid array was obtained. The entire fabrication process, which could be scaled up for mass production, was done in just one day in the cleanroom. Following a similar procedure, Au nanopyramid array was fabricated. 
(a) Gold-coated glass slides cleaning

(b) Silica film (varying thickness) coating

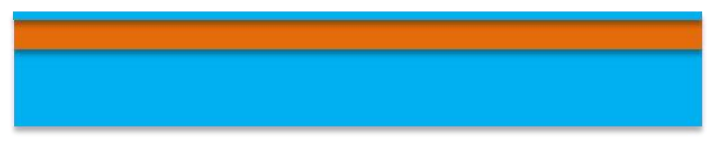

(c) Polystyrene beads (600 $\mathrm{nm}$ in diameter) patterning

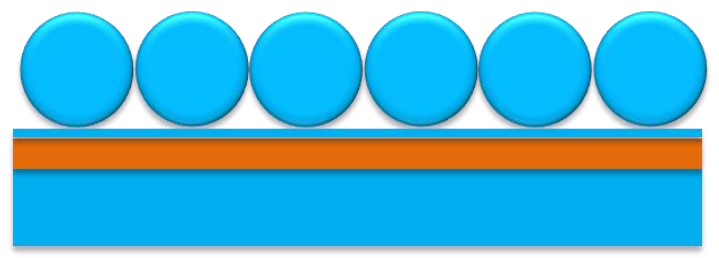

(d) Titanium film $(5 \mathrm{~nm})$ deposition

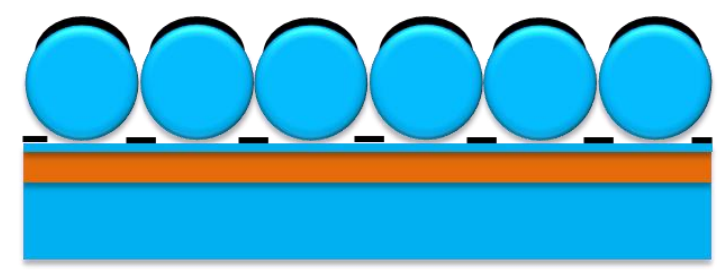

(e) Gold film (200 nm) deposition

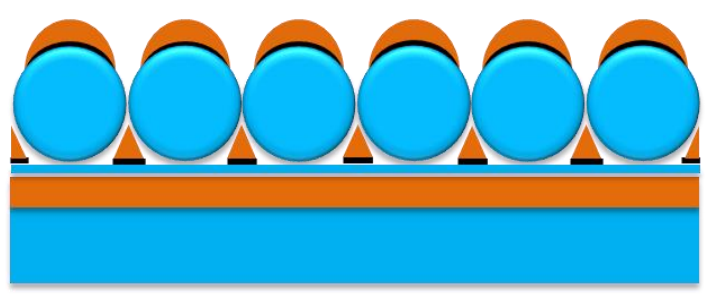

(f) Polystyrene beads removal

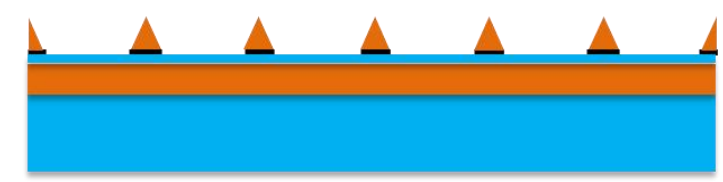

(g) Schematic film-coupled gold nanopyramid array

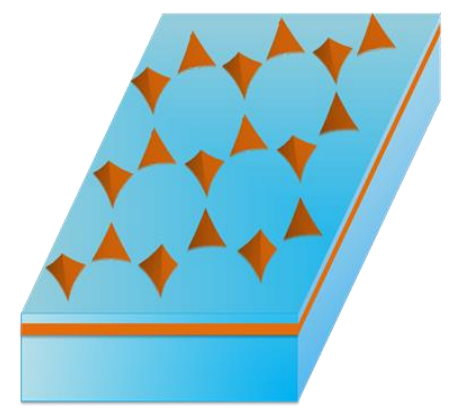

Figure 4. 1:Protocol of nanosphere lithography fabrication for film-coupled gold nanopyramid array. Polystyrene beads with a diameter of $600 \mathrm{~nm}$ were used. $200 \mathrm{~nm}$ of gold was deposited

\subsection{METHODS}

Chemicals and materials. Gold-coated glass slides were purchased from Fisher Scientific Inc. Polystyrene microspheres with a diameter of $600 \mathrm{~nm}$ were purchased from Thermo Scientific (Waltham, MA). XR1541 e-beam resist was purchased from Dow corning. Acetone, methanol, and sulphuric acid $\left(\mathrm{H}_{2} \mathrm{SO}_{4}\right)$ and hydrogen peroxide were purchased from Alfa Aesar (Haverhill, MA). Deionized (D.I.) water was produced by the Milli-Q Integral 3/5/10/15 system (18.2 M $\Omega$ cm, Millipore Corp., U.S.A.).

Nanosphere lithography fabrication: Figure 4.1 reveals the procedure for fabrication of the Au nanopyramid array-silica-Au film structure. Specifically, a thin layer with varying thicknesses of 
silica was first coated on a commercially available Au-coated glass slide by spin coating a layer of silica precursor (XR-1541) at varying speeds and then conducting heat treatment at $400{ }^{\circ} \mathrm{C}$ for 1 hour. Subsequently, a monolayer of polystyrene beads with a diameter of $600 \mathrm{~nm}$ was patterned on the silica surface by dip-coating. After natural drying in air, a layer of $5 \mathrm{~nm}$ Ti film was deposited, followed by depositing a $200 \mathrm{~nm}$ thick Au film. After polystyrene beads were removed by ultrasonication, the film-coupled Au nanopyramid array was obtained. The entire fabrication process, which could be scaled up for mass production, was done in just one day in the cleanroom.

Fabrication of Au film-J-aggregates-Au nanopyramid structure. $5 \mathrm{mg} / \mathrm{ml}$ of J-aggregates in water were mixed with $25 \mathrm{mg} / \mathrm{ml}$ of Poly Vinyl Alcohol (PVA) in water and let it stir for 3 hours. The mixture was spin coated on cleaned $1 \mathrm{~cm}$ x $1 \mathrm{~cm}$ gold coated glass chips at $4000 \mathrm{rpm}$ for $60 \mathrm{~s}$ to form a J-aggregate polymer thin film. A $5 \mathrm{~nm}$ thin silica layer was deposited on the top of Jaggregate polymer thin film for two reasons. Silica layer acts as a protective layer to prevent Jaggregates from damaging while depositing gold pyramids and it also helps in enabling a hydrophilic surface for dip coating where J-aggregate polymer thin film is hydrophobic in nature. Later 500nm Polystyrene beads monolayer was dip coated on the surface of silica. After drying in air, $5 \mathrm{~nm}$ Ti and 200nm Au films were deposited successively on the monolayer. Finally, the chips were ultra-sonicated in methanol to remove polystyrene beads and leaving Au nanopyramid array on the top layer.

Instrumentation. E-beam evaporator (Kurt J. Lesker) was used to deposit gold. Spin coater (Laurel Technologies 600 spinner) was used to coat the silica precursor to make the silica films with different thickness. A Scanning Electron Microscope (JEOL-JSM-7600F) was used to characterize fabricated film-coupled gold nanopyramid array. Reflection spectra were acquired by an Ocean Optics USB 4000 spectrometer and DT-MINI-2-GS.

Finite-difference time-domain (FDTD) simulations. FDTD simulations were implemented throughout the paper to study the film-coupled gold nanopyramid array using commercially available software Lumerical FDTD Solutions. A mesh size of $2 \mathrm{~nm}$ was imposed in the simulation region. The permittivity of gold was from Johnson \& Christy. ${ }^{55}$ The refractive index of silica was set as 1.52 . A continuous plane wave from $600 \mathrm{~nm}$ to $1000 \mathrm{~nm}$ was used as the input light source. Back reflection spectra, electromagnetic field enhancement, and surface polarization charges were 
calculated. Perfectly Matched Layer (PML) boundary conditions were imposed on all these simulations.

\subsection{RESULTS and DISCUSSIONS}

(a)

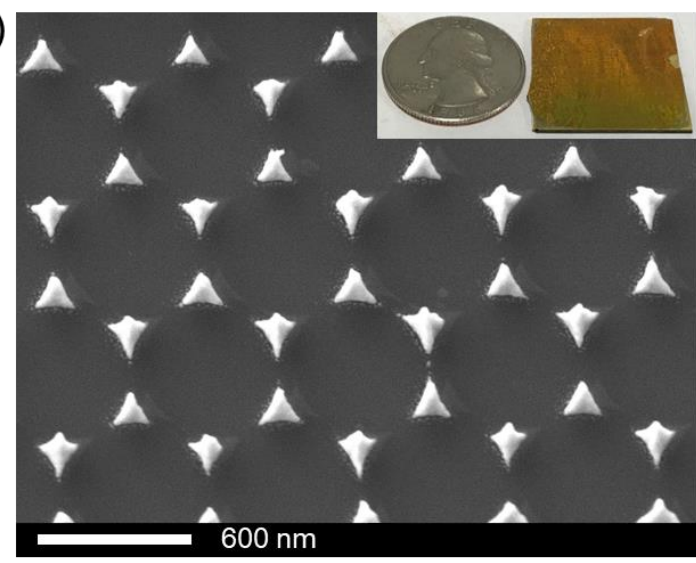

(c)

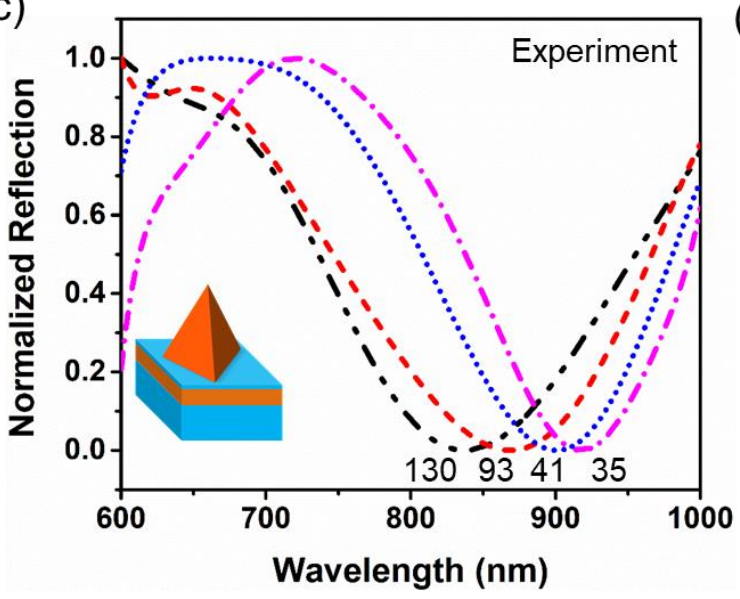

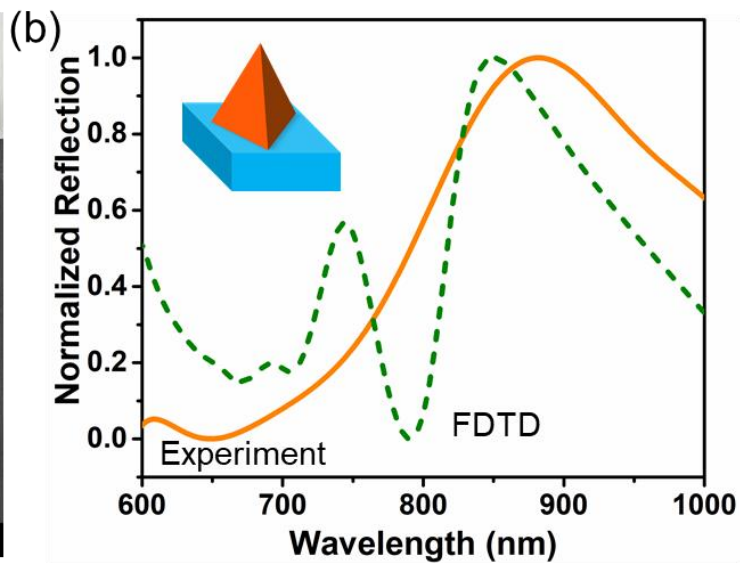

(d)

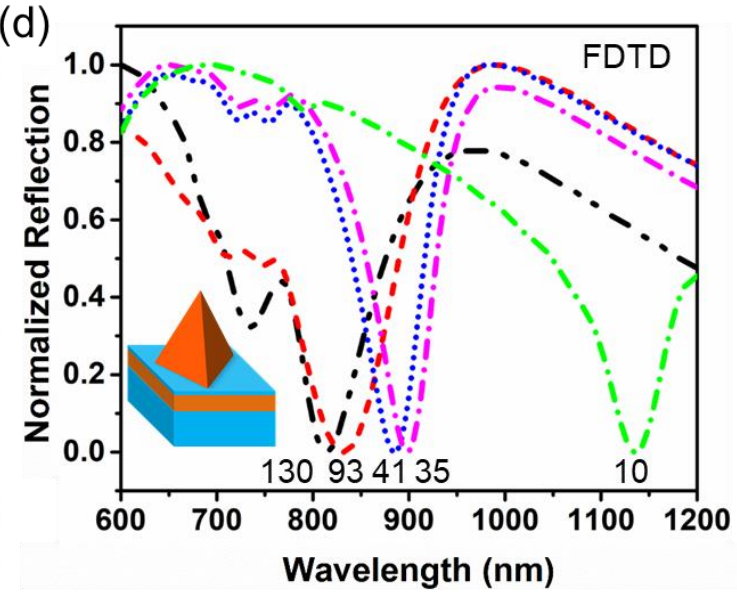

Figure 4. 2: (a) SEM image of fabricated film-coupled Au nanopyramid array (b) Experimental and FDTD-simulated reflection spectra for Au nanopyramid array (c) Experimental and (d) FDTD-simulated reflection spectra with various silica thicknesses

The fabricated Au nanopyramid array on the glass substrate displayed an intense reflection peak centered at $865 \mathrm{~nm}$ corresponding to the plasmonic corner mode, the orange solid curve as shown in Figure 4.2(b), which was consistent with the reflection peak at $850 \mathrm{~nm}$ calculated by FDTD, as shown with the olive dashed curve. The minor reflection peak at around $750 \mathrm{~nm}$ in the calculated 
reflection spectrum was ascribed to the edge mode, which was not clearly seen in the experimental data due to the rounded edge. The reflection peak contributed to strong light scattering of individual nanopyramids under excitation of the localized surface-plasmon resonance (LSPR) mode at the corner while light absorption by individual nanopyramids were small. It was not surprising because the size/volume of nanopyramids were quite large. For LSPR-associated total extinction spectra of nanoparticles, both light absorption and scattering contribute to the total extinction. ${ }^{6}$ When the particle size is relatively small (typically less than $15 \mathrm{~nm}$ ), light absorption is dominant. When the particle size increases, light scattering becomes dominate. In addition, the major reflection peak corresponding to the plasmonic corner mode red shifts with a decrease in the pyramid height. The FDTD simulation results show that the localized electric field enhancement took place primarily on the four corners, where surface polarization charges accumulate at the corners. As a result, the plasmonic corner mode exhibits intense electric field enhancement. The calculated maximum electric field enhancement $\left(|\mathbf{E}| /\left|\mathbf{E}_{\mathbf{0}}\right|\right)$ at the corners was estimated to be 271 .

\section{Transition from strong reflection to strong absorption}

Although the top-view SEM images looked the same for Au nanopyramid arrays with and without an underlying Au film, their optical properties differed significantly, as shown in Figure 4.2 (b), (c), and (d). For an ordinary Au nanopyramid array on glass, it displayed an intense reflection peak corresponding to the plasmonic corner mode, which was consistent with FDTD-simulated reflection spectrum, as shown in Figure 4.2(b). However, the intense reflection peak disappeared when the Au nanopyramid array was coupled with an underlying Au film separated by a silica spacer layer. Instead, the film-coupled Au nanopyramid array displayed a strong absorption, which

was featured as an intense reflection dip, as shown in Figure 4.2(c). By reducing the thickness of the silica spacer layer, the reflection dip underwent a red shift. This was also confirmed by FDTDsimulations, as shown in Figure 4.2(d). 


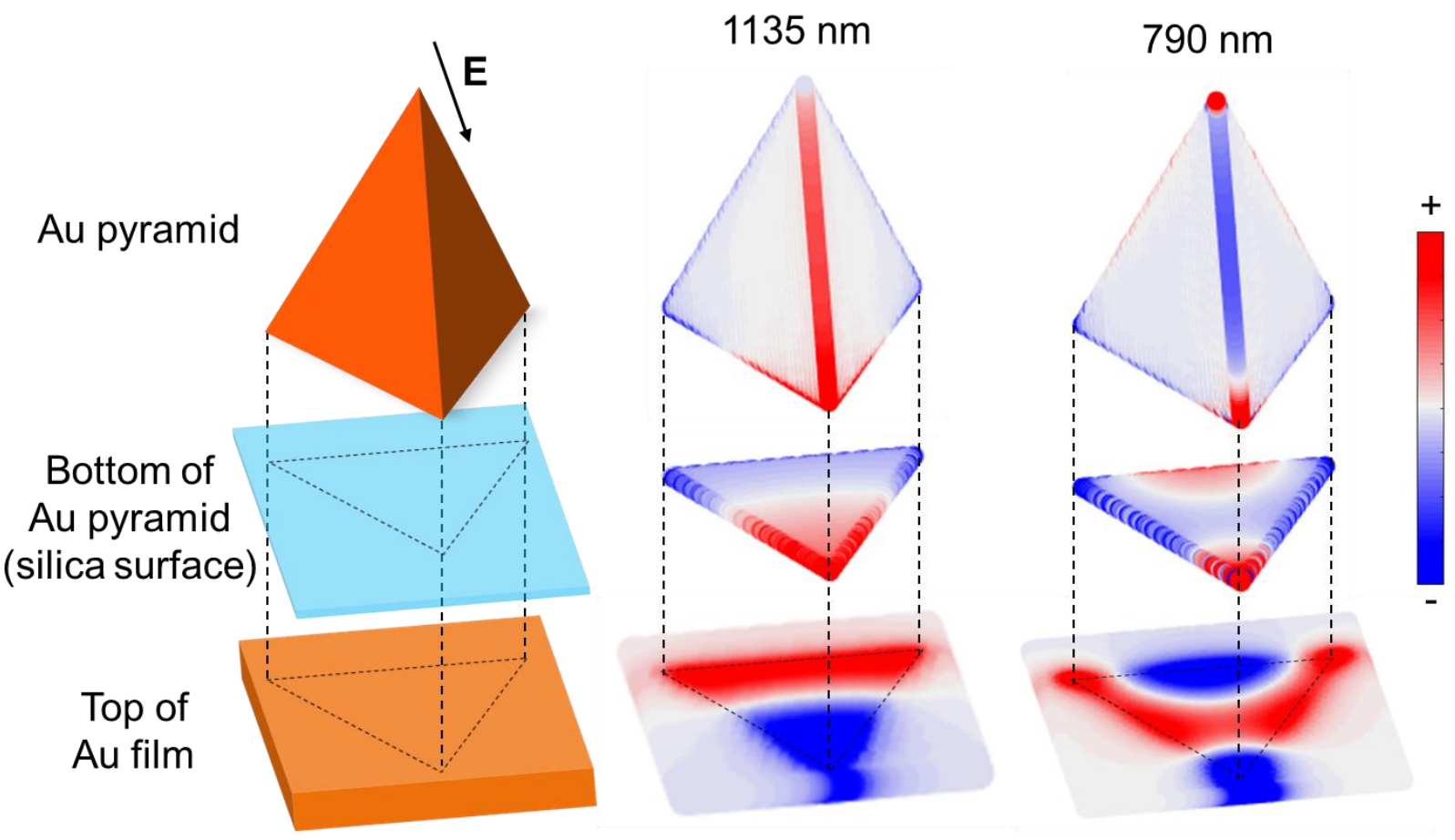

Figure 4. 3: FDTD-simulated surface polarization charge distributions at the reflection dip wavelengths of $1135 \mathrm{~nm}$ and $790 \mathrm{~nm}$ for a silica thickness of $10 \mathrm{~nm}$.

\section{Plasmonic gap mode of film-coupled gold nanopyramid array}

The observed intense reflection dip was ascribed to the formation of plasmonic gap modes at the spacer layer between the Au nanopyramid array and the underlying $\mathrm{Au}$ film. This was confirmed by calculations of surface polarization charge distributions in Figure 4.3 for a filmcoupled Au nanopyramid array with a spacer layer of $10 \mathrm{~nm}$ silica, which exhibited two reflection dips in $1135 \mathrm{~nm}$ and $790 \mathrm{~nm}$, as shown in the dash dotted curve in Figure 4.2(d). The primary plasmonic gap mode at $1135 \mathrm{~nm}$ was featured as a stronger reflection dip than the other, since the positive and negative surface polarization charges were well separated from each other, as shown in the middle column of Figure 4.3. The higher-order plasmonic gap mode at $790 \mathrm{~nm}$ displayed a less intense reflection dip because its net dipole momentum was partially cancelled, as shown in the right column of Figure 4.3. By varying the thickness of the spacer layer, the plasmonic gap modes could be spectrally tuned. In this paper, the study would mainly focus on the primary plasmonic gap mode, which will be just called plasmonic gap mode in all what follows. 


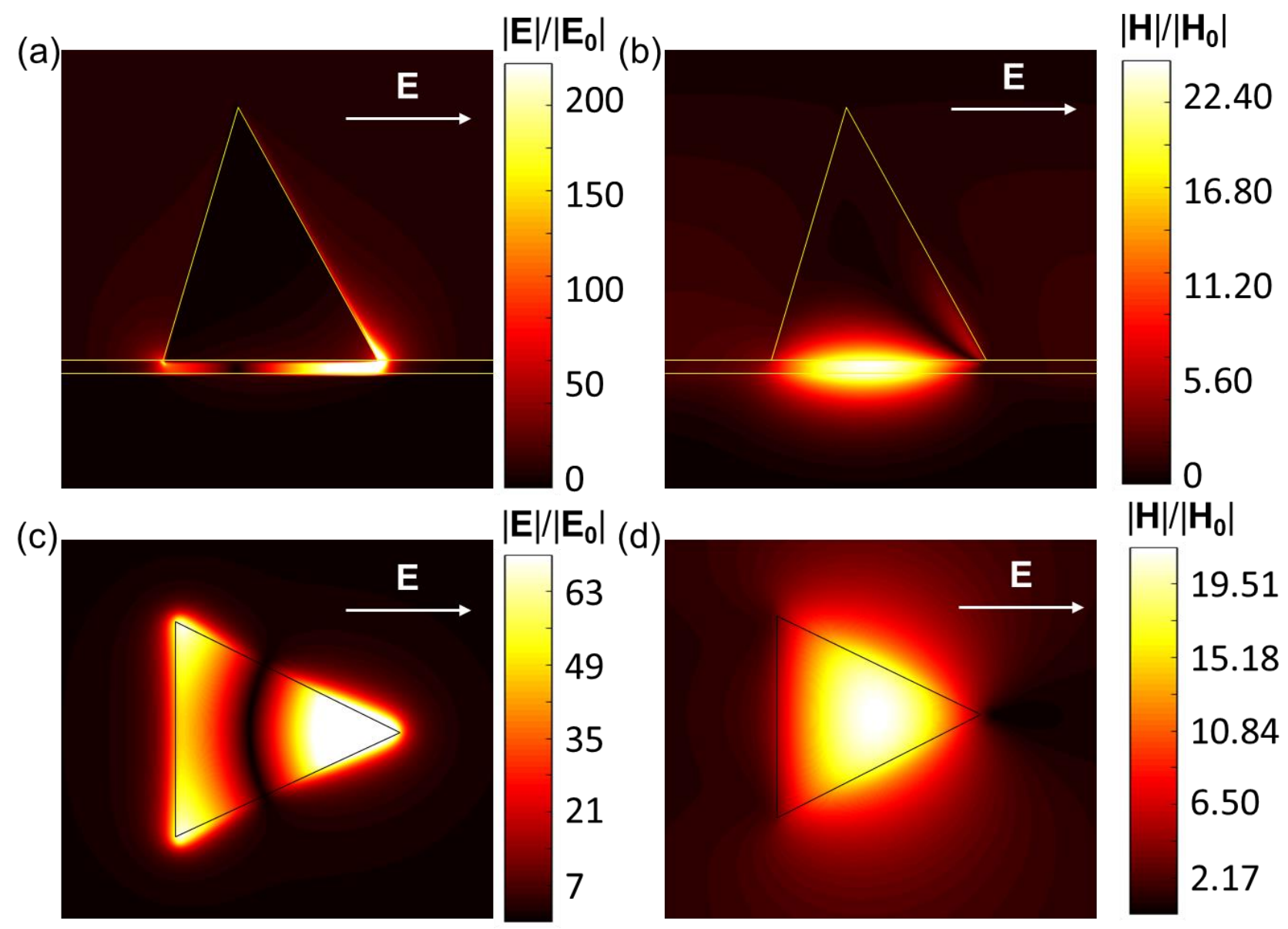

Figure 4. 4:(a) Electric and (b) magnetic field at the gap from a vertical cross-section view; (c) and $(d)$ Electric and magnetic fields at the center of the gap from a horizontal cross-section view

\section{Electromagnetic fields of plasmonic gap mode}

A prominent feature of the plasmonic gap mode was that charges with opposite signs were induced on opposite sides of the gap. These charges resulted in an intense electric field localized at the gap, as shown in Figure 4.4 (a) and (c). At the same time, the fictitious current loop along the periphery of the gap generated a strong magnetic field at the center, as shown in Figure 4.4 (b) and (d). Consequently, the localized EM energy oscillated back and forth between the electric and magnetic counterparts, and it only got damped either because of metal loss or by coupling to free space. The gap which confines the spatially oscillating EM energy thus defines a spectrally tunable plasmonic cavity with an effective mode volume approximately the actual volume of the gap region. This feature provides an excellent configuration to enable the maximum interaction between SPPs and the spacer layer at the gap. 
(a)
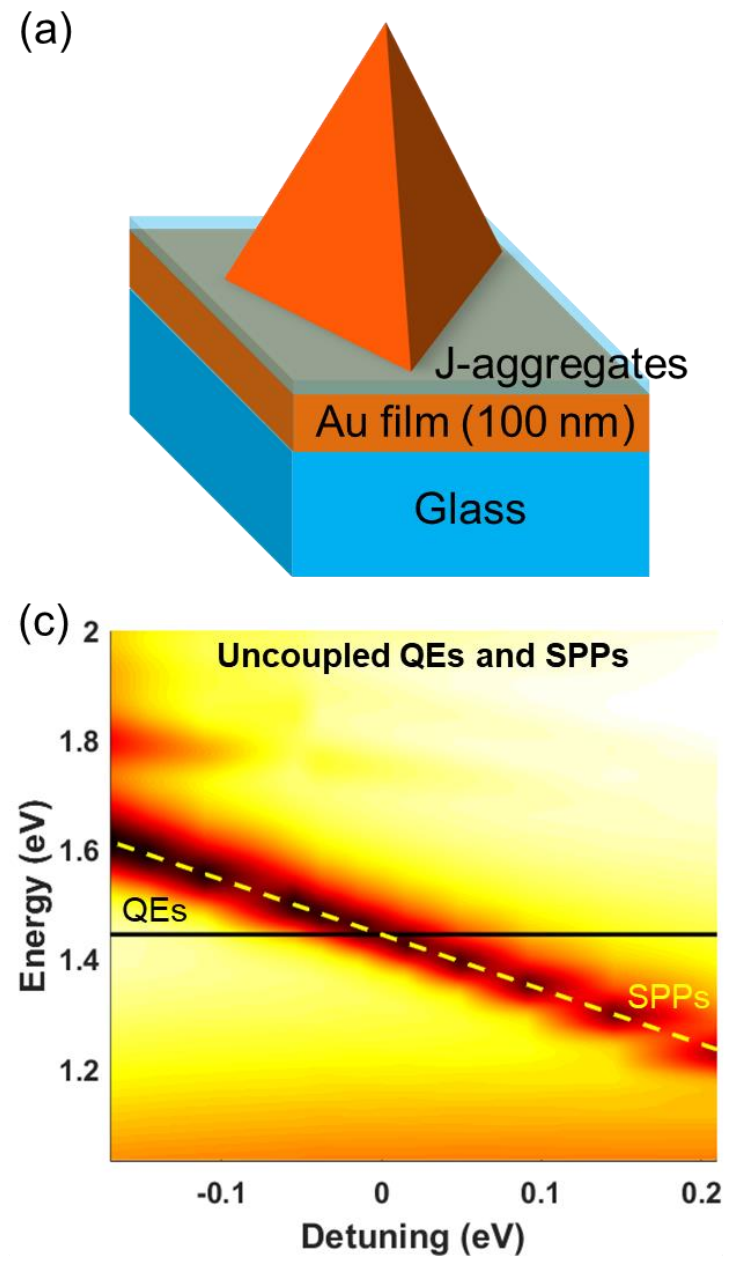
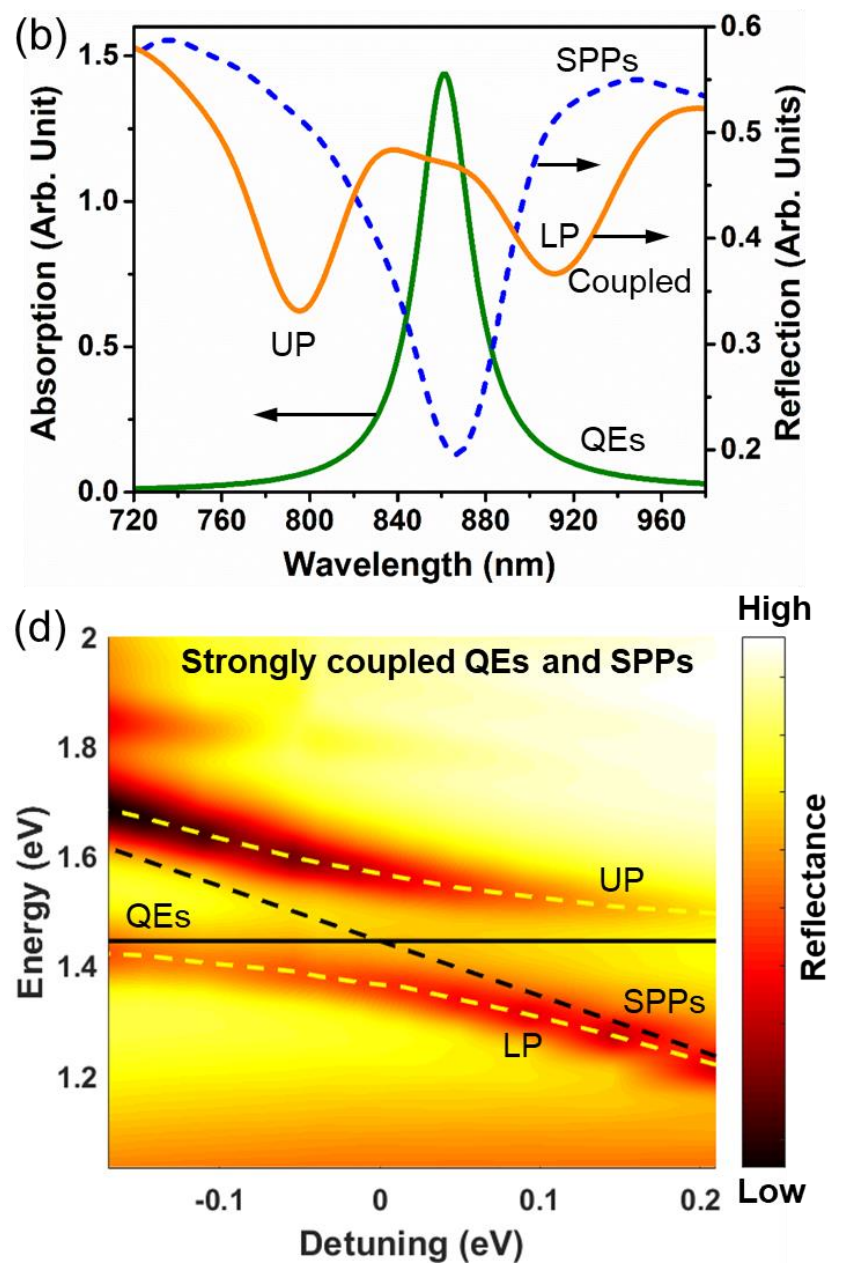

Figure 4.5: (a) Scheme of the film-coupled gold nanopyramid array structure with a layer of thickness-varying $J$ aggregates sandwiched at the spacer layer; (b) Spectral profiles of $J$ aggregates, SPPs and the hybridized upper (UP) and lower $(L P)$ polaritonic states (c) Dispersion of SPPs compared to the absorption line of QEs when they were uncoupled; (d) Dispersion of the hybridized polaritonic states (UP and LP) generated from the strongly-coupled SPPs-QEs system

Strong coupling. The capability of the fabricated film-coupled Au nanopyramid array to reach the strong-coupling regime when coupled with an excitonic material was studied numerically by FDTD simulations. Instead of silica, J-aggregates were modelled as QEs in the spacer layer, as shown in Figure 4.5(a). The permittivity of J-aggregates was assumed to follow the Lorentz Model, which was given $b y^{21}$ 


$$
\varepsilon(\omega)=\varepsilon_{\infty}+\frac{f_{0} \omega_{0}^{2}}{\omega_{0}^{2}-\omega^{2}-i \gamma_{0} \omega}
$$

The absorption peak of J-aggregates was assumed at $861 \mathrm{~nm}\left(\omega_{0}=1.44 \mathrm{eV}\right)$; the damping rate was assumed as $\gamma_{0}=50 \mathrm{meV}$; the high-frequency permittivity $\varepsilon_{\infty}$ and Lorentz permittivity $f_{0}$ were set as 1.45 and $50 \mathrm{meV}$, respectively.

The absorption spectrum of J-aggregates was derived from the imaginary part of the complex permittivity given by Equation (1) and was shown in Figure 4.5(b). In the meanwhile, the reflection spectra for the corresponding plasmonic gap modes were calculated using FDTD simulations by modelling the spacer layer as an insulator with a fixed refractive index of 1.25 , roughly the mean value of the real part of the permittivity of J-aggregates.

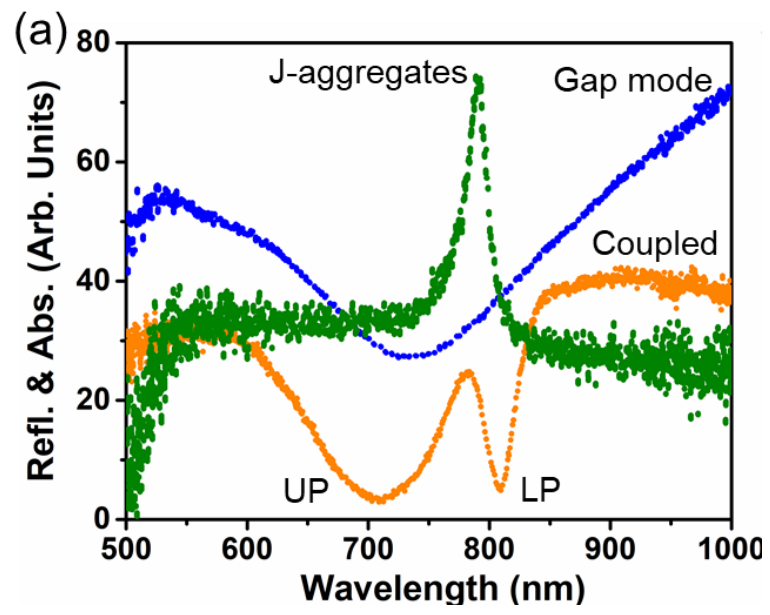

Figure 4. 6: (a) Experimentally measured spectral profiles of J-aggregates (absorption, olive curves), plasmonic gap modes (reflection, blue curves), and the hybridized upper (UP) and lower $(L P)$ plexciton states (reflection, orange curves) when a layer of J-aggregates were sandwiched at the spacer layer

Without coupling, the dispersion of SPPs was independent of the absorption line of QEs, as shown in Figure 4.5(c), where the detuning was defined as the energy difference between SPPs and QEs. With a layer of $16 \mathrm{~nm}$ J-aggregates filled into the spacer, SPPs and QEs were spectrally mixed. Instead of being independent of each other, their spectral mixture led to the Rabi splitting of the plasmonic gap mode and generated two new hybridized modes at $795 \mathrm{~nm}$ and $912 \mathrm{~nm}$, respectively, as shown in Figure 4.5(b). The mode splitting, however, does not necessarily guarantee that the strong-coupling regime is reached. As it has been well documented previously, 
the plasmonic coupling and Fano resonance could also cause similar features. ${ }^{37-38}$ A positive confirmation of strong coupling requires that the coupling strength far exceed any other damping rates.

For the case with a layer of $16 \mathrm{~nm} \mathrm{J-aggregates} \mathrm{sandwiched} \mathrm{in} \mathrm{the} \mathrm{film-coupled} \mathrm{gold}$ nanopyramid array, the Rabi frequency was calculated as $\Omega_{R}=200 \mathrm{meV}$. The coupling strength

was found as $g=\frac{1}{2} \Omega_{R}=100 \mathrm{meV}$. The damping rate of SPPs was obtained by fitting the reflection dip with a Lorentzian function, which was given as $\kappa=56 \mathrm{meV}$. The fact that $g>\kappa, \gamma_{0}$ confirms that strong coupling was indeed achieved in this nanostructure. The two new hybridized modes were actually two newly formed polaritonic states corresponding to upper polariton (UP) and lower polariton (LP), which are half-light, half matter. These entangled states are characteristic of strong coupling where SPPs and J-aggregates lose their own identities. The confined energy was rapidly and coherently exchanged between SPPs and J-aggregates at a rate of the Rabi frequency. By varying the thickness of the J-aggregate layer, the anti-crossing behavior of the two polaritonic states were observed, as shown in Figure 4.5(d), which would not occur in the weakcoupling regime. ${ }^{10}$

The anti-crossing region is of great fundamental significance. It has been predicted to give rise to numerous intriguing optical effects such as photon blockade, emission of correlated photons, nonclassical or squeezed state generation, super-radiant phase transitions, and ultra-efficient light emission. ${ }^{10,39}$ Furthermore, the problems of a high energy loss in metallic nanostructures can be effectively circumvented since the rates of any other damping channels are outcompeted by the overwhelmingly strong coupling rate in the strong-coupling region. The demonstration of reaching the strong-coupling regime by sandwiching a layer of excitonic material in the film-coupled gold nanopyramid array has strong implications in designing state-of-the-art optoelectronic devices with new functionalities by fully utilizing the nonlinearity of QEs and the transportability of SPPs.

\subsection{CONCULUSION}

In summary, a long-range ordered gold nanopyramid array pattern was fabricated on the glass substrate by nanosphere lithography. The gold nanopyramid array exhibited strong light scattering, displaying an intense reflection peak. The maximum electric field enhancement $(|\mathbf{E}| /|\mathbf{E} 0|)$ was 
estimated to be 271 at the corners, which decayed exponentially away from the pyramid bottom. After the gold nanopyramid array was coupled to a gold film, a plasmonic gap mode was generated in the silica spacer layer, which led to strong light absorption confined in the spacer layer. In case of a $10 \mathrm{~nm}$ thick spacer layer, the maximum electric field enhancement $\left(|\mathbf{E}| /\left|\mathbf{E}_{0}\right|\right)$ and the magnetic field enhancement $\left(|\mathbf{H} /| \mathbf{H}_{0} \mid\right)$ were estimated to be 233 and 25, respectively, which occurred at the gap edge. The magnetic field remained almost constant and the electric field decayed much slower with an increase in the distance away from the pyramid bottom. Because of the induced charges on opposite sides of the gap, the confined EM energy was found to oscillate back and forth between the spatially separated electric and magnetic counterparts, which made the effective mode volume to be approximately the actual volume of the gap region. By filling the gap with J-aggregates, strong coupling between the plasmonic gap mode and J-aggregates was demonstrated. The demonstration of strong coupling on this two-dimensional metallic nanostructure not only effectively circumvents the unavoidable high loss on metals, but also makes it possible to fully utilize the nonlinearity of QEs and the high transportability of SPPs in designing state-of-the-art optoelectronic devices. 


\section{References}

[1]. Nocera, D. G. The Artificial Leaf. Accounts Chem Res 2012, 45, 767-776.

[2]. Hochbaum, A. I.; Yang, P. D. Semiconductor Nanowires for Energy Conversion. Chem Rev 2010, 110, 527-546.

[3]. Anker, J. N.; Hall, W. P.; Lyandres, O.; Shah, N. C.; Zhao, J.; Van Duyne, R. P. Biosensing with plasmonic nanosensors. Nat Mater 2008, 7, 442-453.

[4]. Grotjohann, T.; Testa, I.; Leutenegger, M.; Bock, H.; Urban, N. T.; Lavoie-Cardinal, F.; Willig, K. I.; Eggeling, C.; Jakobs, S.; Hell, S. W. Diffraction-unlimited all-optical imaging and writing with a photochromic GFP. Nature 2011, 478, 204-208.

[5]. Hugall, J. T.; Singh, A.; van Hulst, N. F. Plasmonic Cavity Coupling. Acs Photonics 2018, 5, 43-53.

[6]. Maier, S. A., Plasmonics: Fundamentals and Applications. Springer US: 2010.

[7]. Stockman, M. I. Spasers explained. Nat Photonics 2008, 2, 327-329.

[8]. Kolesov, R.; Grotz, B.; Balasubramanian, G.; Stohr, R. J.; Nicolet, A. A. L.; Hemmer, P. R.; Jelezko, F.; Wrachtrup, J. Wave-particle duality of single surface plasmon polaritons. Nat Phys 2009, 5, 470-474.

[9]. MacDonald, K. F.; Samson, Z. L.; Stockman, M. I.; Zheludev, N. I. Ultrafast active plasmonics. Nat Photonics 2009, 3, 55-58.

[10]. Vasa, P.; Lienau, C. Strong Light-Matter Interaction in Quantum Emitter/Metal Hybrid Nanostructures. Acs Photonics 2018, 5, 2-23.

[11]. Maser, A.; Gmeiner, B.; Utikal, T.; Gotzinger, S.; Sandoghdar, V. Few-photon coherent nonlinear optics with a single molecule. Nat Photonics 2016, 10, 450-453.

[12]. Pfeiffer, M.; Atkinson, P.; Rastelli, A.; Schmidt, O. G.; Giessen, H.; Lippitz, M.; Lindfors, K. Coupling a single solid-state quantum emitter to an array of resonant plasmonic antennas. Sci Rep-Uk 2018, 8.

[13]. Klimov, V. I.; Mikhailovsky, A. A.; Xu, S.; Malko, A.; Hollingsworth, J. A.; Leatherdale, C. A.; Eisler, H. J.; Bawendi, M. G. Optical gain and stimulated emission in nanocrystal quantum dots. Science 2000, 290, 314-317.

[14]. Zhang, P.; Protsenko, I.; Sandoghdar, V.; Chen, X. W. A Single-Emitter Gain Medium for Bright Coherent Radiation from a Plasmonic Nanoresonator. Acs Photonics 2017, 4, 2738-2744. 
[15]. Zhou, Y.; Wang, Z.; Rasmita, A.; Kim, S.; Berhane, A.; Bodrog, Z.; Adamo, G.; Gali, A.; Aharonovich, I.; Gao, W.-b. Room temperature solid-state quantum emitters in the telecom range. Science Advances 2018, 4.

[16]. Lichtmannecker, S.; Florian, M.; Reichert, T.; Blauth, M.; Bichler, M.; Jahnke, F.; Finley, J. J.; Gies, C.; Kaniber, M. A few-emitter solid-state multi-exciton laser. Sci Rep-Uk 2017, 7.

[17]. Chikkaraddy, R.; de Nijs, B.; Benz, F.; Barrow, S. J.; Scherman, O. A.; Rosta, E.; Demetriadou, A.; Fox, P.; Hess, O.; Baumberg, J. J. Single-molecule strong coupling at room temperature in plasmonic nanocavities. Nature 2016, 535, 127-130.

[18]. Gross, H.; Hamm, J. M.; Tufarelli, T.; Hess, O.; Hecht, B. Near-field strong coupling of single quantum dots. Science Advances 2018, 4.

[19]. Chevrier, K.; Benoit, J. M.; Symonds, C.; Paparone, J.; Laverdant, J.; Bellessa, J. Organic Exciton in Strong Coupling with Long-Range Surface Plasmons and Waveguided Modes. Acs Photonics 2018, 5, 80-84.

[20]. Eizner, E.; Avayu, O.; Ditcovski, R.; Ellenbogen, T. Aluminum Nanoantenna Complexes for Strong Coupling between Excitons and Localized Surface Plasmons. Nano Lett 2015, 15, 62156221.

[21]. Zengin, G.; Johansson, G.; Johansson, P.; Antosiewicz, T. J.; Kall, M.; Shegai, T. Approaching the strong coupling limit in single plasmonic nanorods interacting with $\mathrm{J}$-aggregates. Sci Rep-Uk 2013, 3.

[22]. Torma, P.; Barnes, W. L. Strong coupling between surface plasmon polaritons and emitters: a review. Rep Prog Phys 2015, 78.

[23]. Wang, H.; Wang, H. Y.; Bozzola, A.; Toma, A.; Panaro, S.; Raja, W.; Alabastri, A.; Wang, L.; Chen, Q. D.; Xu, H. L.; De Angelis, F.; Sun, H. B.; Zaccaria, R. P. Dynamics of Strong Coupling between J-Aggregates and Surface Plasmon Polaritons in Subwavelength Hole Arrays. Adv Funct Mater 2016, 26, 6198-6205.

[24]. Zengin, G.; Wersall, M.; Nilsson, S.; Antosiewicz, T. J.; Kall, M.; Shegai, T. Realizing Strong Light-Matter Interactions between Single-Nanoparticle Plasmons and Molecular Excitons at Ambient Conditions. Phys Rev Lett 2015, 114.

[25]. Liu, R. M.; Zhou, Z. K.; Yu, Y. C.; Zhang, T. W.; Wang, H.; Liu, G. H.; Wei, Y. M.; Chen, H. J.; Wang, X. H. Strong Light-Matter Interactions in Single Open Plasmonic Nanocavities at the Quantum Optics Limit. Phys Rev Lett 2017, 118. 
[26]. Baranov, D. G.; Wersall, M.; Cuadra, J.; Antosiewicz, T. J.; Shegai, T. Novel Nanostructures and Materials for Strong Light Matter Interactions. Acs Photonics 2018, 5, 24-42.

[27]. Vasa, P.; Pomraenke, R.; Cirmi, G.; De Re, E.; Wang, W.; Schwieger, S.; Leipold, D.; Runge, E.; Cerullo, G.; Lienau, C. Ultrafast Manipulation of Strong Coupling in Metal-Molecular Aggregate Hybrid Nanostructures. Acs Nano 2010, 4, 7559-7565.

[28]. Chai, Z.; Hu, X.; Wang, F.; Niu, X.; Xie, J.; Gong, Q. Ultrafast All-Optical Switching. Advanced Optical Materials 2017, 5, 1600665.

[29]. Walker, P. M.; Tinkler, L.; Skryabin, D. V.; Yulin, A.; Royall, B.; Farrer, I.; Ritchie, D. A.; Skolnick, M. S.; Krizhanovskii, D. N. Ultra-low-power hybrid light-matter solitons. Nat Commun 2015,6 .

[30]. Mortensen, N. A.; Raza, S.; Wubs, M.; Sondergaard, T.; Bozhevolnyi, S. I. A generalized non-local optical response theory for plasmonic nanostructures. Nat Commun 2014, 5.

[31]. Esteban, R.; Borisov, A. G.; Nordlander, P.; Aizpurua, J. Bridging quantum and classical plasmonics with a quantum-corrected model. Nat Commun 2012, 3.

[32]. Jurga, R.; D'Agostino, S.; Della Sala, F.; Ciraci, C. Plasmonic Nonlocal Response Effects on Dipole Decay Dynamics in the Weak- and Strong-Coupling Regimes. J Phys Chem C 2017, 121, 22361-22368.

[33]. Zhu, W. Q.; Esteban, R.; Borisov, A. G.; Baumberg, J. J.; Nordlander, P.; Lezec, H. J.; Aizpurua, J.; Crozier, K. B. Quantum mechanical effects in plasmonic structures with subnanometre gaps. Nat Commun 2016, 7.

[34]. Santhosh, K.; Bitton, O.; Chuntonov, L.; Haran, G. Vacuum Rabi splitting in a plasmonic cavity at the single quantum emitter limit. Nat Commun 2016, 7.

[35]. Hoang, T. B.; Akselrod, G. M.; Mikkelsen, M. H. Ultrafast Room-Temperature Single Photon Emission from Quantum Dots Coupled to Plasmonic Nanocavities. Nano Lett 2016, 16, 270-275. [36]. Haynes, C. L.; Van Duyne, R. P. Nanosphere lithography: A versatile nanofabrication tool for studies of size-dependent nanoparticle optics. J Phys Chem B 2001, 105, 5599-5611.

[37]. Prodan, E.; Radloff, C.; Halas, N. J.; Nordlander, P. A hybridization model for the plasmon response of complex nanostructures. Science 2003, 302, 419-422.

[38]. Limonov, M. F.; Rybin, M. V.; Poddubny, A. N.; Kivshar, Y. S. Fano resonances in photonics. Nat Photonics 2017, 11, 543-554. 
[39]. Birnbaum, K. M.; Boca, A.; Miller, R.; Boozer, A. D.; Northup, T. E.; Kimble, H. J. Photon blockade in an optical cavity with one trapped atom. Nature 2005, 436, 87-90.

[40]. Johnson, P. B.; Christy, R. W. Optical Constants of the Noble Metals. Physical Review B $1972,6,4370-4379$. 


\section{CHAPTER 5: ENHANCED FLUORESCENCE OF METAL ORGANIC FRAMEWORKS IN PLASMONIC GAP MODE}

Fluorescence based biosensors have become a dominant and most efficient tool for biosensing. Metal Enhanced Fluorescence is a phenomenon where the intensity of fluorescent material signal is enhanced several orders of magnitude, when fluorophore is placed in the vicinity of plasmonic nanostructure. Among all the plasmonic modes, gap mode has large field enhancement and mode volume with high Purcell factor. In this chapter, MOF fluorescent signal is enhanced in gap mode generated by pyramids on gold film 3D nanostructure. The device is designed and optimized based on the spectral overlap of gap mode on to the fluorescent emission for maximizing the Purcell factor.

\subsection{INTRODUCTION}

Metal nanostructures have showed unique optical properties which were used in a wide variety of applications $^{1-5}$ like bio-sensing, solar energy, photonic crystals etc. Particularly plasmon enhanced spectroscopies like Surface Enhanced Raman Scattering (SERS) ${ }^{6-8}$ and Metal Enhanced Fluorescence $(\mathrm{MEF})^{9-15}$ were become dominant techniques in bio-sensing and medical diagnostics. Plasmonic structures localize the optical energy into the nanoscale features in the form of EM field energy. This plasmonic property is known as localized surface plasmon resonance (LSPR), which arise from the collective oscillation of free electrons with the incident light. LSPR of a nanostructure depends on the size, shape, dielectric environment and coupling between adjacent nanostructures.

Quick and efficient detection of trace physiological markers and chemicals need to be detected for health and food safety. For this reason, biosensors are being rapidly developed. Fluorescence technique is used to detect DNA microarrays and bio molecules by attaching fluorophores. However, low concentration of biomolecules need to be enhanced several times for the detection. Recently, MEF is emerging as a new tool for enhancing the fluorescence signal by using metal 
particles. The increase in the fluorescence emission of fluorophore in the vicinity of EM filed of metal nano particle is MEF phenomenon. The stronger interaction occurring between fluorophore excited states and the EM field of metal nano particles increases or decreases the radiative decay rate of fluorophore. MEF has shown significant progress as a tool for fluorescence spectroscopy ${ }^{16}$, because of its unique features like sensitivity, ease of use and higher cross-section. MEF is extensively studied for different shape, size and separation between nanoparticles. However, it is an experimental challenge to design a MEF based nano structure on a large scale. There are three major factors that affect the efficiency of MEF a) The size and type of metal nanoparticle b) emission wavelength of fluorophore c) Intrinsic quantum yield of fluorophore. For metal nano particles less than 40nm generally absorb light quenches the fluorescence emission. Similarly, nano particles greater than $40 \mathrm{~nm}$, scatters light which enhances the fluorescence emission. In addition to size dependency there is another most efficient way to enhance the fluorescence. When there is a spectral overlap between the fluorescence emission with the surface plasmon resonance there is a non-radiative energy transfer from fluorescent species to the metal nano particles. 


\section{a) Silicon cleaning}

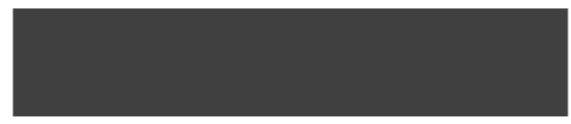

b) $100 \mathrm{~nm}$ gold deposition

e) Titanium deposition

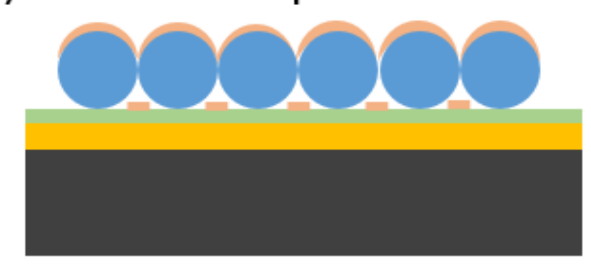

f) Silver deposition

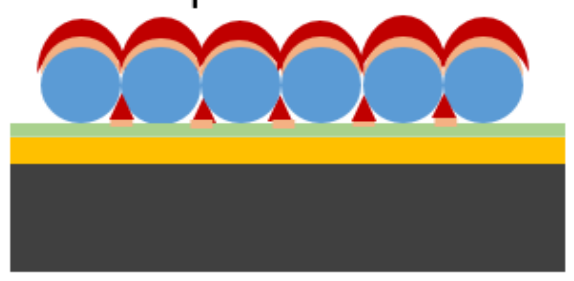

g) Removal of polystyrene beads

c) MOF layer by layer deposition

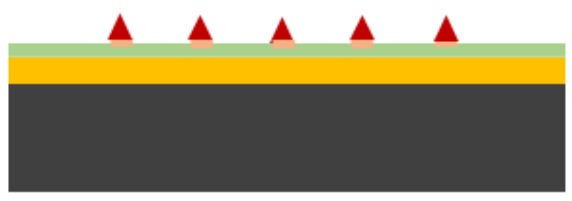

h) Schematic silver nanopyramid array

d) Dip coating of polystyrene beads
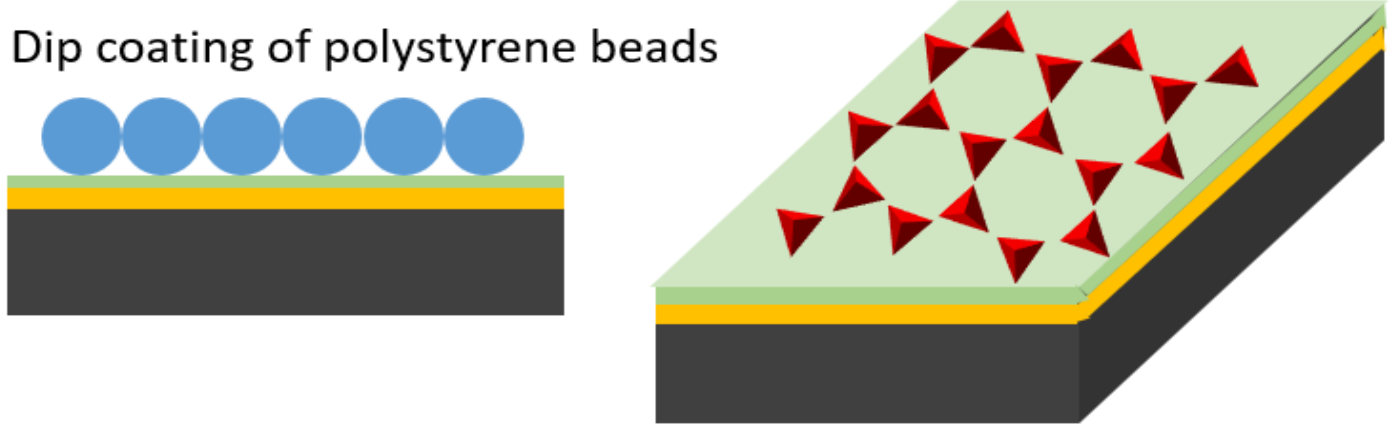

Figure 5.1: Nanosphere lithography fabrication of silver nanopyramids coupled gold film for exciting gap plasmon in the MOF spacer layer

In this chapter, gap surface plasmon ${ }^{17-20}$ mode is generated from coupling silver nano pyramids and gold film which are separated by a spacer layer. Nano Sphere Lithography is used for large area and uniform array fabrication of silver nano pyramid structure. A uniform layer of MOF, which is a fluorescent material is used as a spacer layer. The gap plasmon mode is confined in the spacer layer which is MOF. Thus fluorescent material is in vicinity of the plasmon mode. In this regard, there is a 2 orders of enhancement of MOF fluorescent signal in gap plasmon mode. 


\subsection{Methods}

\section{Chemicals and Materials}

Gold-coated glass slides were purchased from Fisher Scientific Inc. Polystyrene microspheres with a diameter of $500 \mathrm{~nm}$ were purchased from Thermo Scientific (Waltham, MA). MOF synthesis process is giving after this section. Acetone, methanol, and sulphuric acid (H2SO4) and hydrogen peroxide were purchased from Alfa Aesar (Haverhill, MA). Deionized (D.I.) water was produced by the Milli-Q Integral 3/5/10/15 system (18.2 MO cm, Millipore Corp., U.S.A.)

\section{MOF synthesis}

$\mathrm{ZrCl} 4$ (70 mg), TCPP (50 mg) and benzoic acid (2,700 mg) were added into $8 \mathrm{~mL}$ of DEF, ultrasonically dissolved. The mixture was heated in $120{ }^{\circ} \mathrm{C}$ oven for $48 \mathrm{~h}$. After the reaction, red needle shaped crystals were harvested by filtration $(35 \mathrm{mg}, 47 \% \text { yield })^{22,23}$

\section{Instrumentation}

An e-beam evaporator (Kurt J. Lesker) was used to deposit silver. A scanning electron microscope (JEOL-JSM-7600F) was used to characterize the fabricated film-coupled silver nanopyramid array. Reflection spectra were acquired by an Ocean Optics USB 4000 spectrometer and DTMINI-2-GS. Fluorescence spectra measurements were taken using Hoiba Flurolog-3 spectrofluorometer.

\subsection{RESULTS AND DISCUSSIONS}

\section{Fabrication of nanopyramid array coupled gold film}

The step by step fabrication process is shown in Figure 5.1. Initially a silicon chip of roughly $0.5 \mathrm{~cm}$ $\mathrm{x} 0.5 \mathrm{~cm}$ is cut down. The chips are cleaned successively in acetone, methanol and water for $5 \mathrm{~min}$ in each. The chips are dried in $\mathrm{N}_{2}$ gas. $100 \mathrm{~nm}$ gold is deposited using e-beam evaporator on to the silicon chips. Using layer by layer approach MOF is deposited on to the gold surface. For this application 7 monolayers of MOF is deposited. Polystyrene beads are dip coated on to the MOF and dried to form a uniform monolayer of PS film. Later, 5nm of titanium and 200nm of silver is 
deposited using e-beam evaporator. Due to the interstitial gaps in the PS bead, the deposited material takes the shape of pyramids as shown in figure 5.1(h). The chips are ultrasonicated in methanol to remove PS beads and leaving only pyramids. In this process titanium is used as an adhesion layer for silver pyramids to stick to the substrate.

\section{Excitation of gap plasmon mode in silver nanopyramid array coupled gold film}

Silver nanopyramid array-MOF- gold film structure is designed such a way the gap plasmon mode excited in this structure which is used for metal enhanced fluorescence. For this first the gap mode of such MIM structure is tunable over large range to overlap with the emission wavelength of fluorescent material. The MOF is layer by layer deposited as a spacer layer, which is fluorescent material. This fluorophore is sandwiched in between the nanostructures (Figure 5.2(a)) to get the efficient metal enhanced fluorescence.

Initially optical properties were studied to optimize the fluorescence signal enhancement. Figure 5.2 (b) shows the absorption spectra of MOF on gold and the coupled structure. In the absorption spectra of MOF, the band edge is around 550nm which is due to the band gap of MOF. In the sandwich structure, there is absorption peak at $650 \mathrm{~nm}$ this is due to the gap mode formation which strongly absorbs light. The gap plasmon mode is formed due to the coupling between gold film and the silver nanopyramids. The gap plasmon peak is very sensitive to the thickness of the MOF film. Since the maximum enhancement can be achieved when the fluorescent peak of MOF overlaps with the plasmon peak, the plasmon peak is tuned by varying the thickness of MOF film. 
a)
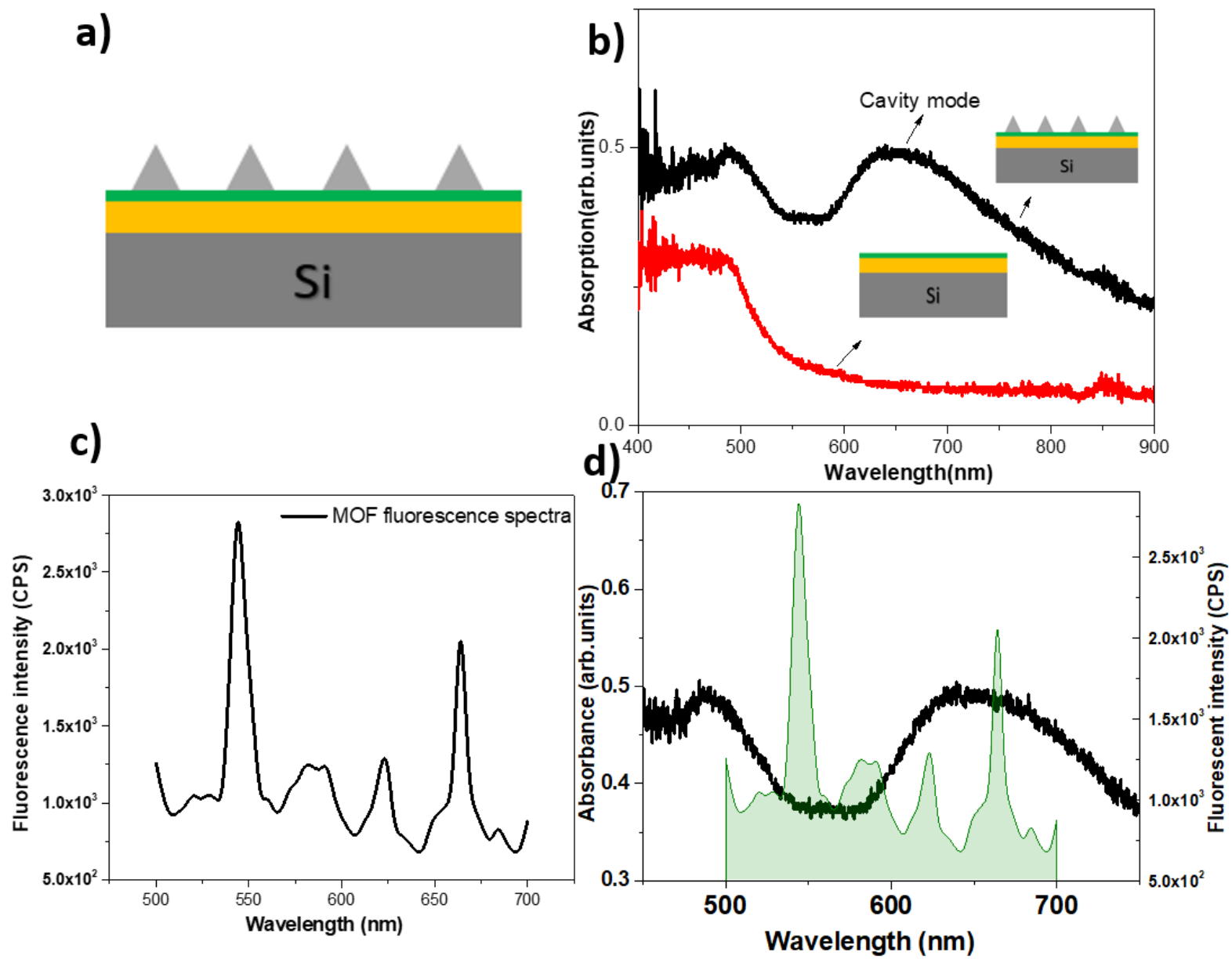

Figure 5. 2: a) Schematic of nanopyramid coupled with gold film with MOF spacer layer b) Absorption spectra of proposed structure with pyramids and without pyramids c) Fluorescence spectra of MOF on glass d) Overlay graph of nanostructure absorption and MOF fluorescence spectra

For 7layers of MOF deposition, the plasmon peak exists at $650 \mathrm{~nm}$ which exactly overlap with the fluorescent peak of MOF shown in figure 5.2 (d). The observed intense absorption peak was ascribed to the plasmonic gap modes, which were induced in the spacer layer between the Ag nanopyramid array and the underlying Au film. Since a transmission line type of resonance can be sustained at the gap ${ }^{21}$ the incident light can be guided into the gap region and adjust itself to satisfy the boundary conditions. The impedance mismatch at the gap edge causes most of the incident light to be trapped inside the gap, and thus contributes to the strong absorption. 


\section{Fluorescent enhancement}

To demonstrate the fluorescence enhancement, the MOF fluorescence spectra is acquired by excitation wavelength $410 \mathrm{~nm}$ as shown in the Figure 5.2 (c). The fluorescent peak at $665 \mathrm{~nm}$ is overlaped with the cavity mode of the sandwich structure for maximum fluorescent enhancement. The coupling of fluorophore with gap resonant mode affects the intensity of fluorescence emission.
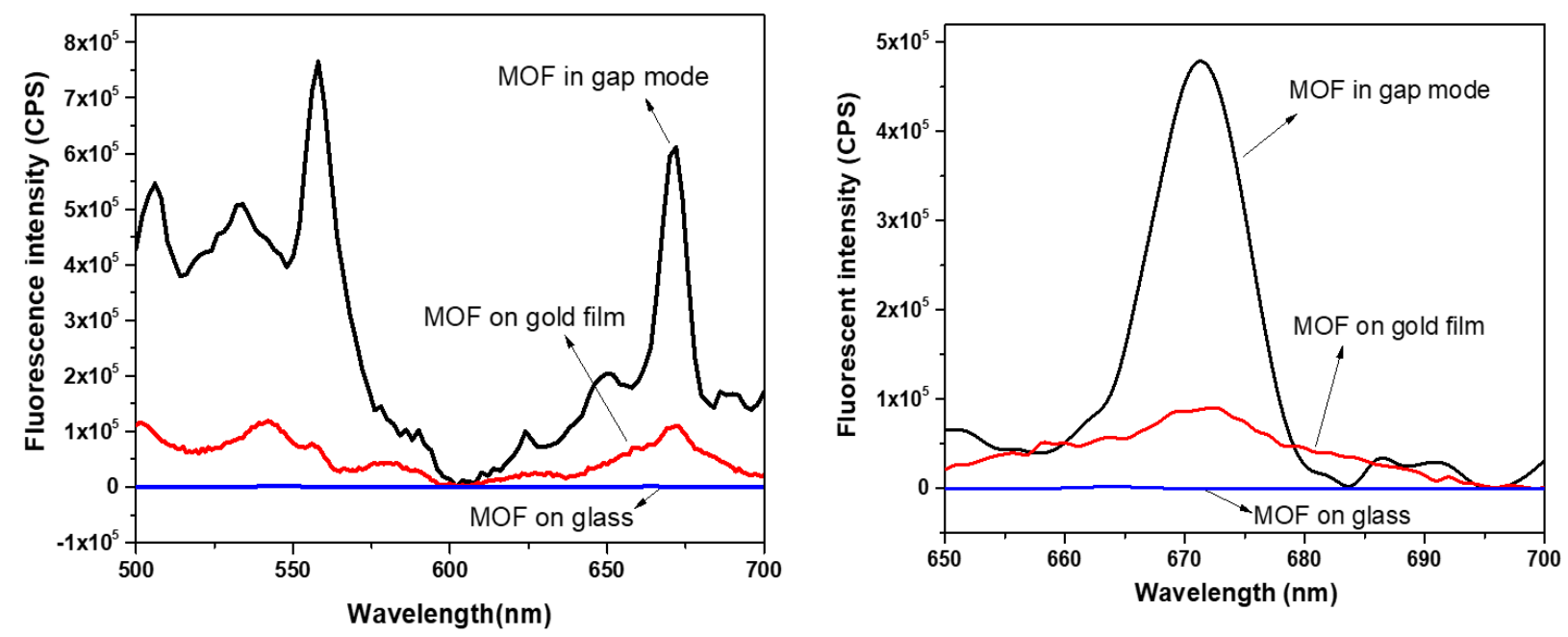

Figure 5. 3: a) Fluorescence spectra comparison b) Fluorescence spectra from 650nm to 700nm range

Figure 5.3 shows the comparison of fluorescence spectra of MOF, MOF on gold film and MOF in gap plasmon mode. There is a distinct enhancement of fluorescence signal of MOF in gap mode compared to the one on glass substrate. The strong enhancement is due to the Electromagnetic field created by the gap mode. electric field was confined within the gap for the film-coupled Au pyramid array while a radial electrical field appeared at the corners for the Ag nanopyramid array. The magnetic field was kept almost constant in the gap for the film-coupled Au pyramid array and the electric field underwent only a small decay with an increase in the distance away from the pyramid bottom along the cross-section. In stark contrast, the electric field from the corner mode of the Ag pyramid array alone decayed exponentially away with an increase in the distance away from the pyramid bottom. Thanks to these unique features, the localized EM energy oscillated back and forth between the electric and magnetic counterparts inside the gap and it only got damped either because of metal loss or by coupling to the free space. The gap which confines the spatially oscillating EM energy thus defines a spectrally tunable plasmonic cavity mode with an 
effective mode volume that is approximately the physical size of the gap. This feature provides an excellent configuration to enable the maximum interaction between SPPs and the MOF in the gap. To assess the performance of sandwich nanostructure in enhancing fluorescence, a peak centered around $670 \mathrm{~nm}$ is considered and base line is calculated. The comparison of fluorescence peak on different substrates is shown in figure 5.3 (b). The fluorescence enhancement factor was found to be $5^{*} 10^{2}$.

\subsection{CONCLUSION}

In summary, a long-range ordered silver nanopyramid array pattern was fabricated on a MOF-Au film by nanosphere lithography. The nanopyramid array was coupled to gold film to form a plasmonic gap mode in MOF spacer layer, which led to strong light absorption. The absorbed light is trapped in the gap (MOF layer) to generate huge EM filed. The gap mode is spectrally tuned to overlap the fluorescent peak of MOF. The fluorescence emission measurements on MOF and MOF in the gap mode demonstrated the enhancement factor around $5^{*} 10^{2}$, which can be used in MEF based biosensing applications. The gap plasmon is more effective for enhanced fluorescence because of the huge EM filed generated in the cavity. 


\section{References}

[1] Rao, C.N.R., Kulkarni, G.U., Thomas, P.J. and Edwards, P.P. Size Dependent Chemistry: Properties of Nanocrystals. Chemistry A, 2002, 8, 28-35

[2] Stockman, M.I. Nanoplasmonics: The Physics behind the Application. Physics Today,2011, 64, 39-44

[3] Kirill zilberberg, Thomas Riedl. Metal-nanostructures - a modern and powerful platform to create transparent electrodes for thin-film photovoltaics, J. Mater. Chem. A, 2016,4, 14481-14508 [4] Suljo Linic, Phillip Christopher, David Ingram, Plasmonic-metal nanostructures for efficient conversion of solar to chemical energy, Nature Materials, 2011, 10, 911-921.

[5] Stefan A. Maier, Plasmonics: Metal Nanostructures for Subwavelength Photonic Devices, IEEE Journal of Selected Topics in Quantum Electronics, 2006, 12, 6,1214-1220, 2006.

[6] Vo-Dinh T., Yan F., Stokes D.L. Plasmonics-Based Nanostructures for Surface-Enhanced Raman Scattering Bioanalysis. Protein Nanotechnology. Methods in Molecular Biology (2005), 300.

[7] Fleischmann, M., Hendra, P. J., and McQuillan, A. J. Raman-spectra of pyridine adsorbed at a silver electrode. Chem. Phys. Lett. 1974, 26, 163-166

[8] Jeanmaire, D. L. and Van Duyne, R. P. Surface Raman spectro-electrochemistry. 1. Heterocyclic, aromatic, and aliphatic-amines adsorbed on anodized silver electrode. J. Electroanal. Chem. 1977, 84, 1-20

[9] Fothergill S M, Metal enhanced fluorescence biosensing: from ultra-violet towards second near-infrared window, Nanoscale, 2018, 10, 20914.

[10] Zhu Z. Plasmon-Enhanced Fluorescence in Coupled Nanostructures and Applications in DNA Detection, ACS Appl. Bio Mater. 2018, 1, 118-124

[11] Klantsataya E. Effect of surface roughness on metal enhanced fluorescence in planar substrates and optical fibers, optical materials express, 2016, 6, 2128.

[12] Abel B. Metal-Enhanced Fluorescence from Silver Nanowires with High Aspect Ratio on Glass Slides for Biosensing Applications, J. Phys. Chem. C 2015, 119, 675-684

[13] Jeong Y. Metal enhanced fluorescence (MEF) for biosensors: General approaches and a review of recent developments, Biosensors and Bioelectronics 111 (2018) 102-116

[14] Bauch M, Toma K. Plasmon-Enhanced Fluorescence Biosensors: a Review, Plasmonics (2014) 9, 781-799. 
[15] Abdulhalim, I., Karabchevsky, A., Patzig, C., Rauschenbach, B., Fuhrmann, B., Eltzov, E., Marks, R., Xu, J., Zhang, F. and Lakhtakia, A.Surface-enhanced fluorescence from metal sculptured thin films with application to biosensing in water. Applied Physics Letters, 2009, 94(6), p.063106.

[17] Schmelzeisen M, Zhao Y, Klapper M. Fluorescence Enhancement from Individual Plasmonic Gap Resonances, ACS NANO, 2010, 4, 6,3309-3317.

[18] Sugimoto H. Hybridized Plasmonic Gap Mode of Gold Nanorod on Mirror Nanoantenna for Spectrally Tailored Fluorescence Enhancement, ACS Photonics 2018, 5, 3421-3427

[19] Wu Z, Yang J. Gap-Mode Surface-Plasmon-Enhanced Photoluminescence and Photoresponse of MoS2, Adv. Mater. 2018, 30, 1706527.

[20] Zheng P, Kasani S. Converting plasmonic light scattering to confined light absorption and creating plexcitons by coupling a gold nano-pyramid array onto a silica-gold film, Nanoscale Horiz., 2019, 4, 516.

[21] Zangwill A. Modern Electrodynamics, Cambridge University Press, 2013.

[22] Yang $\mathrm{H}$ et.al, Metal-organic framework coated titanium dioxide nanorod array $\mathrm{p}-\mathrm{n}$ heterojunction photoanode for solar water-splitting, Nano Res.2019, 12(3): 643-650

[23] Jiang, H. L et.al, An exceptionally stable, porphyrinic Zr metal-organic framework exhibiting pH-dependent fluorescence. J. Am. Chem. Soc. 2013, 135, 13934-13938. 


\section{CHAPTER 6: INEXPENSIVE SEMICONDUCTOR MATERIALS FOR PLASMONIC APPLICATIONS}

Two dimensional semiconductor materials have shown plasmon resonance in visible region, having lot of applications in sensing, medicine and photonics. Unlike metals, the plasmon tunability in semiconductors depends on experimental parameters of the material synthesis process. This makes the tuning of plasmonic resonance in semiconductor materials challenging. Here in, the molybdenum oxide thin films were directly deposited and nitrogen annealed which showed a tunable localized surface plasmon resonance (LSPR). A chip based 2D semiconductor material is fabricated to study the structural and size dependent plasmon resonance. This work establishes a way to fabricate chip based ordered semiconductor nanostructures, which helps in systematic study of plasmon properties on nanostructures.

\subsection{INTRODUCTION}

Surface plasmons allow near-field absorption and far-field scattering spectral profiles to be delicately engineered in a diffraction-unlimited manner by either fabricating heterogeneous nanoparticles or 2D nanostructures. They have laid the fundamental framework for surfaceenhanced spectroscopies as well as plasmon-induced resonant energy transfer, and thus have met with huge successes in medical diagnostics ${ }^{1,2,3}$, environmental protection ${ }^{4}$, photonics $^{5,6}$ and energy harvesting $^{7}$ in recent years. Yet, candidate metals supporting strong surface plasmons are restricted to gold and silver given the large energy loss rate in a vast majority of metals. There is an unmet need to identify alternative plasmonic materials without compromising the performance.

Semiconductor nanocrystals (NCs) like metal chalcogenides, metal oxides, metal nitrides and heavily doped semiconductors were investigated for plasmonics ${ }^{33-42,9-14,8,27,60,61}$. In metal chalcogenides like $\mathrm{CuS}$, copper deficiency causes vacancies in the highest energy states in valance band, generating more holes responsible for plasmon resonance ${ }^{43,16}$. In transition metal oxides $^{15,24,25,44}$ outer d orbital valence electrons contribute to the plasmon resonance. Whereas, in the degenerately doped semiconductors ${ }^{26,45,46,41,61}$ the free carriers are tuned by doping concentration to excite plasmon. In all the above semiconductor NCs, generation of free excess 
carriers either by creating vacancies or doping is the reason for plasmon excitation. Inspite of metals having high density of free carriers which generate intense plasmon resonance, there are many advantages of plasmon resonance in semiconductors compared to metals. Since the carrier density in metals is constant, LSPR in metals is tuned by shape, size and refractive index of surroundings. This restrict plasmon resonance tuning $47,48,49,50,51$ over a narrow wavelength range and need to choose a different metal to exceed the rage of application. In contrast, LSPR in plasmonic semiconductors can be tuned with parameters like carrier density, annealing temperature and doping. This gives an advantage of tuning the plasmon peak over a broad range including visible, Near Infrared (NIR) and Infrared(IR) regions. This is because for semiconductor materials the carrier concentration can be varied over a large range $e^{27,52,53,54}\left(10^{19}-10^{21} \mathrm{~cm}^{-3}\right)$. Among semiconductor NCs, transitional metal oxides are unique especially molybdenum trioxide $\left(\mathrm{MoO}_{3}\right) . \mathrm{MoO}_{3}$ is wide bandgap semiconductor $(3.2 \mathrm{eV})^{67}$, and has a stable orthorhombic $\alpha-\mathrm{MoO}_{3}$ phase in layered structure with distorted $\mathrm{MoO}_{6}$ octahedra ${ }^{68}$. High density of free carriers are possible in $\mathrm{MoO}_{3}$ not only because of outer-d valence electrons ${ }^{73}$ but also due the formation of lattice vacancies during the intercalation of $\mathrm{H}^{+}$ions ${ }^{71}$ (reduction of Mo oxidation states). These vacancies in the $\mathrm{MoO}_{\mathrm{x}}$ crystal results in unique properties of plasma tuning, varied electrical conductivity and band gap ${ }^{69,70}$. Many research groups have showed potential applications of plasmonic semiconductors in recent years. Transition metal oxides $\mathrm{W}_{18} \mathrm{O}_{49}$ and $\mathrm{MoO}_{3-\mathrm{x}}$ were able to generate SERS enhancement factor of $3.4 * 10^{4}$ and $1.42 * 10^{4}$ respectively ${ }^{17,14}$, having sensitivities comparable to the noble metal SERS substrates. Visual calorimetric molecular computing system was demonstrated with molybdenum oxide based on plasmonic switching ${ }^{18}$. Plasmonic $\mathrm{MoO}_{3-\mathrm{x}}$ nanosheets with large surface area is used as catalyst to enhance dehydrogenation activity of ammonia boarane ${ }^{19}$. There are other applications in nano photonics, electrocatalysis and microelectronic devices ${ }^{28,29,30}$.

The understanding of plasmon resonance fundamentally in semiconductor NCs and their potential applications demanded development in the field of plasmonic semiconductor NCs. With the development of wet chemical synthesis methods, NC synthesis has become controllable and flexible to tune LSPR properties. There are many wet chemical synthesis methods like solvothermal method, hydrothermal method, chemical precipitation and colloidal NC synthesis metod to control the size, shape, phase and composition ${ }^{55-59,62-64}$ of NCs. However, the structures produced are highly random and cannot be repeated, which is highly difficult to reproduce. 
Moreover, the fundamental relation of EM field intensity with carrier concentration, morphology and doping is weakly understood in semiconductor NCs. Also, the study on amorphous $\mathrm{MoO}_{3}$, on plasmon resonance is limited by fabrication and preparation methods. This study can be possible if the amorphous $\mathrm{MoO}_{3}$ structures are fabricated repeatedly and in controlled fashion. Zhou et.al demonstrated an ordered mesoporous $\mathrm{WO}_{3}$ using solvent evaporation induced self-assembly method with large surface area ${ }^{65,66}$. However, ordered amorphous molybdenum oxide structures on large scale is difficult to synthesis as anisotropic crystal growth preferentially forms nanosheets and determines the morphology ${ }^{14,19}$. In this paper, chip based plasmonic $\mathrm{MoO}_{3-\mathrm{x}}$ amorphous thin films were fabricated to study the plasmonic behavior under visible light. The films were deposited (physical vapor deposition) and annealed in $\mathrm{N}_{2}$ atmosphere to change the oxygen composition in molybdenum oxide films. The LSPR of Nitrogen treated $\mathrm{MoO} 3$ is tuned across visible to NIR by varying $\mathrm{N}_{2}$ treatment time and annealing temperature, which play a crucial role in the LSPR response intensity. We analyzed the morphological, temperature and duration parameters of $\mathrm{MoO}_{3-\mathrm{x}}$ on plasmon peak.

\section{CHIP BASED PLASMONIC MoO3 FILMS FABRICATION}

(a)

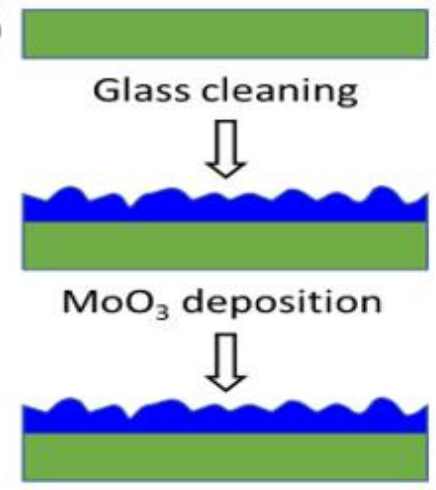

$\mathrm{N}_{2}$ annealing (b)

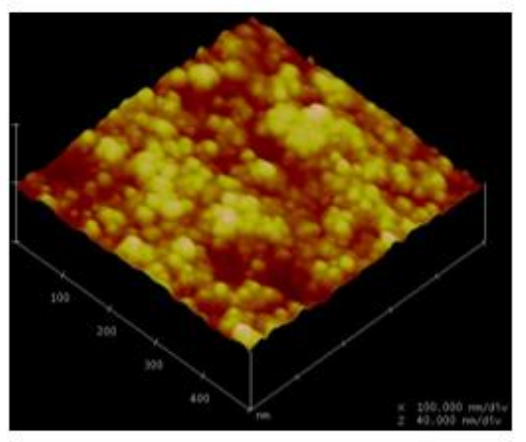

(c)

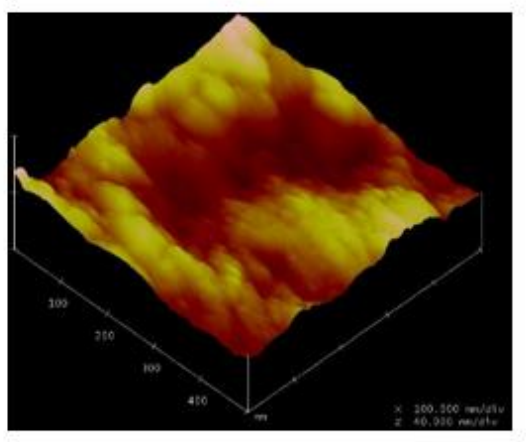

Figure 6. 1: (a) Scheme for MoO3-x thin film fabrication; AFM micrograph images of MoO3-x thin film for (b) as-deposited and (c) annealed sample at $400{ }^{\circ} \mathrm{C}$

The $\mathrm{MoO}_{3-\mathrm{x}}$ thin film was fabricated by a simple two-step process: deposition of molybdenum oxide thin films and annealing in nitrogen atmosphere, as shown in (Figure 6.1(a)). Detailed 
experimental procedures are described in the Methods Section. The AFM micrograph of asdeposited $\mathrm{MoO}_{3-\mathrm{x}}$ films (Figure 6.1(b)) showed uniform and small grains with root mean square (RMS) roughness is around $2 \mathrm{~nm}$. This reflects the amorphous and smooth surface nature of the films. When the films are heated at $400{ }^{\circ} \mathrm{C}$ under $\mathrm{N}_{2}$, the size of the grains increased, with a corresponding increase in RMS surface roughness to around 35nm as shown in the micrograph AFM image in (Figure 6.1(c)). This behavior is due to the agglomeration of smaller particles to form a larger particle with increase in the temperature. For the $500^{\circ} \mathrm{C}$ annealed sample, the temperature is too high which breaks the film apart exposing glass surface underneath the films and forms $\mathrm{MoO}_{3}$ islands.

\subsection{EXPERIMENTAL METHODS}

Chemicals and Reagents: Microscope glass slides were purchased from Sigma-Aldrich. Polystyrene microspheres were purchased from Thermo Scientific. Molybdenum Trioxide $\left(\mathrm{MoO}_{3}\right)$ e-beam evaporator pallets were purchased from Kurt J. Lesker. Acetone, methanol, sulphuric aicd and Hydrogen peroxide were purchased from Alfa Aesar. Deionized (D.I) water was purchased from Millipore Corp. Nitrogen (ultra high purity 99.999\%) tank was purchased from Matheson Tri-gas.

Instrumentation and Characterization: E-beam evaporator (Temescal) was used to deposit Molybdenum trioxide thin films. Tube furnace (Lindberg/Blue M) was used to anneal the sample under Nitrogen atmosphere. Scanning Electron Microscopy (SEM) images were taken under a JEOL JSM-7600F with an accelerating voltage of 5kV. Shimadzu UV-2550 UV-Vis spectrometer equipped with an integrating sphere (UV 2401/2) was used to acquire the ultraviolet (UV)-Visible absorption spectra. X-ray Diffraction (XRD) measurements were performed with PANalytical X'pert Pro X-ray diffractometer to study the crystallinity of the samples. X-ray Photoelectron spectroscopy (XPS) measurements were performed using a Physical Electronics PHI 5000 VersaProbe X-Ray Photoelectron Spectrometer to analyze the chemical state and electronic

structure of samples. Hall effect measurements were also taken to estimate the resistivity and carrier concentration of the thin films.

\section{Fabrication of $\mathrm{MoO}_{\mathrm{x}}$ thin films and annealing:}


Initially, glass slides were cut into $1.5 \mathrm{~cm} \times 1.5 \mathrm{~cm}$ pieces to be used as substrates. The glass pieces were cleaned by ultrasonicating in acetone, methanol and D.I water for five minutes in each solution and dried using nitrogen gas. $300 \mathrm{~nm} \mathrm{MoO}_{3}$ thin film was evaporated on glass pieces using e-beam evaporator. As deposited $\mathrm{MoO}_{3}$ samples were then annealed in $\mathrm{N}_{2}$ atmosphere at $200^{\circ} \mathrm{C}$, $300^{\circ} \mathrm{C}, 400^{\circ} \mathrm{C}$ and $500^{\circ} \mathrm{C}$ for 3 hrs at ramp rate of $5^{\circ} \mathrm{C} / \mathrm{min}$ to from $\mathrm{MoO}_{3-\mathrm{x}}$.

\section{Finite-Difference Time-Domain (FDTD) Simulation}

Optical properties of $\mathrm{MoO}_{3-\mathrm{x}}$ films were analyzed by FDTD simulations. The software Lumerical FDTD Solutions is commercially available. A grid size of $5 \mathrm{~nm}$ was used to construct the simulation cells. A continuous plane wave from 200 to $1800 \mathrm{~nm}$ was used as the input light source. In modeling the dielectric function of $\mathrm{MoO}_{3-\mathrm{x}}$ films, a Drude Model was used. The Drude formula is given by

$$
\varepsilon(\omega)=1-\frac{\omega_{p}^{2}}{\omega^{2}+i \gamma \omega}
$$

In the above equation, $\omega_{p}$ is the plasma frequency calculated by $\omega_{p}=\sqrt{\frac{N e^{2}}{\varepsilon_{0} m_{e}}}$ where $\mathrm{N}$ is the free carrier density, $\varepsilon_{0}$ is the permittivity of free space and $m_{e}$ is the effective mass of the free carrier (electron in this case). The damping constant $\gamma$ can be calculated by $\gamma=\frac{N e^{2}}{\sigma m_{e}}$ where $\sigma$ is the conductivity. Since the $\mathrm{MoO}_{3-\mathrm{x}}$ film was modelled as a rough surface, its surface roughness (RMS, or root mean square) was fixed at $30 \mathrm{~nm}$, unless study of surface roughness on the plasmon resonance was conducted where the RMS was varied from 10 to $50 \mathrm{~nm}$. 


\subsection{RESULTS and DISCUSSIONS}

Optical Characterization of $\mathrm{MoO}_{3}$ thin films. The optical properties of $\mathrm{MoO}_{3}$ thin films were characterized by taking UV-Vis absorption spectra. Figure 6.2(a) shows the comparison of absorption spectra of $\mathrm{MoO}_{3}$ films annealed in $\mathrm{N}_{2}$ at different temperatures. The as-deposited $\mathrm{MoO}_{3}$ thin film has an absorption band edge around $420 \mathrm{~nm}$ and there is no photo absorption at longer Figure 6. 2:(a) UV-Vis spectra of MoO3 annealed at different temperatures in N2 (b) Optical image of MoO3 film on glass annealed at different temperature in N2

wavelengths. In contrast, the films annealed in $\mathrm{N}_{2}$ showed a clear visible light absorption. The absorption peak blue shifted from $800 \mathrm{~nm}$ to $600 \mathrm{~nm}$ with the increase in the annealing temperature from $200^{\circ} \mathrm{C}$ to $400^{\circ} \mathrm{C}$. This strong absorption is due to the Localized Surface Plasmon Resonance

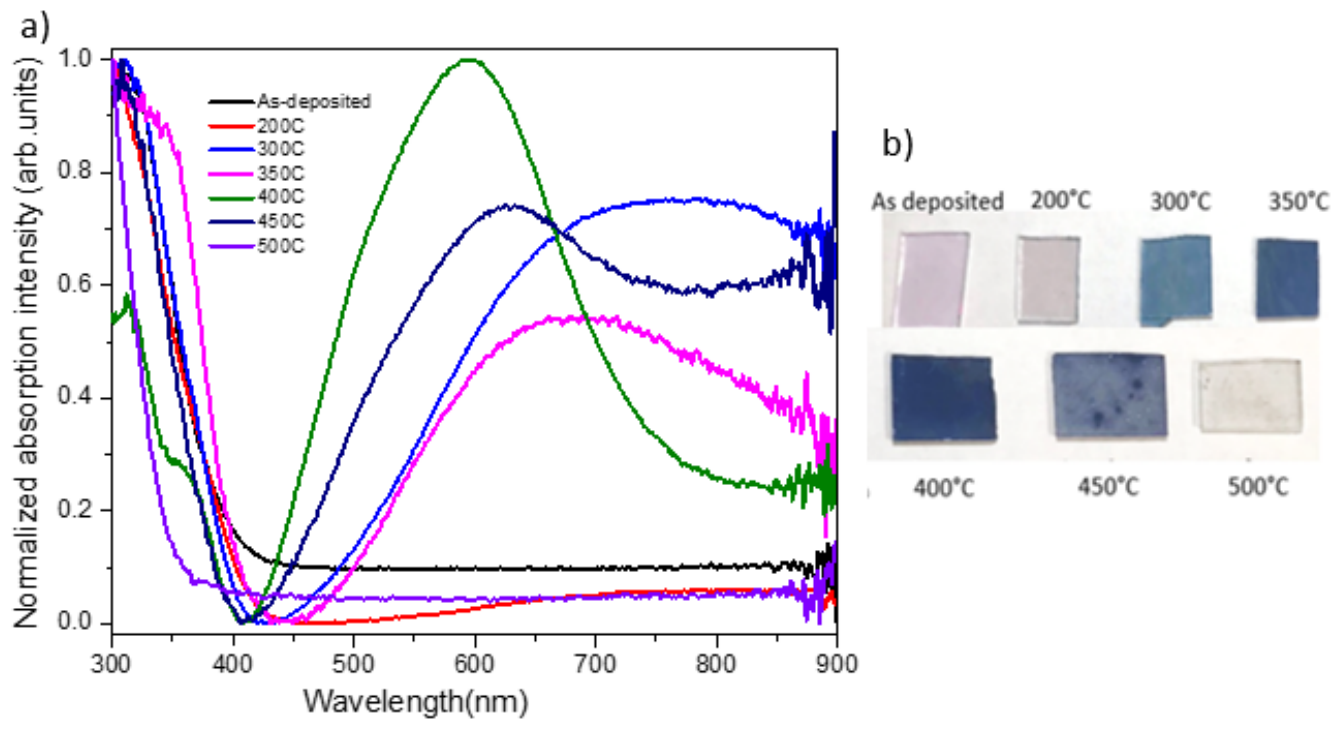

(LSPR) generated from the free carriers, induced during nitrogen annealing. During annealing process nitrogen reduces the molybdenum trioxide and induces oxygen vacancies leading to the increase in the carrier concentration. The bulk plasmon frequency is strongly dependent on the free carrier concentration of the material, which enabled broad range tunability of LSPR peak from NIR to Visible region.

$$
\omega_{p}=\sqrt{\frac{N q^{2}}{\varepsilon_{0} m_{e}}}
$$


$\omega_{p}$ is the bulk plasmon frequency, $\mathrm{N}$ is the free carrier density, $\varepsilon_{0}$ is the permittivity of free space and $m_{e}$ is the effective mass of the free carrier (electron in this case).

For the films annealed at lower temperature the plasmon peak is weak and present at longer wavelengths indicating less number of oxygen vacancies are created. When the annealing temperature is increased, the LSPR peak strengthened and blue shifted revealing more number of free carriers are generated. This phenomenon is confirmed by calculating the free carrier concentration from Hall effect measurement system. The as-deposited film is insulating which makes Hall system impossible to acquire carrier concentration and conductivity of the sample. $\mathrm{MoO}_{3}$ films which are annealed showed increase in carrier density and conductivity with increase in temperature up to $400^{\circ} \mathrm{C}$. The carrier concentration of the $\mathrm{MoO}_{3-\mathrm{X}}$ annealed at $400^{\circ} \mathrm{C}$ was determined to be $5^{*} 10^{20}$. The strongest LSPR is observed at around $600 \mathrm{~nm}$. Beyond $400^{\circ} \mathrm{C}$, in spite of increase in the temperature the plasmon strength decreased and shifted to longer wavelengths, due to collapsing of the film to form islands.

Figure 6.2(b) shows the optical image of the $\mathrm{MoO}_{3}$ films on glass substrate. The asdeposited sample is optically transparent. The annealed samples show blue color and the intensity gradually increased upto $400^{\circ} \mathrm{C}$ above which the blue tint disappeared. This phenomenon is correlated with the LSPR peak, explaining strong LSPR coexisted when the sample is deep blue in color. This phenomenon is due to the outer-d electrons intravalence charge transfer between $\mathrm{Mo}^{+5}$ and $\mathrm{Mo}^{+6} \cdot{ }^{20}$

\section{Tunable plasmon peak in $\mathrm{MoO}_{3-\mathrm{x}}$ films}

In metals, the conductivity and carrier density are fairly constant, allowing other parameters like shape, size and dielectric plays an important role in tuning the plasmon peak. In semiconductors, especially in this case the conductivity and carrier density of $\mathrm{MoO}_{3-\mathrm{x}}$ film changes with annealing

temperature making plasmon peak tunable over a large range. Thus, the important parameters like carrier density, conductivity and roughness effects on plasmon peak were analyzed. Initially a rough $\mathrm{MoO}_{3}$ film is modeled with surface roughness RMS 30, using Optiwave FDTD. Figure. $6.3(\mathrm{~b})$ 

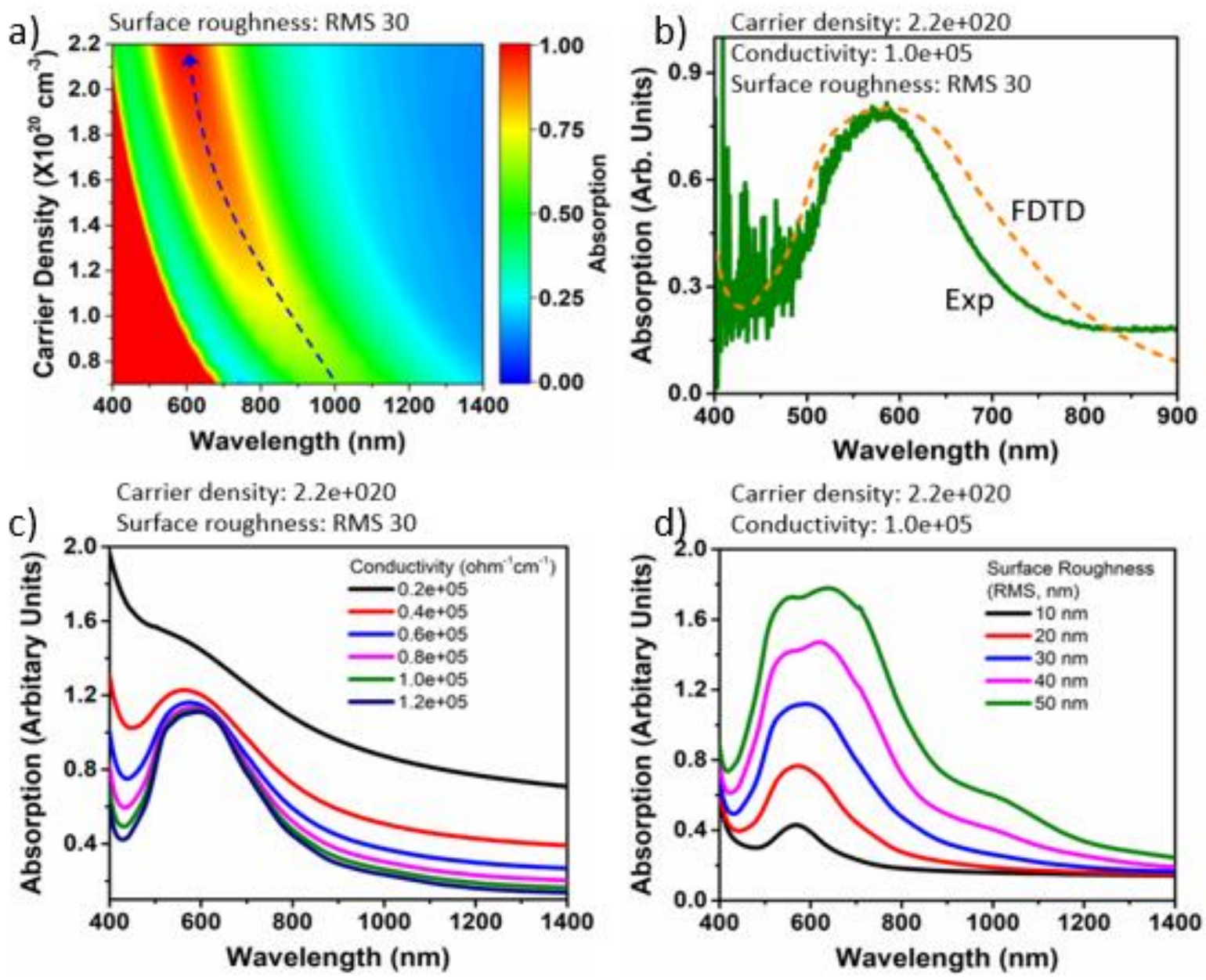

Figure 6. 3: a) Dependence of plasmon peak on carrier density at surface roughness RMS 30. $b$ ) Experimental (Exp) and FDTD-simulated (FDTD) absorption spectra of MoOx film c) plasmon peak strength dependence on conductivity of the film d) d) plasmon peak wavelength dependence on surface roughness

shows a good match of experimental and simulated absorption spectra where carrier density and conductivity of the $\mathrm{MoO}_{3-\mathrm{x}}$ considered to be $2.2 * 10^{20}$ and $1 * 10^{5}$ respectively. Further each parameter was analyzed to get an optimized plasmon peak. (Figure 6.3(a)) shows, as the carrier density increases the plasmon peak blue shifted which was discuss earlier. The other important parameter is the conductivity.

$$
\gamma=\frac{N e^{2}}{\sigma m_{e}}
$$

where $\sigma$ is the conductivity and $\gamma$ is damping constant. 
The plasmon damping increases with the decrease in conductivity from the above equation. The plasmon resonance is strong for highly conductive materials and collapses for low conductive or insulating materials. This is one of the reasons for as-deposited $\mathrm{MoO}_{3-\mathrm{x}}$ does not have a plasmon peak. This also explains, why there is no plasmon resonance for all degenerate semiconductors. Further, the factor of roughness is analyzed. A localized plasmon resonance exists only when there are nanoparticles. The smooth $\mathrm{MoO}_{3-\mathrm{x}}$ films cannot support LSPR, Figure 6.1(a) shows asdeposited $\mathrm{MoO}_{3-\mathrm{x}}$ films where the surface roughness is negligible. However, with increase in temperature the surface roughness increases allowing plasmon to strengthen. This patter is in well agreement with simulations shown in Figure 6.2(d). Thus all the parameters are tuned precisely to allow the plasmon resonance to fall in visible region.

X-ray Diffraction (XRD) (Figure 6.4) was performed to study the crystallinity and chemical state of the $\mathrm{MoO}_{3}$ samples. From the XRD spectra in Figure 6.4, the as-deposited $\mathrm{MoO}_{\mathrm{x}}$ sample shows an amorphous nature with no defined peaks. After heat treatment at $300{ }^{\circ} \mathrm{C}$ under $\mathrm{N}_{2}$ where the LSPR peak begins to appear, the $\mathrm{MoO}_{\mathrm{x}}$ sample still retains an amorphous nature with no defined peaks. Once the heat treatment reaches $400{ }^{\circ} \mathrm{C}$ under $\mathrm{N}_{2}$, the $\mathrm{MoO}_{\mathrm{x}}$ sample distinct crystalline peaks attributable to a dominant $\mathrm{Mo}_{5} \mathrm{O}_{14}$ (ICSD 98-007-2639) phase with a very minor $\mathrm{MoO}_{2}$ phase (ICSD 98-003-6263). Further heating at $500{ }^{\circ} \mathrm{C}$ under $\mathrm{N}_{2}$ results in a near complete conversion of the sample to $\mathrm{MoO}_{2}$. 


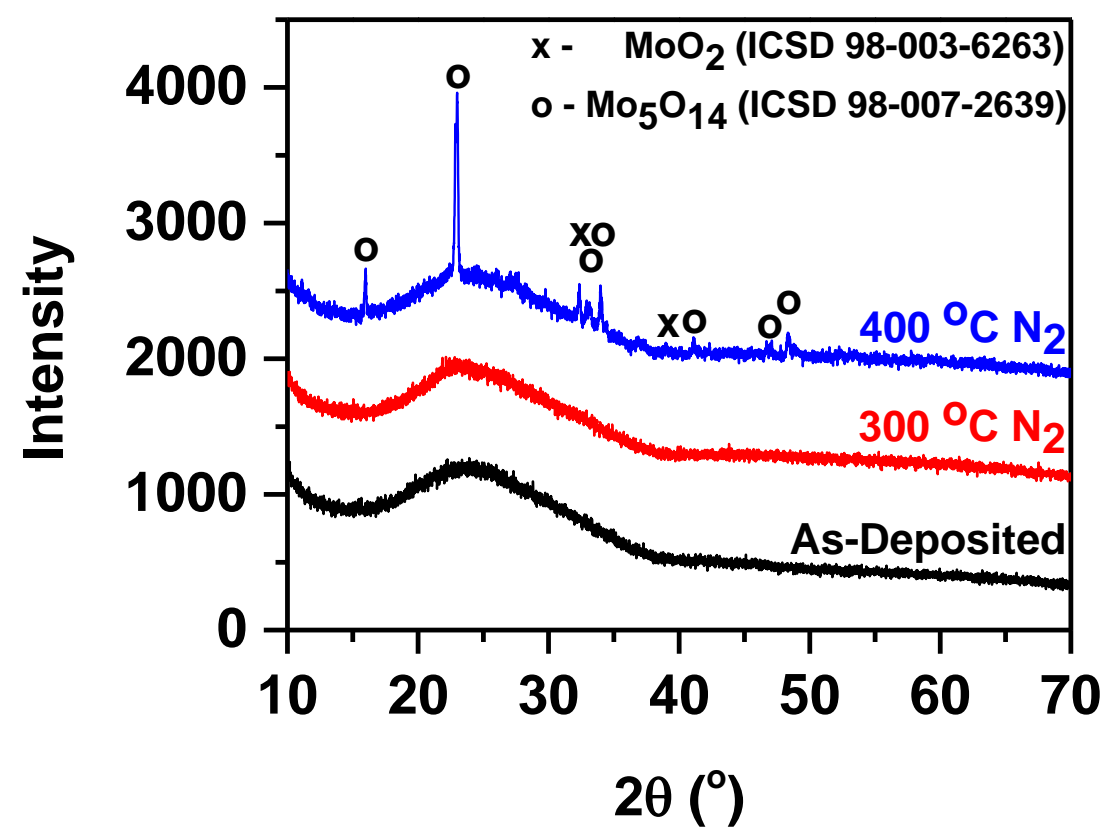

Figure 6. 4: XRD spectra for MoOx thin films on glass substrates after different heat treatment temperatures under $N_{2}$.

To determine the effects of the heat treatments under $\mathrm{N}_{2}$ on the chemical state of the $\mathrm{MoO}_{3}$ films, core level (Figure 6.5) X-ray Photoelectron spectroscopy (XPS) was performed. The as-deposited $\mathrm{MoO}_{3}$ samples shows only one peak at $233.0 \mathrm{eV}$ corresponding to $\mathrm{Mo}^{6+}$ states ${ }^{21}$. Upon heating at $300{ }^{\circ} \mathrm{C}$ under $\mathrm{N}_{2}$, the $\mathrm{MoO}_{x}$ sample show peaks at $232.8 \mathrm{eV}$ and $231.8 \mathrm{eV}$ corresponding to $\mathrm{Mo}^{6+}$ and $\mathrm{Mo}^{5+}$ states respectively. ${ }^{21,22}$ In addition, an additional peak in the $\mathrm{O} 1 \mathrm{~s}$ spectrum at $531.6 \mathrm{eV}$ appears that is associated with the chemical environment of oxygen anions near oxygen vacancies within metal oxides. ${ }^{23}$ The emergence of the new $\mathrm{Mo}^{5+}$ peak in the Mo3d spectrum and new $\mathrm{O} 1 \mathrm{~s}$ peak indicate that the heat treatment under $\mathrm{N}_{2}$ results in the formation of oxygen vacancies and reduction of $\mathrm{Mo}^{6+}$ to $\mathrm{Mo}^{5+}$ to maintain overall charge neutrality of the sample. Further heating at $400{ }^{\circ} \mathrm{C}$ under $\mathrm{N}_{2}$ results in further reduction of the sample evident from the formation of a new peak at $230.0 \mathrm{eV}$ corresponding to $\mathrm{Mo}^{4+}$ states and an increase in the relative peak intensity of the O1s peak due to the presence of oxygen vacancies $(531.8 \mathrm{eV})$. The mixed $\mathrm{Mo}^{4+} / \mathrm{Mo}^{5+} / \mathrm{Mo}^{6+}$ state of the sample heated at $400{ }^{\circ} \mathrm{C}$ under $\mathrm{N}_{2}$ is consistent with the dominant $\mathrm{Mos}_{5} \mathrm{O}_{14}$ crystal phase of the sample. 


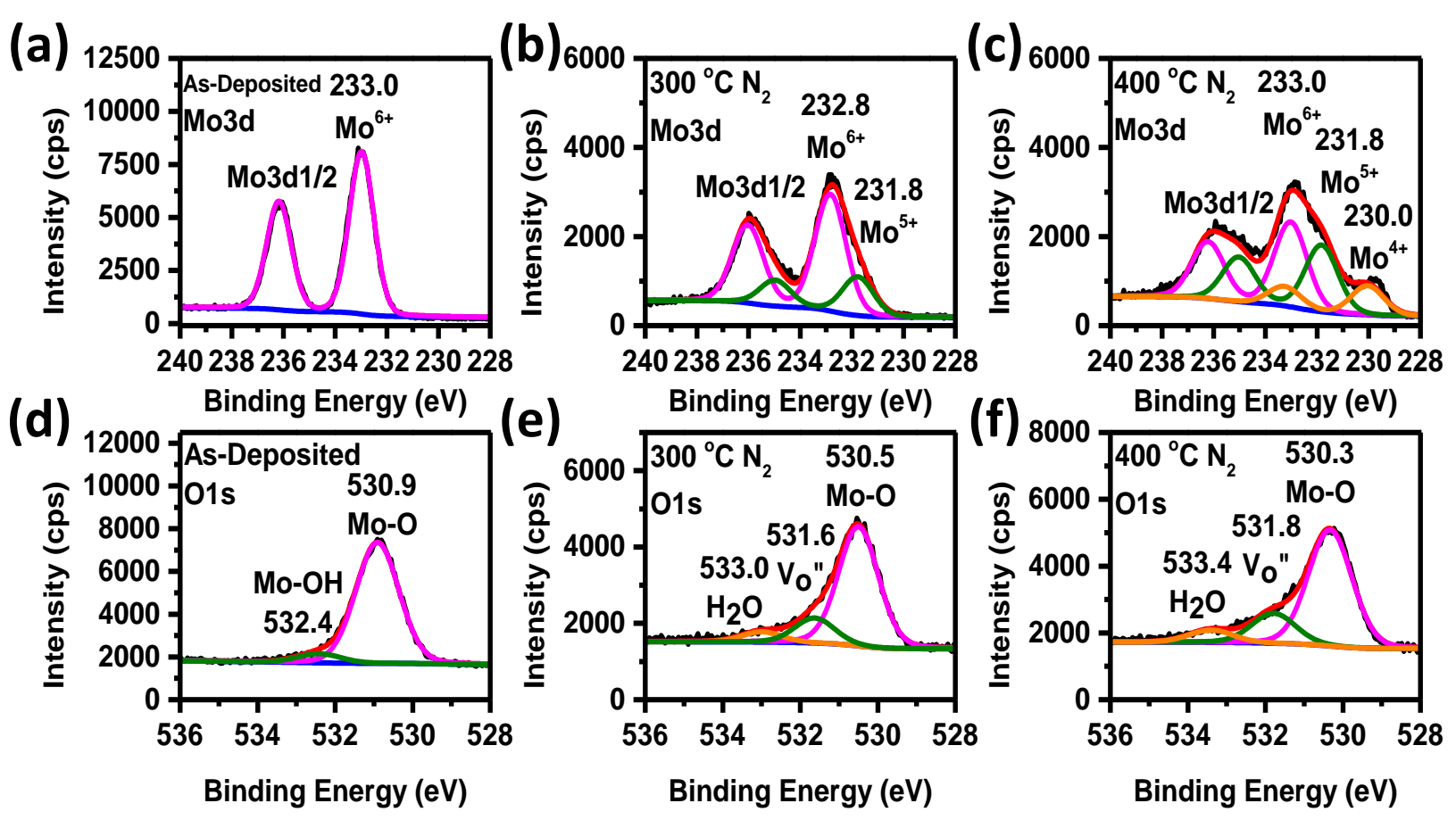

Figure 6. 5: XPS core level spectra for Mo3d (a-c) and Ols (d-f) of MoOx films on glass; $(a, d)$ As-deposited MoOx, $(b, e)$ MoOx film heat treated at $300{ }^{\circ} \mathrm{C}$ under N2, $(c, f)$ MoOx film heat treated at $400{ }^{\circ} \mathrm{C}$ under $\mathrm{N}_{2}$

To determine the effects of the $\mathrm{Mo}^{4+}, \mathrm{Mo}^{5+}$, and oxygen vacancy states on the electronic structure within the bandgap of the $\mathrm{MoO}_{\mathrm{x}}$ samples, valence band XPS spectra (Figure 6.6) were measured.

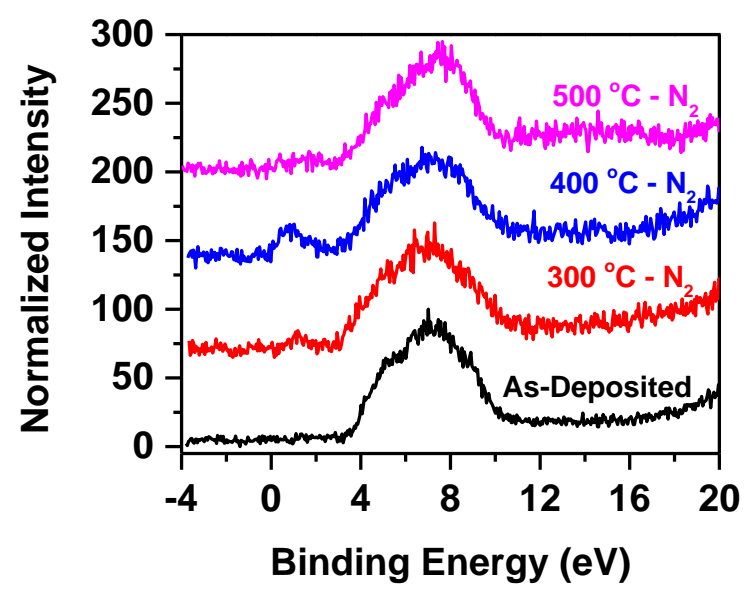

Figure 6. 6:Normalized XPS valence band spectra for MoOx films under different heat treatment temperatures under $\mathrm{N}_{2}$. 
As can be seen in (Figure 6.6), the as-deposited $\mathrm{MoO}_{3}$ samples have a clear valence band edge with no mid-gap states within the bandgap. Upon heating at $300{ }^{\circ} \mathrm{C}$ under $\mathrm{N}_{2}$, mid-gap electronic states begin to appear within the bandgap with a peak energy $1.9 \mathrm{eV}$ above the valence band edge. Further heating at $400{ }^{\circ} \mathrm{C}$ under $\mathrm{N}_{2}$ results in formation of additional mid-gap states with energies up to the Fermi level of the sample (Binding Energy $=0$ ). This indicates that the occupied mid-gap states from $\mathrm{Mo}^{4+} / \mathrm{Mo}^{5+}$ and oxygen vacancy states are the source of free carriers that improve the electronic conductivity and results in the plasmon resonance of the $\mathrm{MoO}_{\mathrm{x}}$ samples heated at $300{ }^{\circ} \mathrm{C}$ and $400{ }^{\circ} \mathrm{C}$ under $\mathrm{N}_{2}$.

However, upon heating at $500{ }^{\circ} \mathrm{C}$ under $\mathrm{N}_{2}$, the mid-gap states that were present with the $\mathrm{MoO}_{\mathrm{x}}$ samples heated at $300{ }^{\circ} \mathrm{C}$ and $400{ }^{\circ} \mathrm{C}$ under $\mathrm{N}_{2}$ disappear. The disappearance of the midgap states upon heating at $500{ }^{\circ} \mathrm{C}$ under $\mathrm{N}_{2}$ is consistent with the disappearance of the plasmon peak in the UV-Vis absorbance spectrum. In addition to the mid-gap states disappearing, there is a significant decrease in surface $\mathrm{Mo}^{4+}$ and $\mathrm{Mo}^{5+}$ states in the core-level XPS spectrum with the $\mathrm{MoO}_{\mathrm{x}}$ sample heated at $500{ }^{\circ} \mathrm{C}$ under $\mathrm{N}_{2}$ despite the near total conversion of the sample to $\mathrm{MoO}_{2}$. The crystal phase transformation and morphology changes the overall electronic structure and removes the plasmonic nature of the sample.

\subsection{CONCLUSION}

In summary plasmonic $\mathrm{MoO}_{3-x}$ films were fabricated, by inducing oxygen vacancies. The samples exhibit plasmon which is stable and repeatable. The LSPR peak is tunable over the large range from $600 \mathrm{~nm}$ to $1000 \mathrm{~nm}$. Chip based fabrication is followed for systematic study of plasmon intensity by varying the experimental parameters. The parameters which effect the plasmon resonance were tuned controllably and compared with the simulated results, which shows a perfect match. The analysis of the visible plasmon resonance in chip based semiconductor substrates offers promising applications in plasmonics. 


\section{References}

[1] Haes AJ, Van Duyne RP. A Nanoscale Optical Biosensor: Sensitivity and Selectivity of an Approach Based on the Localized Surface Plasmon Resonance Spectroscopy of Triangular Silver Nanoparticles. J Am Chem Soc 2002, 124:10596-10604.

[2] S. D. Hudson and G. Chumanov. Bioanalytical applications of SERS (surface-enhanced Raman spectroscopy. Anal Bioanal Chem. 2009 Jun;394(3):679-86

[3] Xuefei gao et.al, Paper-Based Surface-Enhanced Raman Scattering Lateral Flow Strip for Detection of Neuron-Specific Enolase in Blood Plasma Anal. Chem., 2017, 89 (18), pp 1010410110

[4] P. Zheng et al, Detection of nitrite with a surface-enhanced Raman scattering sensor based on silver nanopyramid array, Analytica Chimica Acta 1040 (2018) 158165.

[5] Curto, A.G Volpe, G,Taminiau, T.H. Kreuzer, M.P Quidant, van Hulst, N. F. Unidirectional Emission of a Quantum Dot Coupled to a Nanoantenna. Science 2010, 329, 930-933, DOI: 10.1126/science.1191922.

[6] Peng Zheng, Sujan Kasani and Nianqiang Wu, Converting plasmonic light scattering to confined light absorption and creating plexcitons by coupling a gold nano-pyramid array onto a silica-gold filmNanoscale Horiz., 2019, 4, 516-525.

[7] Scott K. Cushing and Nianqiang Wu, Plasmon-Enhanced Solar Energy Harvesting, Electrochem. Soc. Interface Summer 2013 volume 22, issue 2, 63-67

[8] Yang Liu, Maixian Liu and Mark T. Swihart. Plasmonic Copper Sulfide-Based Materials: A Brief Introduction to Their Synthesis, Doping, Alloying, and Applications J. Phys. Chem. C 2017, 121, 13435-13447.

[9] Luther JM, Jain PK, Ewers T, Alivisatos AP. Localized surface plasmon resonances arising from free carriers in doped quantum dots, Nat Mater. 2011 May;10(5):361-6

[10] Dirk Dorfs, Thomas Hartling, Karol Miszta et al. Reversible tunability of the near-infrared valence band plasmon resonance in $\mathrm{Cu}(2-\mathrm{x}) \mathrm{Se}$ nanocrystals. J Am Chem Soc. $2011 \mathrm{Jul}$ 27;133(29):11175-80

[11] Seung Hyuk Lee, Hiroyasu Nishi and Tetsu Ttsuma. Tunable plasmon resonance of molybdenum oxide nanoparticles synthesized in non-aqueous media Chem. Commun., 2017,53, 12680. 
[12] Karthish Manthiram and A. Paul Alivisatos. Tunable Localized Surface Plasmon Resonances in Tungsten Oxide Nanocrystals J. Am. Chem. Soc. 2012, 134, 3995-3998.

[13] Manal M.Y.A.Alsaif, Kay Latham et al. Tunable Plasmon Resonances in Two-Dimensional Molybdenum Oxide Nano flakes. Adv. Mater. 2014, 26, 3931-3937.

[14] Xianjun Tan, Lingzhi Wang et al. Plasmonic MoO33-x @ MoO3 nanosheets for highly sensitive SERS detection through nanoshell-isolated electromagnetic enhancement Chem. Commun., 2016, 52, 2893.

[15] Manal M.Y.A.Alsaif, Kay Latham et al. Tunable Plasmon Resonances in Two-Dimensional Molybdenum Oxide Nano flakes. Adv. Mater. 2014, 26, 3931-3937.

[16] Gorbachev, V. V.; Putilin, I. M, Some parameters of band structure in copper selenide and telluride. Phys. Status Solidi A 1973, 16, 2, 553-559.

[17] Cong S, Yuan Y, Chen Z, Hou J, Yang M, Su Y, Zhang Y, Li L, Li Q, Geng F, Zhao Z, Noble metal-comparable SERS enhancement from semiconducting metal oxides by making oxygen vacancies, Nat Commun. 2015 Jul 17;6:7800.

[18] Wei Huang, Yan Zhou, Jiayan Du,Yuequan Deng, and Yi He, Versatile Visual Logic Operations Based on Plasmonic Switching in Label-Free Molybdenum Oxide Nanomaterials, Anal. Chem. 2018, 90, 2384-2388.

[19] Haibo Yin,a Yasutaka Kuwahara,ab Kohsuke Mori,abc Hefeng Cheng,a Meicheng Wena and Hiromi Yamashita, High-surface-area plasmonic $\mathrm{MoO}_{3-\mathrm{x}}$ : rational synthesis and enhanced ammonia borane dehydrogenation activity, J. Mater. Chem. A, 2017, 5, 8946

[20] Q. Huang, S. Hu, J. Zhuang and X. Wang. MoO3-X-Based Hybrids with Tunable Localized Surface Plasmon Resonances: Chemical Oxidation Driving Transformation from Ultrathin Nanosheets to Nanotubes. Chem. - Eur. J., 2012, 18, 15283.

[21] Özkar S, Ozin, G. A, Prokopowicz R. A. Photoxidation of hexacarbonylmolybdenum (0) in sodium zeolite Y to yield redox-interconvertible molybdenum (VI) oxide and molybdenum (IV) oxide monomers. Chem. Mater.1992461380-1388.

[22] Anwar M, Hogarth C. A, Bulpett R. Effect of substrate temperature and film thickness on the surface structure of some thin amorphous films of $\mathrm{MoO}_{3}$ studied by $\mathrm{x}$-ray photoelectron spectroscopy (ESCA). J. Mater. Sci., 1989, 24 (9), 3087-3090.

[23] Kim. J. H, Jang Y. J, Kim J. H, Jang J.-W, Choi S. H, Lee J.-S. Defective ZnFe $2 \mathrm{O}_{4}$ nanorods with oxygen vacancy for photoelectrochemical water splitting. Nanoscale, 2015, 7, 19144-19151. 
[24] H. Cheng, X. Qian, Y. Kuwahara, K. Mori, H. Yamashita. A Plasmonic Molybdenum Oxide Hybrid with Reversible Tunability for Visible-Light-Enhanced Catalytic Reactions. Adv. Mater. 2015, 27, 4616-4621.

[25] H. Cheng, T. Kamegawa, K. Mori, H. Yamashita, Angew. Chem. Int. Ed. 2014, 53, $2910-$ 2914; Angew. Chem. 2014, 126, 2954-2958

[26] Ilka Kriegel, Francesco Scotognella, Liberato Manna, Plasmonic Doped Semiconductor Nanocrystals: Properties, Fabrication, Applications and Perspectives, arXiv:1701.05972 [condmat.mtrl-sci]

[27] Alberto Comin and Liberato Manna, New materials for tunable plasmonic colloidal nanocrystals, Chem. Soc. Rev., 2014, 43, 3957

[28] Wang, X.; Ke, Y.; Pan, H.; Ma, K.; Xiao, Q.; Yin, D.; Wu, G.; Swihart, M. T. Cu-Deficient Plasmonic Cu2-xS Nanoplate Electrocatalysts for Oxygen Reduction. ACS Catal. 2015, 5, 2534-2540.

[29] Chuanhui Zhu, Qun Xu, Liang Ji, Yumei Ren, and Mingming Fang, Room-temperature Synthesis of Amorphous Molybdenum Oxide Nanodots with Tunable Localized Surface Plasmon Resonances, Chem. Asian J. 2017, 12, 2980 - 2984

[30] Ning Li, Yamei Li, Guangyao Sun, Yijie Zhou, Shidong Ji, Heliang Yao, Xun Cao, Shanhu Bao and Ping Jin, Enhanced photochromic modulation efficiency: a novel plasmonic molybdenum oxide hybrid, Nanoscale, 2017, 9, 8298

[31] Naik, G.; Boltasseva, A. Plasmonics and Metamaterials: Looking beyond Gold and Silver. SPIE Newsroom, 2012.10.1117/ 2.1201201.004077

[32] Jacob B. Khurgin, Replacing noble metals with alternative materials in plasmonics and metamaterials: how good an idea?, Phil. Trans. R. Soc. A 375: 20160068.

[33] Urcan Guler, Alexander V. Kildishev, Alexandra Boltasseva and Vladimir M. Shalaev, Plasmonics on the slope of enlightenment: the role of transition metal nitrides, Faraday Discuss., $2015,178,71$

[34] P.Patsalas N.Kalfagiannis S.Kassavetis G.Abadias D.V.Bellas Ch.Lekka E.Lidorikis, Conductive nitrides: Growth principles, optical and electronic properties, and their perspectives in photonics and plasmonics, materials science and engineering R 123(2018) 1-55. 
[35] Adrien Lalisse ,Gilles Tessier, Jérome Plain, Guillaume Baffou, Plasmonic efficiencies of nanoparticles made of metal nitrides (TiN, $\mathrm{ZrN}$ ) compared with gold, Scientific Reports volume6, Article number: 38647 (2016)

[36] Adela Habib, Fred Florio and Ravishankar Sundararaman, Hot carrier dynamics in plasmonic transition metal nitrides, J. Opt. 20 (2018) 064001 (9pp)

[37] Jacob A. Faucheaux, Alexandria L. D. Stanton, and Prashant K. Jain, Plasmon Resonances of Semiconductor Nanocrystals: Physical Principles and New Opportunities, J. Phys. Chem. Lett. 2014, 5, 6, 976-985

[38] van der Stam, W.; Berends, A. C, de Mello Donega, C. Prospects of Colloidal Copper Chalcogenide Nanocrystals. ChemPhysChem 2016, 17, 559-581

[39] Hui Zhang, Runmin Zhang, Katelyn S. Schramke, Nicholas M. Bedford, Katharine Hunter, Uwe R. Kortshagen, and Peter Nordlander, Doped Silicon Nanocrystal Plasmonics [40] Rowe, D. J.; Jeong, J. S.; Mkhoyan, K. A.; Kortshagen, U. R. Phosphorus-Doped Silicon Nanocrystals Exhibiting Mid-Infrared Localized Surface Plasmon Resonance. Nano Lett. 2013, $13,1317-1322$

[41] Kramer, N. J.; Schramke, K. S.; Kortshagen, U. R. Plasmonic Properties of Silicon Nanocrystals Doped with Boron and Phosphorus. Nano Lett. 2015, 15, 5597-5603

[42] Kriegel, I.; Rodríguez-Fernandez, J.; Wisnet, A.; Zhang, H.; Waurisch, C.; Eychmüller, A.; Dubavik, A.; Govorov, A. O.; Feldmann, J. Shedding Light on Vacancy-Doped Copper Chalcogenides: ShapeControlled Synthesis, Optical Properties, and Modeling of Copper Telluride Nanocrystals with near-Infrared Plasmon Resonances. ACS Nano 2013, 7, 4367-4377.

[43] Yang Liu, Maixian Liu, and Mark T. Swihart, Plasmonic Copper Sulfide-Based Materials: A Brief Introduction to Their Synthesis, Doping, Alloying, and Applications, J. Phys. Chem. C 2017, 121, 13435-13447

[44] Bao Yue Zhang et.al Degenerately Hydrogen Doped Molybdenum Oxide Nanodisks for Ultrasensitive Plasmonic Biosensing, Adv. Funct. Mater. 2018, 28, 1706006 [45] Zhou, S.; Pi, X.; Ni, Z.; Ding, Y.; Jiang, Y.; Jin, C.; Delerue, C, Yang, D.; Nozaki, T. Comparative Study on the Localized Surface Plasmon Resonance of Boron- and PhosphorusDoped Silicon Nanocrystals. ACS Nano 2015, 9, 378-386. 
[46] Hui Zhang,Runmin Zhang,Katelyn S. Schramke,Nicholas M. Bedford, Katharine Hunter, Uwe R. Kortshagen, and Peter Nordlander, Doped Silicon Nanocrystal Plasmonics, ACS Photonics 2017, 4, 963-970

[47] Abhitosh Kedia ${ }^{a}$ and Pandian Senthil Kumar, Controlled reshaping and plasmon tuning mechanism of gold nanostars, J. Mater. Chem. C, 2013,1, 4540-4549

[48] Stephan Link, and Mostafa A. El-Sayed, Size and Temperature Dependence of the Plasmon Absorption of Colloidal Gold Nanoparticles, J. Phys. Chem. B 1999, 103, 21, 4212-4217

[49] Sujan Kasani, Peng Zheng, and Nianqiang Wu, Tailoring Optical Properties of a Large-Area Plasmonic Gold Nanoring Array Pattern, J. Phys. Chem. C 2018, 122, 13443-13449

[50] Jack J. Mock, David R. Smith, and Sheldon Schultz, Local Refractive Index Dependence of Plasmon Resonance Spectra from Individual Nanoparticles, Nano Letters 2003, 3, 4, 485-491

[51] Kelly, K. L; Coronado, E.; Zhao, L. L.; Schatz, G. C. The Optical Properties of Metal Nanoparticles: The Influence of Size, Shape, and Dielectric Environment J. Phys. Chem. B 2003, 107, 668- 677

[52] Erwin, S. C.; Zu, L.; Haftel, M. I.; Efros, A. L.; Kennedy, T. A.; Norris, D. J. Doping Semiconductor Nanocrystals. Nature 2005, 436, 91-94.

[53] Guillermo Garcia, Raffaella Buonsanti, Evan L. Runnerstrom, Rueben J. Mendelsberg, Anna Llordes, Andre Anders, Thomas J. Richardson, and Delia J. Milliron, Dynamically Modulating the Surface Plasmon Resonance of Doped Semiconductor Nanocrystals, Nano Lett. 2011, 11, 44154420

[54] Edward Sachet, Mark D. Losego, Joshua Guske, Stefan Franzen, and Jon-Paul Maria, Midinfrared surface plasmon resonance in zinc oxide semiconductor thin films, Appl. Phys. Lett. 102, 051111 (2013)

[55] Dewangan, K.; Sinha, N. N.; Sharma, P. K.; Pandey, A. C.; Munichandraiah, N.; Gajbhiye, N. S. Synthesis and characterization of single-crystalline $\alpha-\mathrm{MoO} 3$ nanofibers for enhanced Li-ion intercalation applications. CrystEngComm 2011, 13, 927-933

[56] Li, Z. C.; Li, Y.; Zhan, E. S.; Ta, N.; Shen, W. J. Morphologycontrolled synthesis of $\alpha-\mathrm{MoO} 3$ nanomaterials for ethanol oxidation. J. Mater. Chem. A 2013, 1, 15370-15376.

[57] Kenji Adachi, Activation of plasmons and polarons in solar control cesium tungsten bronze and reduced tungsten oxide nanoparticles, J. Mater. Res., Vol. 27, No. 6, Mar 28, 2012. 
[58] Murray, C. B.; Norris, D. J.; Bawendi, M. G. Synthesis and Characterization of Nearly Monodisperse CdE (E = Sulfur, Selenium, Tellurium) Semiconductor Nanocrystallites. J. Am. Chem. Soc. 1993, 115, 8706-8715.

[59] Yixin Zhao, Hongcheng Pan, Yongbing Lou, Xiaofeng Qiu, JunJie Zhu and Clemens Burda, Plasmonic $\mathrm{Cu}_{2-\mathrm{x}} \mathrm{S}$ Nanocrystals: Optical and Structural Properties of Copper-Deficient Copper(I) Sulfides, J. Am. Chem. Soc., 2009, 131 (12), pp 4253-4261

[60] M. Balkan, A. Aziza, and E. Amzallag, Infrared Absorption in Heavily Doped n-Type Si Phys. Status Solidi 31, 323 (1969).

[61] James C. Ginn, Robert L. Jarecki, Eric A. Shaner, and Paul S. Davids, Infrared plasmons on heavily-doped silicon, J. Appl. Phys. 110, 043110 (2011)

[62] Liang, X.; Zhang, X. J.; You, T. T.; Wang, G. S.; Yin, P. G.; Guo, L. Controlled assembly of one-dimensional MoO3@Au hybrid, CrystEngComm, 2016,18, 7805-7813

[63] Xu, Y.; Zhou, M.; Wang, X.; Wang, C. L.; Liang, L. Y.; Grote, F.; Wu, M. H.; Mi, Y.; Lei, Y. Enhancement of Sodium Ion Battery Performance Enabled by Oxygen Vacancies. Angew. Chem., Int. Ed. 2015, 54, 8768-8771

[64] Song, Y. H.; Zhao, J. H.; Zhao, Y.; Huang, Z. F.; Li, Y. W.; Wu, G. J. Synthesis of $\alpha-M o O 3$ nanobelts with preferred orientation and good photochromic performance. CrystEngComm 2016, $18,6502-6512$.

[65] B. Tian, X. Liu, B. Tu, C. Yu, J. Fan, L. Wang, S. Xie, G. D. Stucky and D. Zhao, Selfadjusted synthesis of ordered stable mesoporous minerals by acid-base pairs Nat. Mater, 2003, 2,159

[66] Y. Li, W. Luo, N. Qin, J. Dong, J. Wei, W. Li, S. Feng, J. Chen, J. Xu, A. A. Elzatahry, M. H. Es-Saheb, Y. Deng and D. Zhao, Highly ordered mesoporous tungsten oxides with a large pore size and crystalline framework for H2S sensing Angew. Chem., Int. Ed., 2014, 53, 9035.

[67] Huang, Q., Hu, S., Zhuang, J., Wang, X., MoO3-x-Based Hybrids with Tunable Localized Surface Plasmon Resonances: Chemical Oxidation Driving Transformation from Ultrathin Nanosheets to Nanotubes Chem. Eur. J. 2012, 18, 15283.

[68] Yao, D. D., Ou, J. Z., Latham, K., Zhuiykov, S., O'Mullane, A. P., Kalantar-zadeh, K., Electrodeposited $\alpha$ - and $\beta$-Phase $\mathrm{MoO}_{3}$ Films and Investigation of Their Gasochromic Properties Cryst. Growth Des. 2012, 12, 1865-1870 
[69] Khademi, A.; Azimirad, R.; Zavarian, A. A.; Moshfegh, A. Z. Growth and Field Emission Study of Molybdenum Oxide Nanostars. J. Phys. Chem. C 2009, 113, 19298-19304

[70] Haoqi Ren, Shaodong Sun,Jie Cui, and Xifei Li, Synthesis, Functional Modifications, and Diversified Applications of

Molybdenum Oxides Micro-/Nanocrystals: A Review, Cryst. Growth Des. 2018, 18, 6326-6369

[71] Greenblatt, M., Molybdenum oxide bronzes with quasi-low-dimensional properties Chem. Rev. 1988, 88, 31- 53

[72] Deb S. K, Opportunities and challenges of electrochromic phenomena in transition metal oxides Sol. Energy Mater. Sol. Cells 1992, 25, 327.

[73] Goodenough, J. B, Metallic oxides, Prog. Solid State Chem. 1971, 5, 145 


\section{CHAPTER 7: SUMMARY AND FUTURE DIRECTION}

\subsection{SUMMARY}

In this dissertation, a reliable long range fabrication route is used for the fabrication of ordered plasmonic nanostructures. Using Nano Sphere Lithography nano ring array, nanopyramid array and coupled nanopyramid with gold film structures were fabricated and analyzed their optical properties. In this study, evolving of plasmonic mode in an individual structure and the properties of plasmonic coupling with different nano structures were analyzed. After understanding the fundamental properties of different plasmon resonance that can be excited in nanostructures, sensors were designed based on Surface Enhanced Raman Scattering (SERS) and Plasmon Enhanced Fluorescence (PEF) techniques. Using the local EM field enhancement, and fundamentally understanding the SERS and PEF enhancement criteria, the nanostructures were optimized for maximum enhancement. Innovation in the area of fabrication in plasmonics has direct impact on the field of energy harvesting. In this study a 3D nanostructure of nanopyramid array coupled to gold film was fabricated with a silica layer in between forming a plasmon gap mode. The structure traps light into the spacer layer exhibiting total light absorption.

In addition to exploiting noble metals such as gold and silver to study surface-enhanced spectroscopy, alternative cost-effective plasmonic materials have also been explored. In this dissertation, $\mathrm{MoO}_{3}$ semiconductor NC's were studied for plasmonic behavior and observed wide plasmon tunable range. The carrier concentration, physical dimension and conductivity parameters of $\mathrm{MoO}_{3}$ dependency on plasmon peak position is investigated. The results strongly indicate semiconductor NC's are highly promising alternative and inexpensive plasmonic materials.

\subsection{FUTURE DIRECTION}

The availability of fabrication techniques as well as the discovery and understanding of plasmonic modes are the key to plasmonic research success. In the past two decades, many fabrication methods have been developed to overcome the limitation of conventional photolithography. However, significant challenges remain for large scale fabrication of nanoarrays at nanoscale-resolution and low-cost with excellent repeatability, controllability and great flexibility in tuning optical properties. In many cases, simulation shows that hierarchical structures 
could exhibit extraordinary optical properties; however, no one fabrication method is available to make real structures. In particular, there are still technical barriers for fabrication of large-area, long-range-ordered periodic nano-array patterns. On the other hand, when plasmonic nano-arrays are used in practical application such as photovoltaics, photoelectrochemical cells (PECs), sensors and optoelectronics, plasmonic metal-semiconductor heterojunctions are required to enable plasmonic coupling between metals and semiconductors. This puts a constraint on the development of fabrication route. 


\section{Appendix}

\section{Publications}

[1] Tailoring Optical Properties of a Large-Area Plasmonic Gold Nano-ring Array Pattern, Sujan Kasani, P.Zheng and Nianqiang Wu; J.phys.Chem.C, 2017 (invited paper).

[2] Converting Plasmonic Light Scattering to Confined Light Absorption by coupling a Nanopyramid Array to a Silica-Gold Film Peng Zheng (co-first author), Sujan Kasani (co-first author) and Nianqiang Wu, Nanoscale Horizon, 2019, 516-525.

[3] A review of 2D and 3D plasmonic Nanostricture Array Patterns: Fabrication, Light management and Sensing Applications. Sujan Kasani, Kathrine Curtin and Nianqiang Wu, Nanophotonics (Invited, under review)

[4] Paper-Based Surface-Enhanced Raman Scattering Lateral Flow Strip for Detection of Neuron-Specific Enolase in Blood Plasma, Xuefei Gao,Peng Zheng, Sujan Kasani and Nianqiang Wu; Anal.Chem. 2017, 10104-10110.

[5] Origin of strong and narrow localized surface plasmon resonance of copper nanocubes, P.Zheng, H.Tang, S. Kasani, L. Huang, N.Q. Wu, Nano Research, 2018, 12, 63-68.

[6] Metal-organic framework coated titanium dioxide nanorod array p-n junction photoanode for solar water splitting, H yang, J Bright, Sujan Kasani and Nianqiang Wu, Nano Research, 2019, 643-650.

[7] Detection of Nitrite with a SERS Sensor Based on Silver Nanopyramid Array, Peng Zheng, Sujan Kasani, Habin Tang and Nianqiang Wu; Analytica Chimica Acta,2018, 158-165.

\section{Manuscripts (To be published)}

[1] Tunable Visible Light Plasmon Resonances of Molybdenum Oxide ( $\left.\mathrm{MoO}_{3-\mathrm{X}}\right)$ Thin Films. Sujan Kasani, Joeseph Bright, Peng Zheng and Nianqiang Wu.

[2] Enhanced Fluorescence of Metal- Organic Framework in Plasmonic Gap Mode. Sujan Kasani, Kathrine Curtin, Peng Zheng, Hui Yang and Nianqiang Wu.

[3] Near-Infrared Fluorescent Detection of Glial Fibrillary Acidic Protein in Blood Plasma Based on Gold Nano-pyramid Array. P.Zheng, Sujan Kasani and Nianqiang Wu 
[4] Detection of microRNA in Blood Plasma with Near-Infrared Fluorescent Nanoparticles on a Lateral Flow Test Strip, Xuefei Gao, Sujan Kasani and Nianqiang Wu

[5] Incorporation of Plasma Separation unit into Paper -based Lateral Flow Strip for Carcinoembryonic Antigen Detection in Whole Blood, Xuefei Gao, Sujan Kasani and Nianqiang $\mathrm{Wu}$

[6] Film-Coupled Copper Nanocube for Near-Infrared Fluorescence Enhancement Peng Zheng, Sujan Kasani, Nianqiang $\mathrm{Wu}$

[7] A Functionalized-UiO-66 MetalOrganic Framework Single Lithium-Ion Conductor. Hui Yang, Joeseph Bright, Sujan Kasani, Botong Liu and Nianqiang Wu. 